\title{
Development of an EMAT In-Line Inspection System For Detection, Discrimination, and Grading of Stress Corrosion Cracking In Pipelines
}

Final Report (Phase I)

Reporting Period Start Date: 10/1/2001

Reporting Period End Date: 9/30/2004

Principal Contributors:

Jeff Aron, Jeff Jia, Bruce Vance, Wen Chang, Raymond Pohler, Jon Gore, Stuart Eaton, Adrian Bowles, Tim Jarman

Issue Date: February 2005

DOE Award Number: DE-FC26-01NT41154

Submitted by:

Contractor

Tuboscope Pipeline Services

2835 Holmes Road

Houston, TX 77051

Subcontractor

QinetiQ Itd.

Cody Technology Park

Ively Road, Farnborough

Hants GU14 OLX UK

DOE Project Officer:

Daniel Driscoll

US Department of Energy National Energy Technology Laboratory (NETL) 


\section{Disclaimer:}

"This report was prepared as an account of work sponsored by an agency of the United States Government. Neither the United States Government nor any agency thereof, nor any of their employees, makes any warranty, express or implied, or assumes any legal liability or responsibility for the accuracy, completeness, or usefulness of any information, apparatus, product, or process disclosed, or represents that its use would not infringe privately owned rights. Reference herein to any specific commercial product, process, or service by trade name, trademark, manufacturer, or otherwise does not necessarily constitute or imply its endorsement, recommendation, or favoring by the United States Government or any agency thereof. The views and opinions of authors expressed herein do not necessarily state or reflect those of the United States Government or any agency thereof. " 


\section{Abstract:}

This report describes prototypes, measurements, and results for a project to develop a prototype pipeline in-line inspection (ILI) tool that uses electromagnetic acoustic transducers (EMATs) to detect and grade stress corrosion cracking (SCC). The introduction briefly provides motivation and describes SCC, gives some background on EMATs and guided ultrasonic waves, and reviews promising results of a previous project using EMATs for SCC.

The experimental section then describes lab measurement techniques and equipment, the lab mouse and prototypes for a mule, and scan measurements made on SCC. The mouse was a moveable and compact EMAT setup. The prototypes were even more compact circuits intended to be pulled or used in an ILI tool. The purpose of the measurements was to determine the best modes, transduction, and processing to use, to characterize the transducers, and to prove EMATs and mule components could produce useful results.

Next, the results section summarizes the measurements and describes the mouse scans, processing, prototype circuit operating parameters, and performance for SHO scans. Results are given in terms of specifications - like SNR, power, insertion loss - and parametric curves such as signal amplitude versus magnetic bias or standoff, reflection or transmission coefficients versus crack depth. Initially, lab results indicated magnetostrictive transducers using both SH0 and SV1 modes would be worthwhile to pursue in a practical ILI system. However, work with mule components showed that SV1 would be too dispersive, so SV1 was abandoned.

The results showed that reflection measurements, when normalized by the direct arrival are sensitive to and correlated with SCC. This was not true for transmission measurements. Processing yields a high data reduction, almost 60 to 1, and permits $A$ and $C$ scan display techniques and software already in use for pipeline inspection. An analysis of actual SHO scan results for SCC of known dimensions showed that length and depth could be determined for deep enough cracks. Defect shadow and short length effects were apparent but may be taken into account. The SHO scan was done with the mule prototype circuits and permanent magnet EMATs. These gave good enough results that this hardware and the processing techniques are very encouraging for use in a practical ILI tool. 


\section{TABLE OF CONTENTS}

Abstract 2

Table of Contents 3

$\begin{array}{ll}\text { Abbreviations } & 4\end{array}$

Introduction $\quad 5$

Executive Summary $\quad 11$

Experimental and Developmental 13

Results and Discussion 31

Conclusion and Summary $\quad 55$

References $\quad 59$ 


\section{Abbreviations}

A

A Scan

C Scan

CW

CCW

DOE

EDM

EMAT

$f-t$

$\mathrm{Hz}$

ILI

$\mathrm{KHz}$

m

$\mathrm{mm}$

$\mathrm{nV}$

ML

MOSFET

ppm

rms

SCC

$\mathrm{SHO}$

SNR

sqrt

SV1

V
Amperes

Analog scan

Contour scan

Clockwise

Counter clockwise

United States Department of Energy

Electrostatic discharge machining

Electro magnetic acoustic transducer

Frequency-time

Hertz

In line inspection

kilo-Hertz

meter

mili-meters

nano-Volt

Meander line

Metal oxide on silicon field effect transistor

Periodic permanent magnets

root mean square

Stress corrosion cracking

Shear horizontal mode 0

Signal to noise ratio

Square root

Shear vertical mode 1

Volt 


\section{Introduction}

In past years, concern has grown in the pipeline industry about Stress Corrosion Cracking [1] (SCC), a complex phenomenon where colonies of tiny cracks form on steel pipe surfaces. The fact that it is a significant issue for pipelines, was reinforced by a major disruption of gasoline in Arizona in 2003, a conference of operators in December 2003, and a survey in 2004. SCC can grow over a period of years and eventually lead to pipeline rupture. Consequently, it is important to detect, monitor and grade SCC to prevent pipeline failures. SCC presents various challenges because the cracks are very narrow and axially oriented and occur in gas lines, so that conventional magnetic and liquid coupled ultrasonics do not work.

An EMAT Electromagnetic Acoustic Transducer [2, 3] based system is a possible means to monitor SCC. In this technique, coils and permanent magnets are located in close proximity to, but not in contact with the surface of the material. The coils are excited with radio frequency (RF) current and create high frequency eddy currents or magnetic fields in the material. These interact with a static magnetic bias and produce mechanical strains in the material and hence give rise to ultrasonic waves. The waves can be used for detecting and measuring defects, cracks and flaws by transmission and reflection analyses [4, 5].

By way of review there are two basic types of EMATs: magnetostrictive $[6,7]$ and Lorentz $[2,3$, 8]. These are diagrammed in Figures 1 and 2. A magnetostrictive EMAT uses the fact that pipe materials undergo magnetostriction, which means that they stretch under a magnetic field and shrink to normal size when that field is removed. The coil creates a high frequency magnetic field in the pipe wall, which in turn creates waves by the stretching and shrinking the pipe material. In a magnetostrictive EMAT the magnetic bias field linearizes, strengthens, and directs the strain effects. This field is in the plane of the pipe wall, and the bias magnets can be separated from the RF coil. In a Lorentz EMAT, the coil creates eddy currents in the wall, which then create forces in the wall via Lorentz interaction with the bias field. In the Lorentz EMAT, the bias field is perpendicular to the pipe wall, and the magnet must be over the coil.

For cracks the ultrasonic energy of interest that EMATs excite consists of guided modes or waves, referred to as Lamb waves $[9,10]$. These guided modes can have particle motions (or strains) that are horizontal $(\mathrm{SH})$ or vertical (SV) to the direction of travel. The modes are mainly confined to the steel of the pipe and can travel for long distances. For axial cracks, circumferentially travelling modes are of the most interest because they will broadside the SCCs and interact most strongly with them.

Most guided modes are dispersive in that speeds vary with frequency, which causes a pulse of energy to lengthen with time and distance. The dispersion curves in pipe (also plates) depend on the wall thickness, such that the speed variations for a given mode are a function of frequency multiplied by thickness [5]. Because of this modes are specified by frequency thickness product (i.e. $\mathrm{MHz}-\mathrm{mm}$ ) and not just by frequency, so that modal properties are relevant to any wall thickness.

Although EMATs are a possible approach to SCC in gas lines, at the start of this project there remained significant questions concerning which type of EMAT and which modes to use and then how to optimise the transducer parameters, 


\section{Magnetostrictive EMAT}

(Planar magnetic bias + magnetically induced strains)

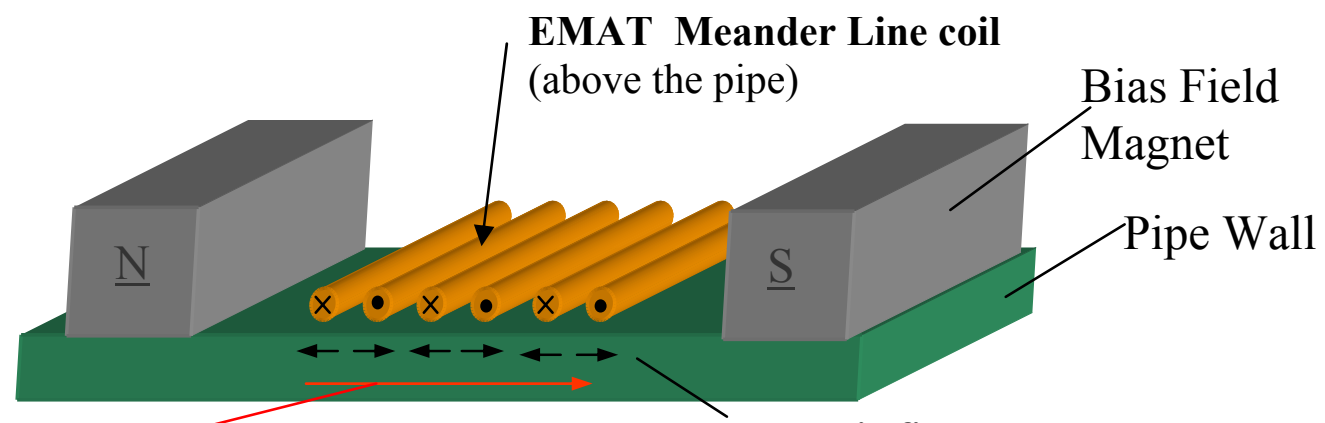

Bias field, $\mathrm{H}_{\mathrm{b}}$

Dynamic field, $\mathrm{H}_{\mathrm{d}}$ Induced By the Coil

(in the pipe wall plane, Magnetostrictive Strains axial or circumferential)

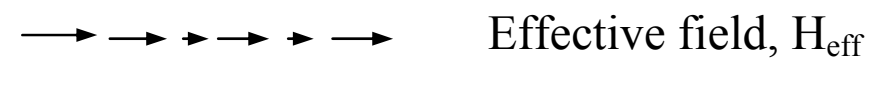

Figure 1 Structure of a Magnetostrictive EMAT Using a Meander Line Coil

\section{Lorentz EMAT}

Perpendicular bias + strains from Lorentz interactions with eddy currents

EMAT Meander Line coil (above the pipe)

Eddy current paths (induced in the pipe wall)
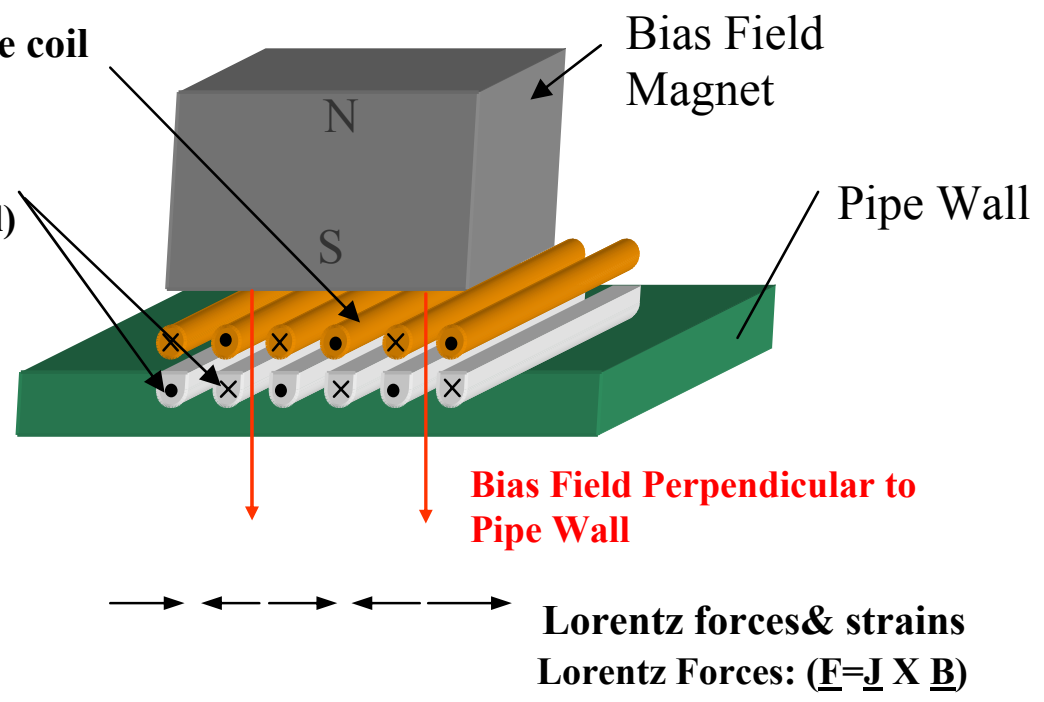

Figure 2 Structure of A Lorentz EMAT Using a Meander Line Coil 
such as magnetic biasing, coil length, width, and impedance. There was a lack of understanding about the nature of the acoustic wave interactions with the multiplicity of crack and defect types that could be encountered in gas transmission pipelines. Finally, there were many difficult practical problems concerning ruggedness, power consumption, false alarm and error performance. This project sought answers to these questions and to address the problems.

Previously, a project $[11,12]$ was completed for the Gas Technology Institute (GTI) which at first concerned a multimode EMAT device and later detailed investigations of single mode EMATs, in which modelling for guided modes was done for dispersions, for modal shapes, for the reflection and transmission from simple cracks and for the attenuations from the exterior coating and back fill. From this, six modes (at specific frequencies) appeared to be good candidates for further lab study. These had the following desirable properties: low dispersion, high group velocity, high sensitivity to cracks, and low (modelled) attenuation due to the coating. They consisted of two SV modes and three SH modes. They are listed in Table 1.

\section{Table 1 Promising Modes to Pursue For an ILI SCC Tool}

\begin{tabular}{|c|c|c|c|c|c|c|c|c|c|}
\hline \multicolumn{10}{|c|}{ Promising $s V$} \\
\hline Mode & $\mathrm{f}-\mathrm{d}(\mathrm{MHz}-\mathrm{mm})$ & $\mathrm{f}(\mathrm{MHz})$ & $\mathrm{wl}(\mathrm{mm})$ & $V p(m / s)$ & $\mathrm{Vg}(\mathrm{m} / \mathrm{s})$ & Attn $1(\mathrm{~dB} / \mathrm{m})$ & \multicolumn{3}{|c|}{$\begin{array}{c}\text { Attn2 }(\mathrm{dB} / \mathrm{m}) \quad \operatorname{Disp}(\mathrm{Y} / \mathrm{N} \\
\text { Wavelen }(\mathrm{mm})\end{array}$} \\
\hline sV1 & 3.91 & 0.47 & 12.81 & 5956.00 & 4949.00 & 9 & 18 & 12.7 & $\mathbf{N}$ \\
\hline sv2 & 7.78 & 0.93 & 6.44 & 5959.00 & 4943.00 & 35 & 65 & 6.4 & $\mathbf{N}$ \\
\hline \multicolumn{10}{|c|}{ Promising $\mathrm{SH}$} \\
\hline Mode & $\mathrm{f}-\mathrm{d}(\mathrm{MHz}-\mathrm{mm})$ & $\mathrm{f}(\mathrm{MHz})$ & $\mathrm{wl}(\mathrm{mm})$ & $V p(m / s)$ & $\operatorname{Vg}(\mathrm{m} / \mathrm{s})$ & Attn $1(\mathrm{~dB} / \mathrm{m})$ & \multicolumn{3}{|c|}{$\begin{array}{c}\operatorname{Attn} 2(\mathrm{~dB} / \mathrm{m}) \quad \operatorname{Disp}(\mathrm{Y} / \mathrm{N}) \\
\text { W avelen }(\mathrm{m} \mathrm{m})\end{array}$} \\
\hline SHO & 0.67 & 0.08 & 40.75 & 3260.00 & 3260.00 & 2 & 14 & 40.7 & $\mathbf{N}$ \\
\hline SHO & 1.51 & 0.18 & 18.11 & 3260.00 & 3260.00 & 8 & 18 & 20 & $\mathbf{N}$ \\
\hline SHO & 2.14 & 0.26 & 12.78 & 3260.00 & 3260.00 & 3 & 8 & 12.5 & $\mathbf{N}$ \\
\hline SH1 & 2.24 & 0.27 & 17.50 & 4673.00 & 2273.00 & 10 & 23 & 20.9 & $\mathbf{Y}$ \\
\hline
\end{tabular}

$f-d=$ frequency-thickness product; $f=$ frequency of excitation in $8.4 \mathrm{~mm}$ thick plate; $w \mathrm{l}=$ wavelength; $V p=$ phase velocity; Vg = group velocity; Attn1 = viscoelastic attenuation in plate with a $2 \mathrm{~mm}$ coating of coal tar epoxy; Atth 2 = leakage and attenuation in a coated pipe buried in clay (modelled worst-case scenario); Disp (Y/N) = indicated whether or not the mode exhibits dispersion at the excitation frequency.

Their 2D FEM modelled dispersion curves and modelled crack interactions are shown in Figures 3 (for SV) and 4 (for SH). 

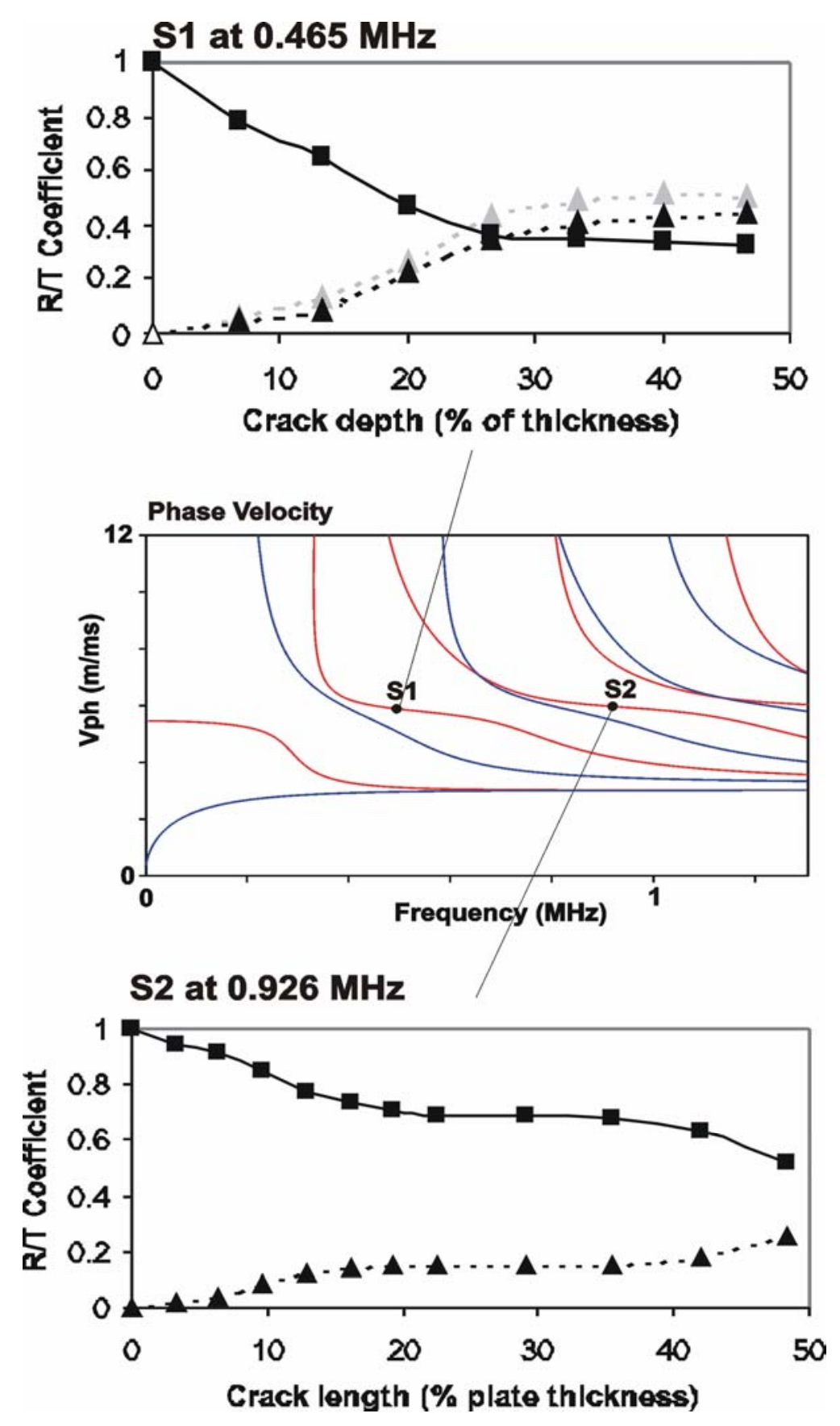

Figure 3 Modelled dispersion curves and crack interactions for SV1 and SV2 modes. 
SHO at $0.18 \mathrm{MHz}$

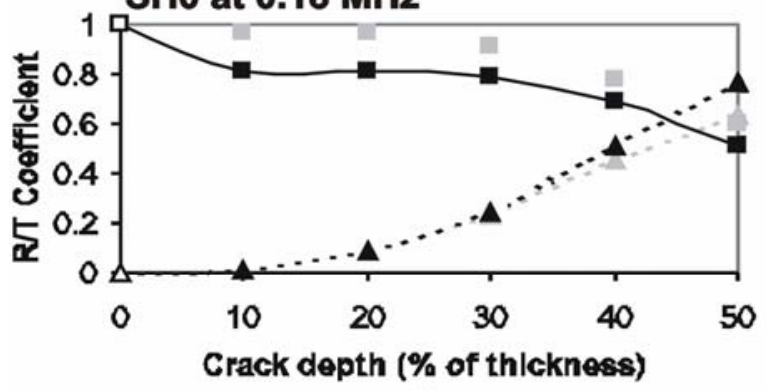

SH1 at $0.267 \mathrm{MHz}$

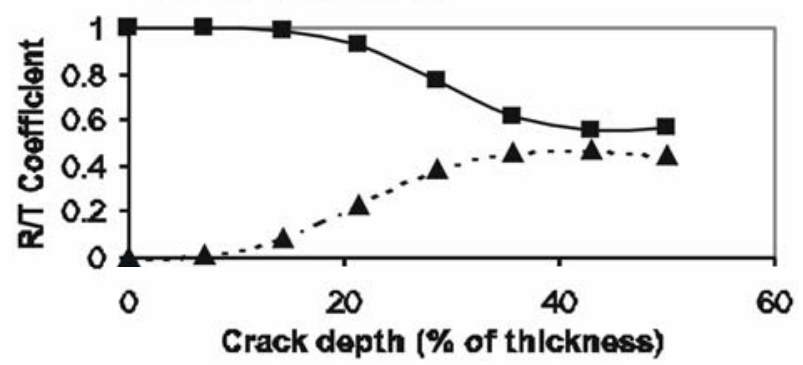

SH mode phase velocity spectrum for $8.4 \mathrm{~mm}$ thick steel plate

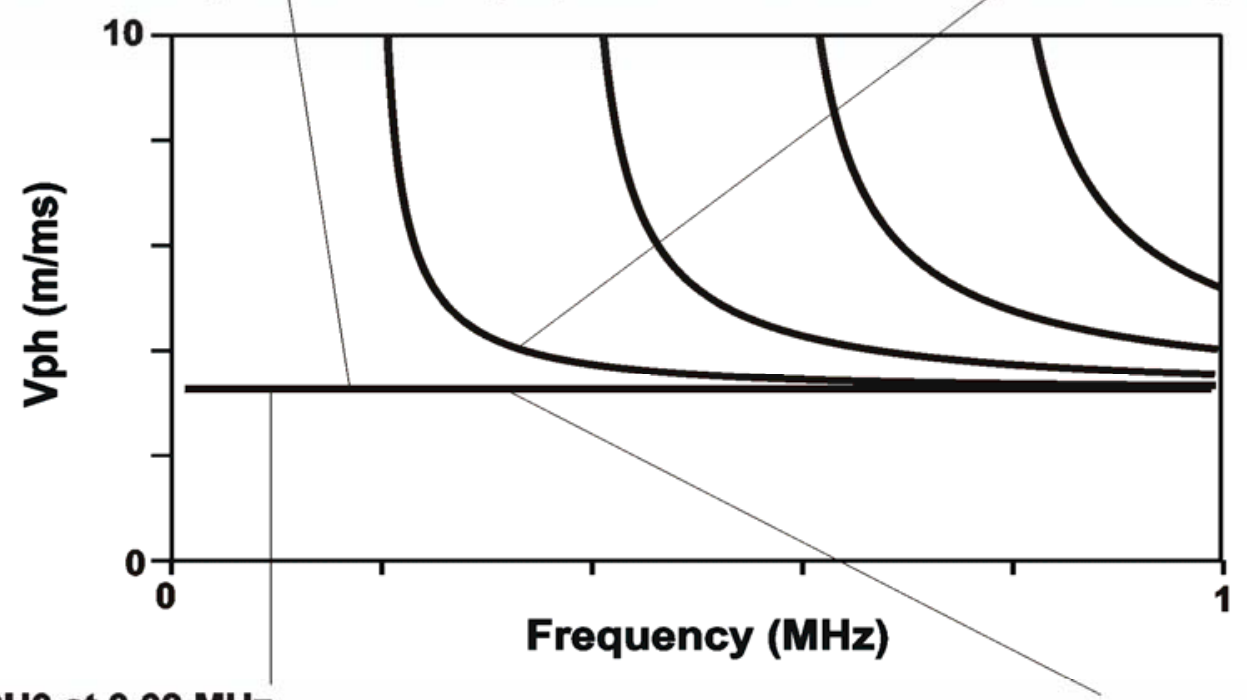

SHO at $0.08 \mathrm{MHz}$

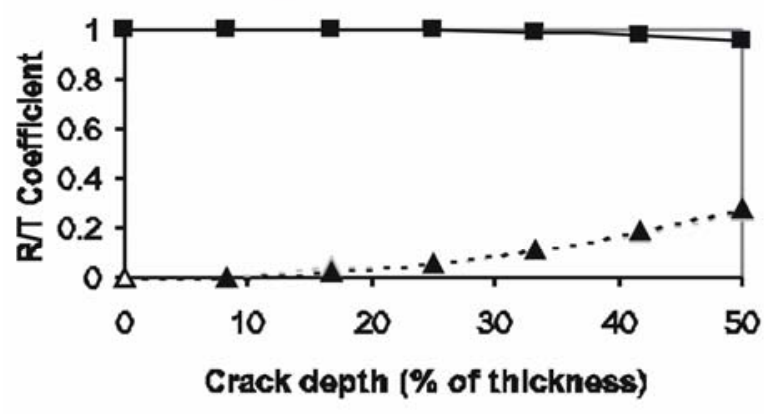

$\mathrm{SHO}$ at $0.255 \mathrm{MHz}$

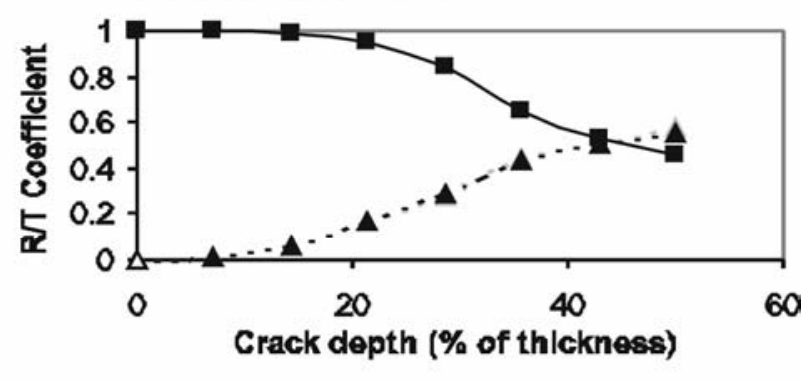

- - - - - Reflection Coef Reflection Coef (time domain)

Figure 4 Modelled dispersion curves and crack interactions for SHO and SH1 modes. 
Also as part of the GTI work, a lab system was assembled and an EMAT transmitter for only one mode was built and tested with a piezo receiver. With that system no EMAT receiver was successfully operated and only the SV2 mode had reasonable signal level (with a piezo receiver). However, crack interaction with the single mode was verified, albeit at a low signal to noise ratio. It was clear that significant improvements had to be made in the lab system for the DOE project. This is where this DOE project started, and this report picks up.

In this work for DOE, in order to develop a suitable commercial system, the method was first to do lab experiments of increasing scale and complexity to find the best modes and frequencies and transducing techniques (magnetostrictive or Lorentz), and to improve basic transducer responses, and then to change to a more complicated device (lab mouse) that could be manually pulled through a pipe. The next step was to change again to a device (a mule) that was more rugged, closer to commercial, and that could be pulled rapidly in a longer pipe, and simply modified into a full size commercial prototype.

This report encompasses work from the beginning of October 2001 until the end of September 2004. It covers lab measurements of increasing size and complexity to identify the best acoustic modes and transducing methods, improvements to transducers and equipment, a review of the lab mouse, the lab mouse results on SCC pipe, descriptions of mule and ILI components, scan results with mule components, processing for a tool or mule that are indicated by the data acquired with mule components.

In addition to this introduction, this report consists of three major sections. An experimental and developmental section describes the lab measurements, the lab mouse, its application, and work done on refinements and mule components to make an ILI tool practical. The results and discussion section describes the mouse results on SCC pipe, scan results with prototype mule components on man-made cracks of known dimensions, and processing that can simplify a real tool. The report ends with a section that summarizes the report, important conclusions, and points out future work. 


\section{Executive Summary}

This report describes experiments, and results for a project to develop a pipeline in-line inspection tool that uses electromagnetic acoustic transducers (EMATs) to detect and grade stress corrosion cracking (SCC). There is a brief introduction that gives background material about EMATs and relevant previous work [12] for the Gas Technology Institute. This work left six choices for the acoustic modes and a choice between magnetostrictive or Lorentz type transducers for this project.

The experimental section at first describes the lab systems, improvements to these systems, and setups and techniques to narrow the choice of modes and transduction. Improvements, which involved transducer matching networks, better magnetic biasing, and lower noise electronics, led to improved signal to noise (SNR) ratios. The setups permitted transducer characterizations and interaction measurements in plates with man-made cracks, pipeline sections with SCC, and a full pipe with SCC. The latter were done at first with a lab mouse and then mule components. The mouse could be pulled slowly, and the mule components were compact and efficient enough to be used in an ILI tool.

The experimental and developments section describes the lab mouse, its use on the SCC pipe, and mule components. For the lab mouse two separate carriers (one for SH0 and one for SV1) were built and deployed on real pipe with some SCC defects. Each mouse had a pitch-catch arrangement with electromagnets. The orientation of the bias magnets and the drive frequency determined if SH0 or SV1 was logged. These units were pulled through the pipe and stationary waveform acquisitions were made for reflection and transmission through SCC. All major support equipment for the mouse consisted of large units, such as signal generators, high wattage supplies, and a digitizing scope. Mule components consisted of permanent magnet EMATs, compact preamps and a filter, compact power supply modules, and a class D (pulse) power amp. These considerably shrunk mouse support equipment and increased performance for a mule or prototype ILI tool. For example, a simple gate array replaced a signal generator box. The pulse power amp increased peak power output current to more than 60 amps from 25 of the mouse.

The results section justifies the mode and transducer choices. At first these were for magnetostrictive EMATs and the use of EMAT launched modes: SH0 (at 2.1 MHz-mm) and SV1 (at $3.9 \mathrm{MHz}-\mathrm{mm}$ ). In general, the improvements and measurement results impacted this choice. The main justifications were good SNRs and useful crack sensitivity. Later SV1 was dropped due to its dispersion that would distort signals if wall thickness varied a moderate amount. SHO was shown to have minimal or no dispersion.

The results section then gives details of measurements on these modes. The measurements consisted of signal to noise ratio, insertion loss, magnetic biasing sensitivities, crack reflection and transmission coefficients, beam width, standoff and tilt sensitivities. In most cases the section presents useful parametric curves - for example, reflection coefficient versus crack depth. The section shows scan results for a pipe section having SCC and mouse waveforms from a full pipe in areas with and without a defect. This section also reviews further coil optimisation and implementation issues, such as power, acquisition parameters, and magnetic configuration.

The results section describes mouse results and processing that could be used in a prototype ILI tool. The results show promise for reflection measurements that are normalized and contraindicate transmission measurements. The normalized reflection measurements correlate well 
with the SCC and repeat. The processing consists of jittering the firing rate, averaging, digital envelope extraction, filtering, and down sampling. These allow data and memory reduction in the tool. They are simple enough to implement in a tool by means of a gate array or DSP. They also allow stored data to be channelized, so it can be displayed and interpreted in a similar way to MFL data. Examples are shown.

Finally, conclusions about the development, mouse and mule results, and processing are summarized. The significant conclusions are:

- Magnetostrictive EMATs were chosen because they produced similar and useable SNRs to Lorentz, yet they would be more robust and easily implemented in an ILI tool.

- Magnetostrictive EMATs were successfully built for a mouse and a mule and achieved good scan results on real pipe.

- $\mathrm{SHO}$ and SV1 reflection measurements are sensitive to SCC.

- Transmission measurements are not sensitive to SCC. This is due to large variations in amplitude.

- Thickness and dispersion affect SV1 measurements, and make it difficult to excite and use over a broad range of wall thicknesses. This does not happen for $\mathrm{SHO}$.

- For commercial application and the mule because of the above, SV1 was abandoned and $\mathrm{SHO}$ was pursued in depth.

- Beam spreading and lift-off effects for the wavelength to be used ( 12 mm) are moderate for moderate range (a few feet) and a few millimetres of lift-off.

- $\mathrm{SHO}$ attenuation is small for a few feet of range and so tolerable, even for a round trip around a 30" pipe.

- SHO reflection measurements can be done on extracted envelopes that must be normalized by the direct arrival amplitude. These so called, normalized reflections, can be treated like reflection coefficients.

- Crack axial lengths and depths can be estimated for moderate to deep cracks from SHO normalized reflection measurements. Crack width cannot be estimated very well.

- Compact circuits and permanent magnet EMATs for the receiver and transmitter are suitable for a mule and prototype tool work and provide good measurements. This is evidenced by clear and useful $A$ and $C$ scan results on a pipe with man-made SCC.

- Processing was developed that successfully channelizes the data for use with Tuboscope LinaView software and analog and contour scan (A and C scan) displays. This processing also results in large data reductions and is amenable to implementation with available chips within an ILI tool.

- In sum, the results were by a large majority positive and thus very encouraging to proceed with magnetostrictive EMATs and $\mathrm{SHO}$ toward a commercial prototype. 


\section{Experimental and Developmental}

\section{Flat Plate Lab Set up and Improvements}

Work was initially done to improve the lab system from the GTI project. The final, improved, lab system, is shown in Figure 5 and diagrammed in Figure 6. Improvements over the GTI system consisted of the following: EMAT receivers, efficient impedance matching networks for transmitters and receivers, new magnetic bias magnets and a bias supply that yielded increased magnetic biasing fields (more amp-turns) and more uniformity of magnetic bias, a new preamplifier that reduced noise and firing breakthrough, new EMAT coils with reduced losses, and complete grounding and shielding that further reduced noise. This was then used for the following measurements or investigations on a flat plate of pipeline steel: transmitter output and sensitivity variation with magnetic biasing, receiver sensitivity, signal to noise ratio, pitch/catch insertion loss, crack interactions (reflection and transmission coefficients).

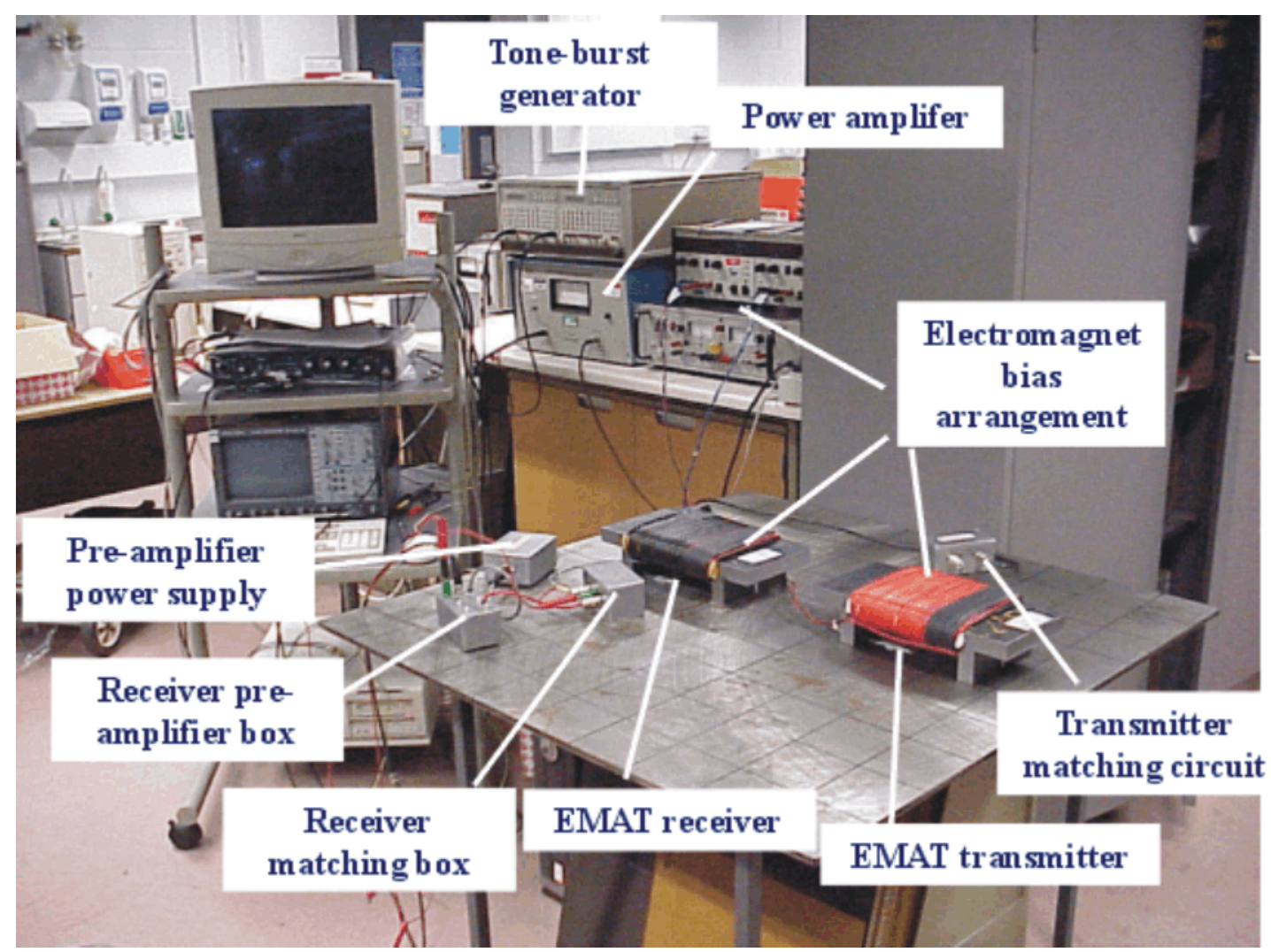

Figure 5 Laboratory set-up of the pitch-catch experiments using SV1 EMAT heads biased for magnetostrictive coupling. 


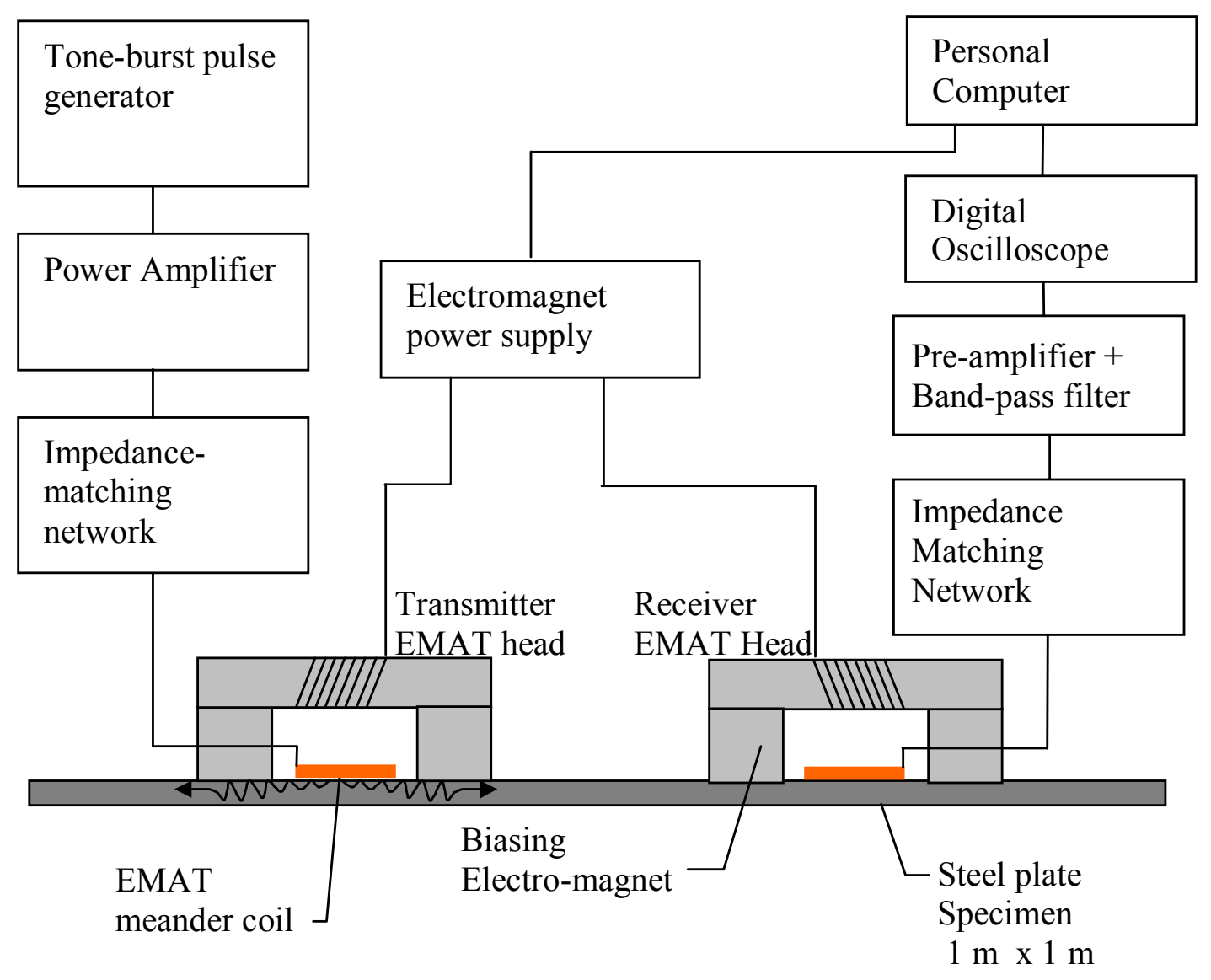

Figure 6 Schematic of the lab set-up used in most experiments (in pitch-catch configuration)

This system, had separate EMAT heads and electro-magnet biasing for both the transmit and receive heads and operated in pitch-catch mode. This approach not only simplified the electronic circuit design but was also necessary if the optimum biasing was different for transmitters and receivers.

For all flat plate measurements the plate was one meter square and $8.4 \mathrm{~mm}$ thick of pipeline steel. It was supported at the edges by wooden legs.

The new biasing electromagnets carried about 800 turns, were capable of 10,000 Amp-turns and rested on the plate The DC supply, which was computer controlled, allowed the plate and the poles to be degaussed before any measurements were taken. Degaussing was done for all flat plate measurements. It became apparent during early tests that magnetostrictive EMATs required an axial magnetic bias for $\mathrm{SHO}$ and a circumferential for SV waves. This is diagrammed in Figure 7.

The EMAT (Figure 8) coils were initially flat, minimal resistance, single layer meander line (ML) patterns of 5 periods ( $80 \mathrm{~mm}$ wide by $60 \mathrm{~mm}$ ) that fit between the poles of the bias magnets. 


\section{Static Magnetic Biasing For Magnetostrictive EMATs}

SV Modes

Circumferential Magnetic Bias

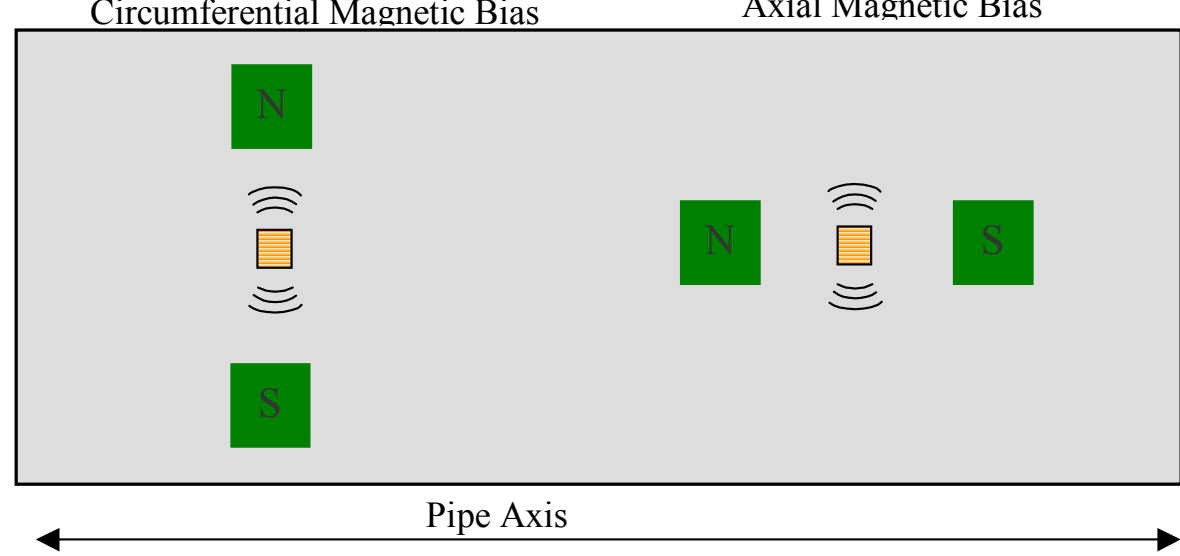

SH Modes

Axial Magnetic Bias

Pipe

Cicumference

Figure $7 \quad$ Magnetic bias field geometry relative to wave propagation For magnetostrictive EMATS

Flexible heads, which are necessary for curved specimens such as pipe sections, were later produced by etching polyimide-backed copper of $70 \mu \mathrm{m}$. Some investigations were also done with periodic permanent magnet (ppm) EMAT heads and pancake coils.

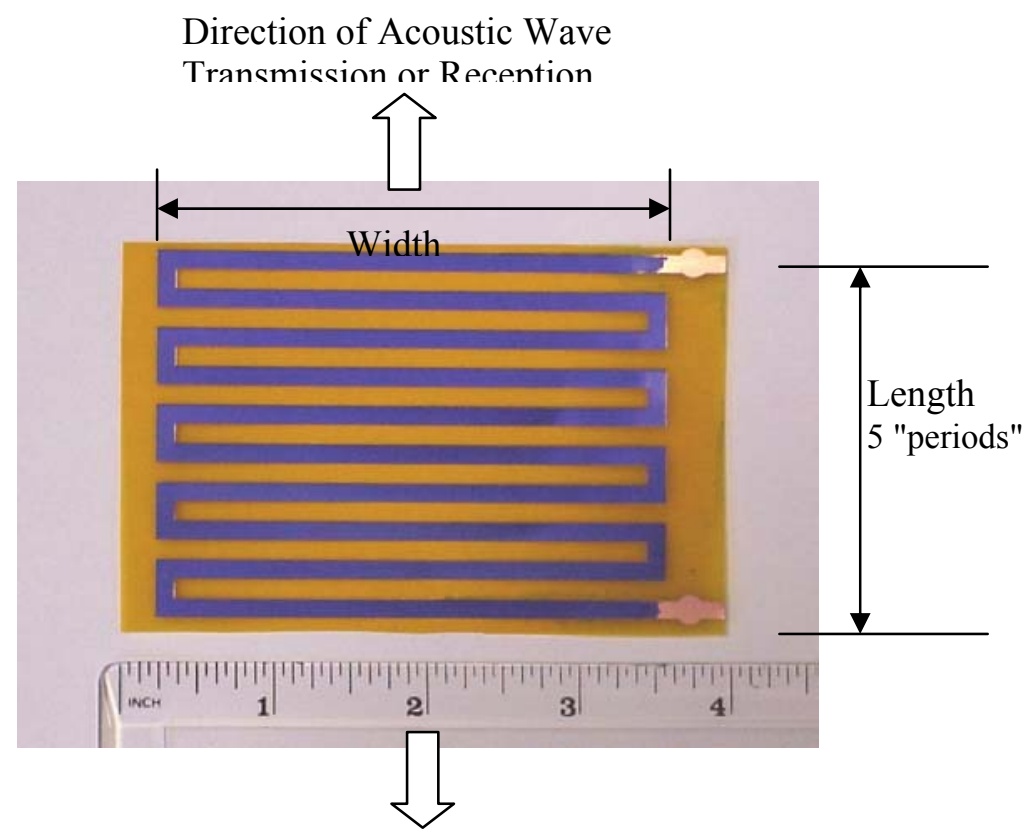

Figure 8 Example of the meander line (ML) EMAT coil used in this work.

A general purpose lab RF power amp could supply more than 35 A (after matching) of frequencies from $100 \mathrm{kHz}$ to $1 \mathrm{MHz}$. Because the power amp had a fixed 50 ohm output impedance, while the EMAT was $1 \mathrm{ohm}$ or less, a matching network was required to efficiently couple the amp to the EMAT. Without good matching, the amp would not supply sufficient 
current to the EMAT, and signal amplitude, which is proportional to transmitter current, would be degraded. For maximum power transfer the EMAT was coupled to the power amp through a shielded LC matching network. This was resonant, so a different network was made for each frequency investigated. For all measurements the EMAT was driven by a five cycle tone burst at the center frequency of the mode being investigated.

Note that there is also matching requirement for the receiver to the input preamplifier. In this case the matching network steps up the impedance and voltage of the source, so as to match an optimum impedance for the noise generated in the circuits of the preamplifier. The preamp required a match to $100 \mathrm{ohms}$, which was done by a different resonant LC network for each frequency.

The special low noise, high gain preamp was vital for improvement of signal to noise ratios. The preamp, which was developed, provided $90 \mathrm{db}$ gain followed by high and low pass (6 pole) active filters. The latest design had a switched center frequency (either $260 \mathrm{kHz}$ or $470 \mathrm{kHz}$ ) and band width. This was packaged in a shielded box with extra supply filtering.

\section{$\underline{\text { Plate and Small Section Measurements and Investigations }}$}

Crack Interaction and Modal Content

In all cases arrival times and dispersion of the captured signal was analysed to verify that it represented the desired wave mode. For initial crack interactions, reflection and transmission coefficients were measured from a long, man-made crack in a flat plate, a situation like that modelled in Figures 3 and 4, so that theory could be compared to measurements. Figure 9 shows the geometry.

All amplitude measurements were based on peak to peak measurements of digitized received signals. For example, for reflection coefficients, the direct arrival and reflection wavelets were identified in a received waveform by means of their arrival times, based on the mode group velocity and distance to crack. Then their peak to peak amplitudes were measured. The reflection coefficient was taken as the ratio of reflected peak to peak divided by the direct arrival peak to peak. For a transmission coefficient, the direct arrival peak to peak for a given crack was divided by the peak to peak for no crack. Thus, no crack would have a transmission of 1.

Note that dispersion, biasing, signal to noise, insertion loss, beam width, standoff and tilt sensitivity measurements were all made with the transmission configuration (Figure 9 A without a crack) but at a different separation between transmitter and receiver heads. 


\section{A Transmission Coefficient Measurement}

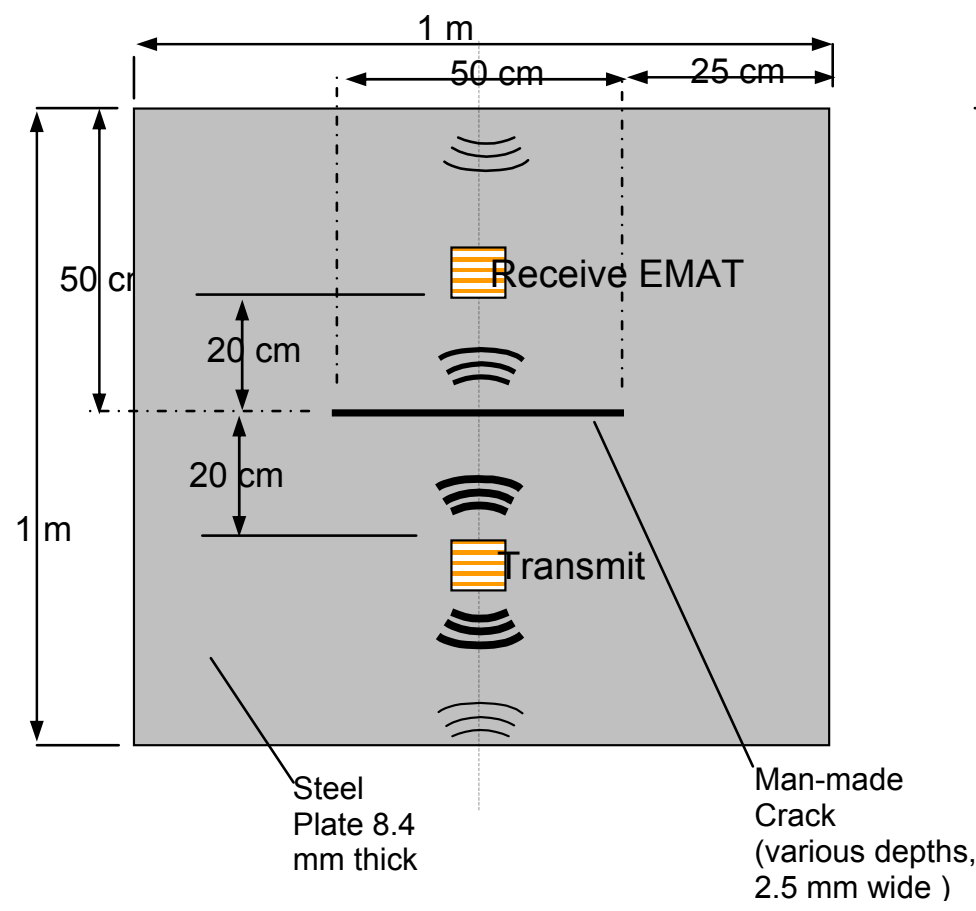

Figure 9 A Flat Plate Crack Transmission Interaction And General Pitch Catch Geometry

\section{B Reflection Coefficient Measurement}

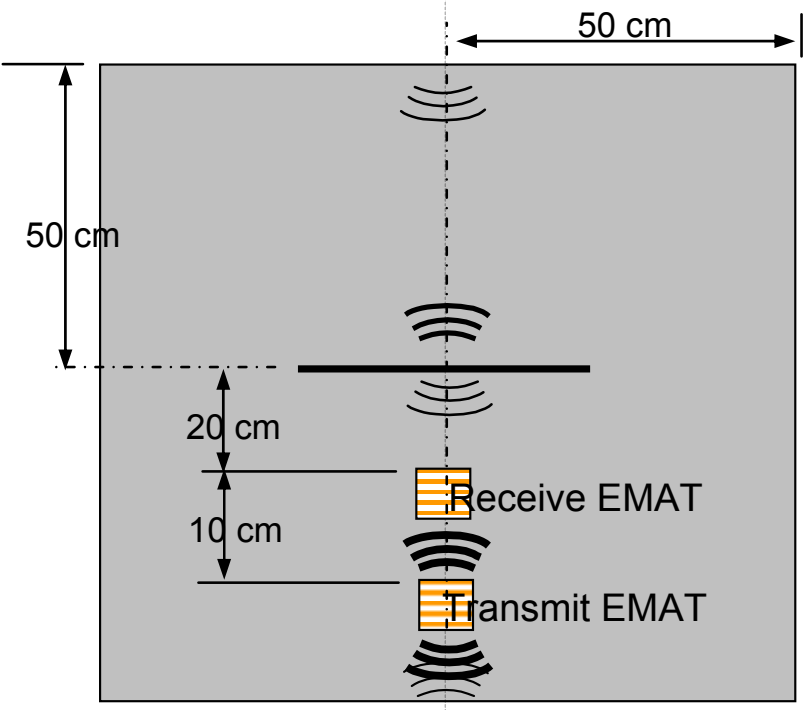

Figure 9 B Reflection Crack Interaction Geometry

Beam width at operation frequency

Two experiments were undertaken to characterise the beam width of the transducer in Figure 8. Both were done for SV1 but would be valid for SHO because the wavelength would be the same. In the first experiment, the EMAT receiver coil was moved relative to the EMAT transmitter coil, as shown in Figure 10, at increasing propagation distances.

EMAT

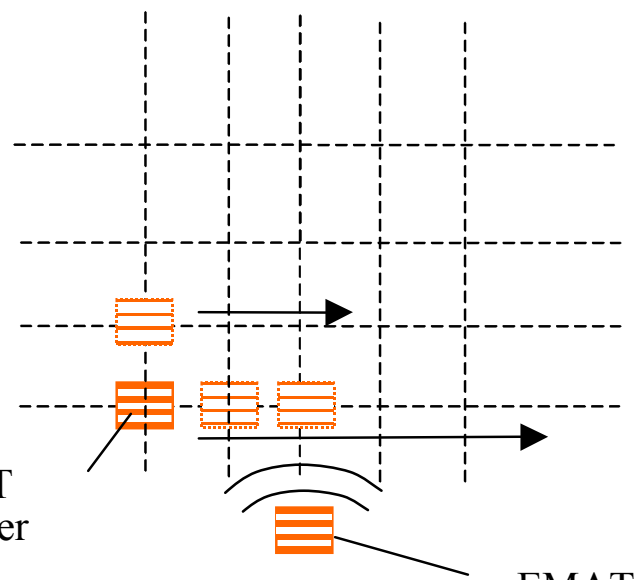

EMAT

transmit

Figure 10 Measurement of beam width of SV1 mode by receiver translation. 
In the second experiment the transmitter and receiver coils were kept in line while measurements were taken (at two different propagation distances) as the transmit coil was rotated (Figure 11).

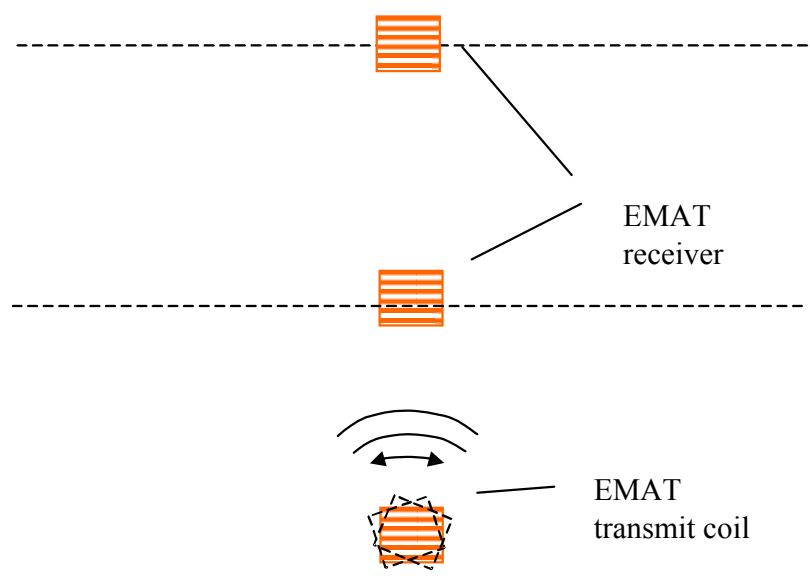

Figure 11 Measurement of angular dependency of SV1 mode by transmitter rotation

Sensitivity To Standoff and Tilt

These were done on the flat plate set up using the SV1 EMAT coil only. For these investigations the receiver and transmit coil were independently raised from the surface of the steel plate. While one transducer was lifted vertically from the plate, or tilted, the other transducer remained about $.1 \mathrm{~mm}$ from the plate surface. For tilt, one edge of the moved transducer rested $.1 \mathrm{~mm}$ from the plate while other edge was lifted. Both side to side and front to back tilting were done. We could work with SV1 only because we verified that the amplitude versus standoff depended on wavelength. Because the SHO and SV1 waves (at the frequencies used) had nearly the same wavelengths and because the coils were same, results for the SHO EMAT coil will be identical.

Determination of Best Biasing

Experiments were undertaken on a flat plate to investigate the effect of the bias field for both the transmit and receive EMAT coils on the received signal amplitude of the desired wave mode. The transmitter and receivers were set in line $20 \mathrm{~cm}$ apart on the defect-free flat steel plate. The received signal amplitude (peak to peak) was recorded as bias current for one transducer was varied (in steps), while the other was held at a fixed value. This was done for a fixed, high EMAT RF drive voltage.

\section{$\underline{\text { SCC Crack interaction Measurements }}$}

Experiments were carried out on SCC samples cut from pipe sections using bias magnets to fit the pipe. The experimental set-up is shown in Figure 12. The pipe section was cut from a 30" pipe of .312 inch wall dug for an actual pipeline due to the occurrence of SCC. The section was 1 meter axially by about .80 meters of arc. The SCC defects (actually families of small cracks) are denoted by circled areas and marked F90, F93, and F106, in the set-up geometries - Figure 13 (A) for transmission and (B) for reflection experiments. For both SV1 and SHO 
measurements, circumferential waves were launched, and the flat plate drive and acquisition system was used.

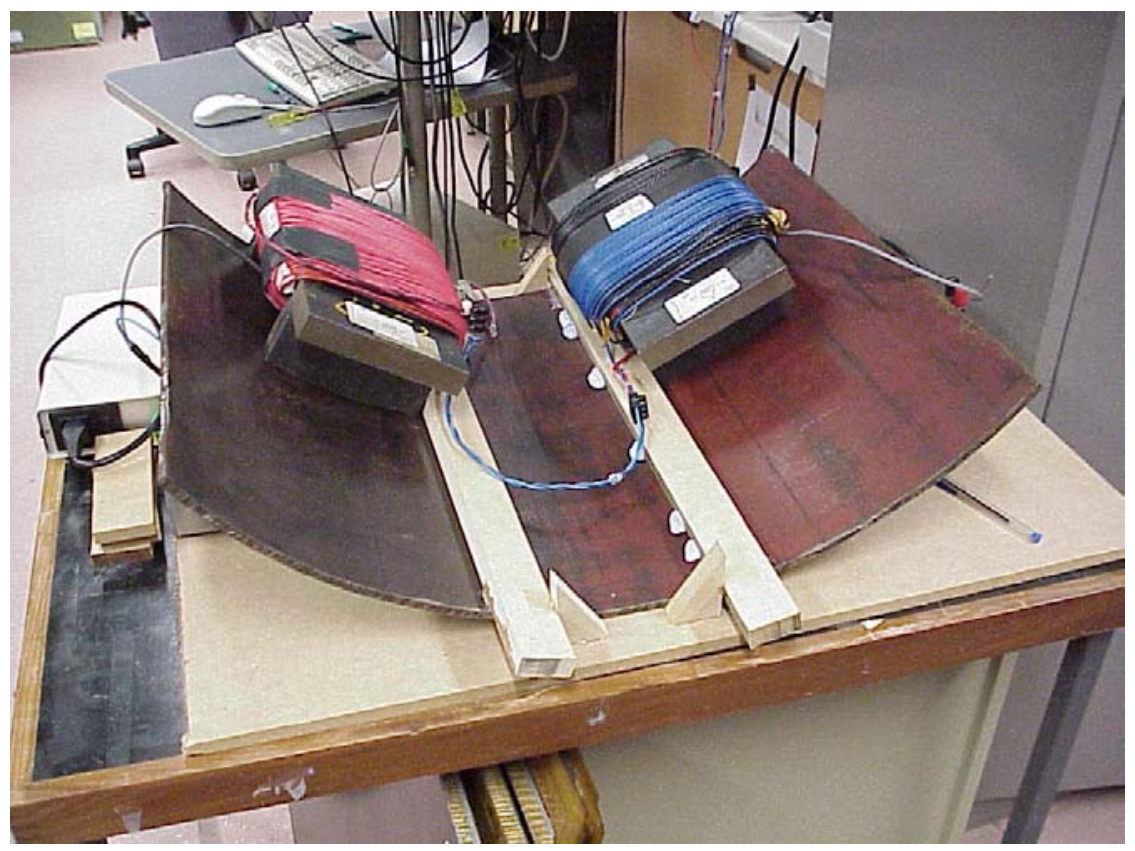

Figure 12 Experimental set-up for SCC interaction with SHO mode using pipe segments.

Waveforms were taken at 11 axial positions, with the transmitter and receiver aligned. The positions are spaced by $2.5 \mathrm{~cm}$. Time in each waveform corresponds to circumferential distance. In most cases the waveform was an average of 100 shots at the same position.

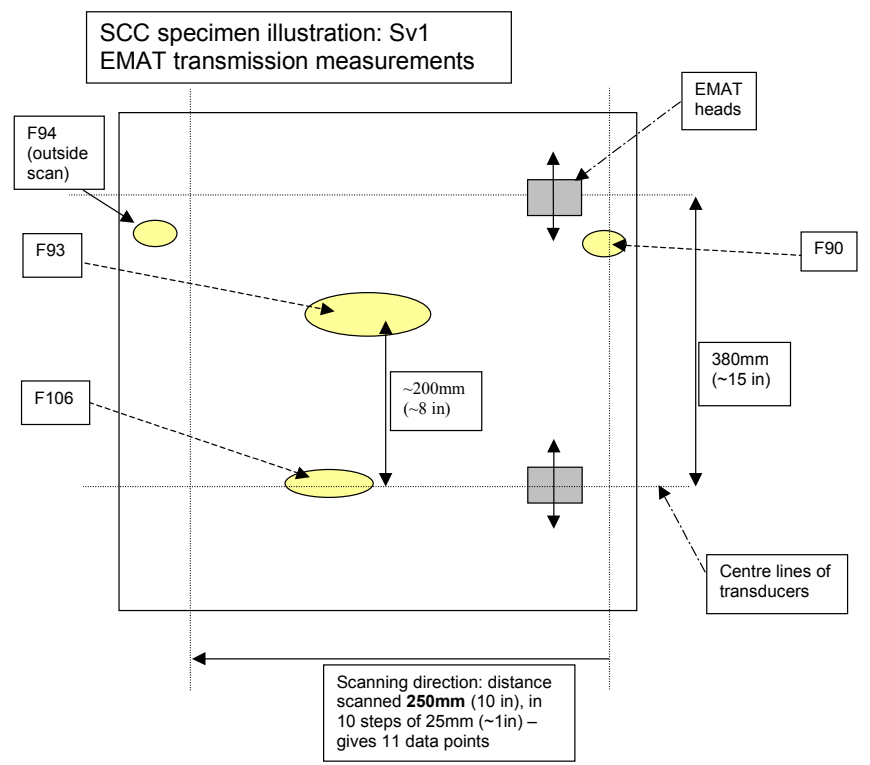

A Transmission

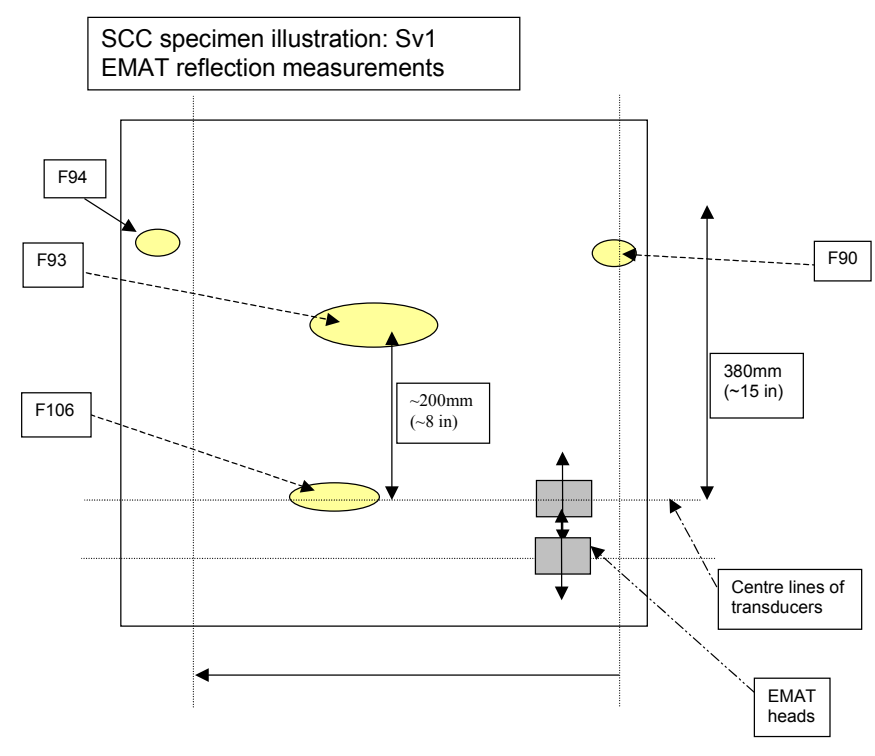

B Reflection

Figure 13 Set-ups for determining interaction of SV1 or SHO guided wave modes with SCC sites in transmission $(A)$ or reflection $(B)$ 
Table 2 Pipe Section Defect Qualification (Lengths and Depths as indicated by the pipe supplier)

\begin{tabular}{|c|c|c|}
\hline$D$ e fect ID & Approximate length $(\mathrm{m} \mathrm{m})$ & Approximate depth (\%) \\
\hline \#106 & 80 & $20-25$ \\
\hline \#93 & 70 & $15-20$ \\
\hline \# 90 & 20 & $7-10$ \\
\hline \#94 & 30 & $7-10$ \\
\hline
\end{tabular}

\section{Lab Mouse}

The lab mouse was built to allow investigation of EMAT techniques in full pipe having SCC. This device would be stepped axially along the pipe, so that the SCC defects could be scanned by the EMAT excited and received guided ultrasonic waves. Thus, EMAT responses in full pipe and SCC interactions could be evaluated. Responses for SHO and SV1 were acquired in separate passes with different EMAT pairs.

The lab mouse design used a carrier to hold biasing electromagnets and meander coils of both the transmitter and receiver EMAT heads in close proximity to the inside pipe wall and with a circumferential angular separation of 45 to 90 degrees. The coils could then be moved along the sample pipe axis on rails. Two carriers were made to hold the electromagnets for either axial magnetic field bias (SH0) or for circumferential field bias (SV1). The material of construction for the carriers and rails was chosen to be wood since it is (a) non-magnetic, (b) non-conductive, (c) highly damped, (d) versatile and (e) cost-effective. Thus, it would not support or propagate stray or confusing ultrasonic signals.

The carriers would be manually moved down the pipe axis to a sequence of stations to simulate the scan of a completed measurement system. Station measurements would be taken with the carrier, in a step, measure, and repeat fashion. The direction of wave propagation was circumferential. The mouse and associated equipment is shown in Figures 14-16. 


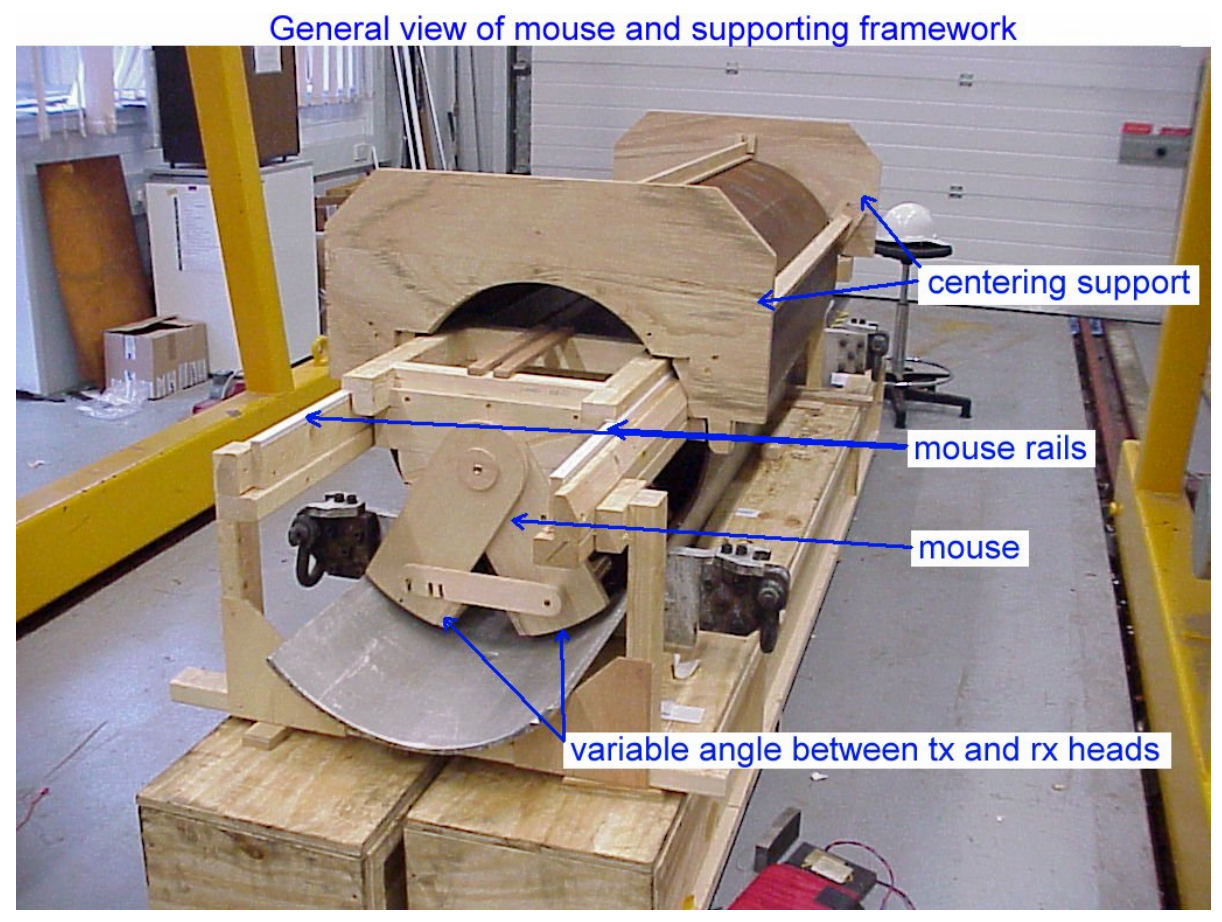

Figure 14 Overall view of the EMAT mouse set-up showing the SHO carriage being loaded into the pipe.

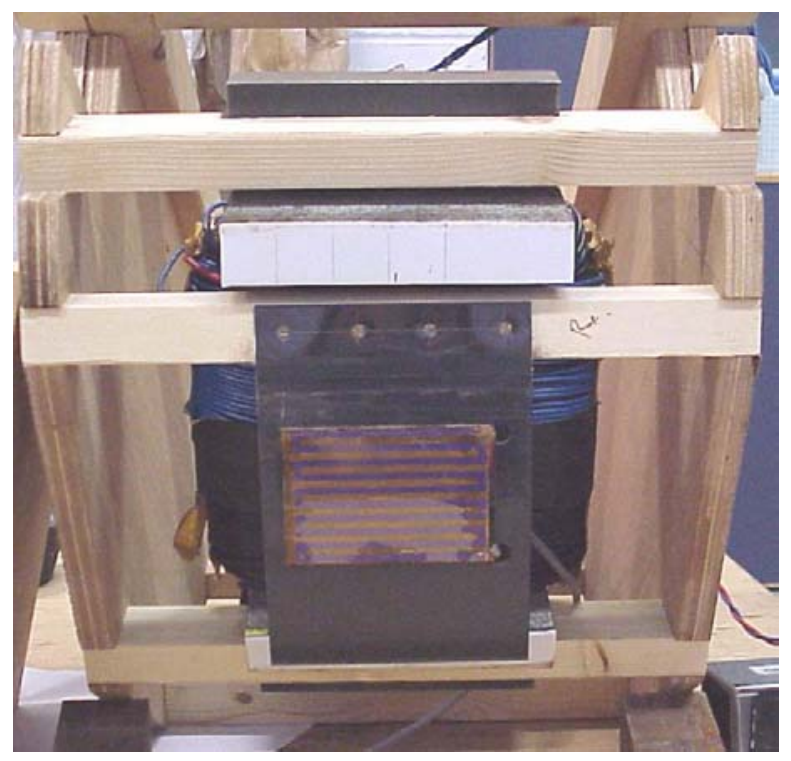

Figure 15 Side view of the SV1 carriage showing the meander line (ML) coil and circumferential biasing electromagnet in position.

For the EMAT heads two layers of polyimide backed copper meander coils $(12 \mathrm{~mm}$ period and $80 \mathrm{~mm}$ wide) in series were used. The coils were coated for protection and mounted on a polymeric substrate that had been vacuum formed to the curvature of the 24" pipe specimen being examined (pipe sample P2). The data acquisition system (Figure 16), an enhanced 
version of the flat plate system, was connected to the mouse transducers by cables long enough to allow the mouse to traverse the pipe for scans. Only the matching networks were mounted on the moving mouse. Volume for the drive/control system, excluding the monitor, EMATS and carriers, was well over 6 cubic feet, the biggest units being the DC supplies and the power amplifier.

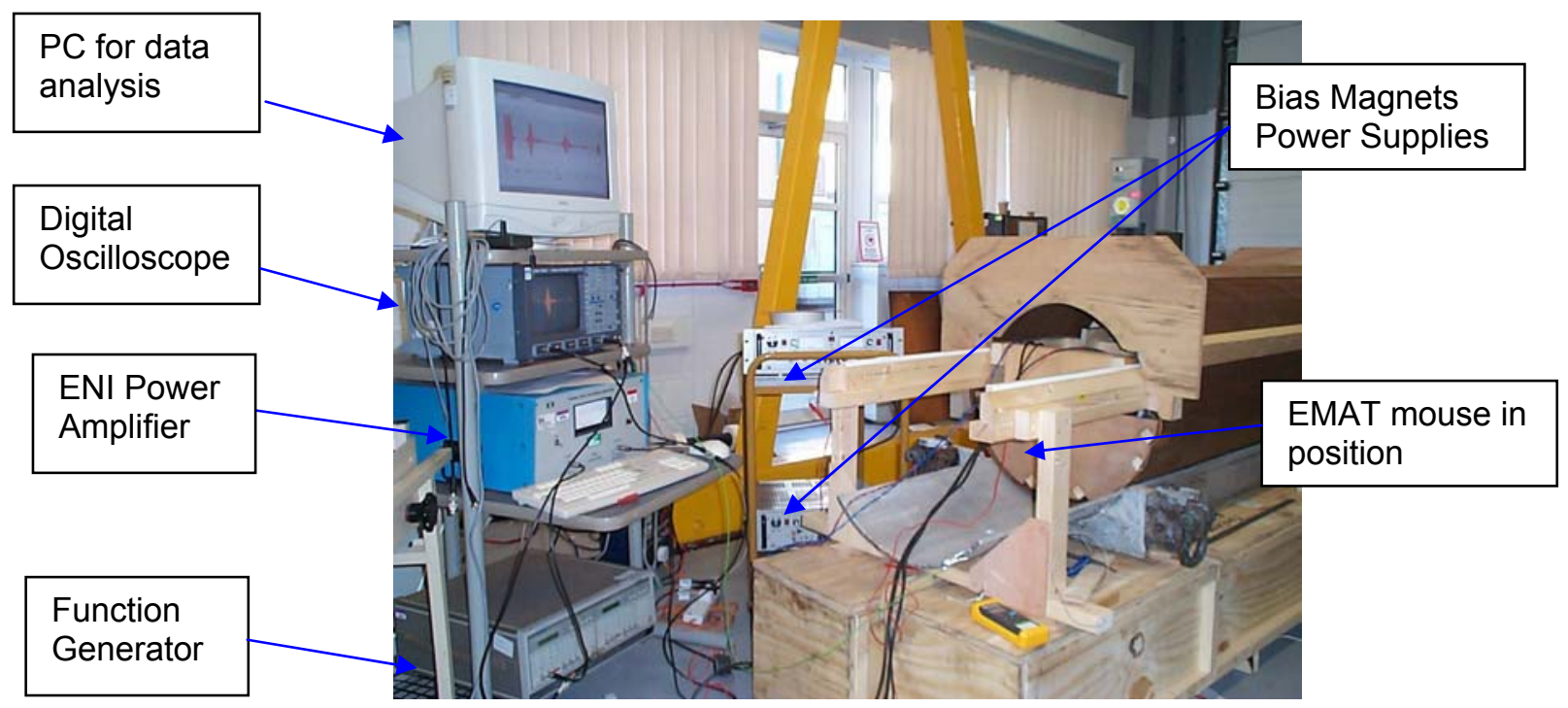

Figure 16 Overall view of the EMAT System including ancillary equipment for signal generation, data capture and data analysis.

The acquisition was completely in control of a PC, which ran Labview programs written for the mouse, in order to digitize, store, display, and analyze data. Waveforms were digitized with 5 $\mathrm{MHz}$ sampling in order to capture peak values accurately. For most cases Intervals of around 1 ms were stored in order to acquire arrivals travelling completely around the pipe.

A 24 inch diameter pipe sample with a $8.4 \mathrm{~mm}$ nominal wall thickness, designated $\mathrm{P} 2$, was chosen for the pull-through tests as (a) it was uncoated, (b) had eight defects along a line that could be clearly visually identified, but (c) otherwise appeared to be defect-free. The positions of the defects are shown in Figure 17. Conventional short wavelength shear scans [13] had mapped the defects. Defect depths were not always clear. Some defects gave strong and axially continuous reflections while others were weak and/or discontinuous. Defect P2-1 was estimated to be about $15 \%$ to $25 \%$ of wall thickness, and defect P2-4 was estimated to be shallower.

Runs were made separately for SHO and SV1. These were done with the appropriate frequency matching networks and receive filter center frequencies. For each mode both reflection and transmission scans were made.

The set-up for the reflection scans is shown schematically in Figure 18. The defect line is counter-clockwise from the receiver. In this arrangement, the receiver EMAT head $(R x)$ will first receive the direct signal (travelling counter-clockwise) from the transmitter (Tx) followed next by any reflected signals from the line of defects P2-1 to P2-8, and later the back-propagated wave from the transmitter (travelling 270 degrees around the pipe circumference clockwise). 


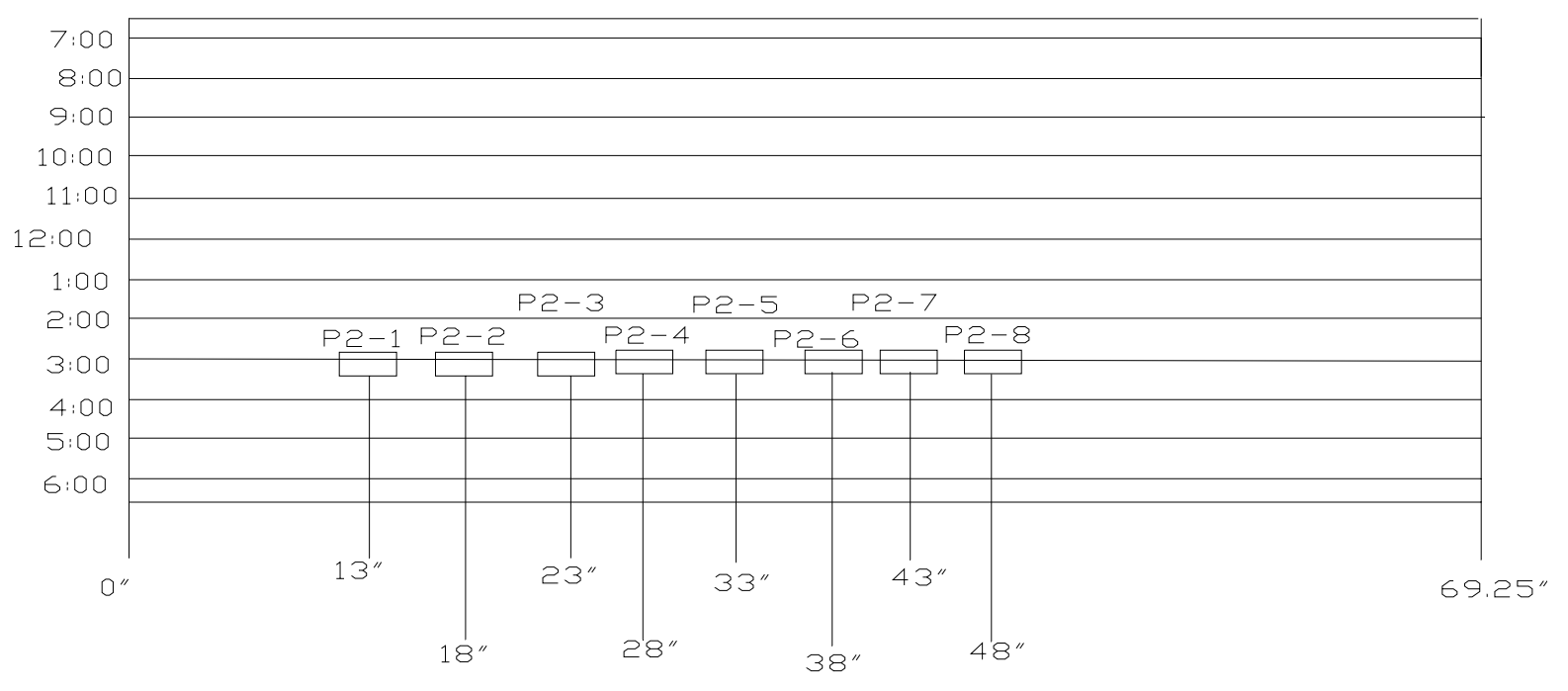

Figure 17 Positions of the defects in pipe sample P2, used in mouse measurements.

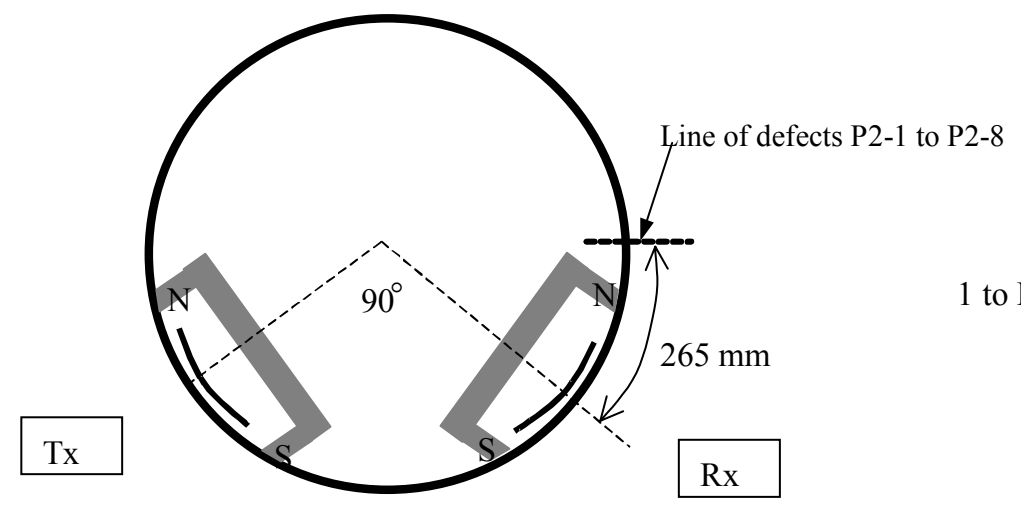

Figure 18 Schematic of the 'reflection' scans. Magnets set for SV1 mode biasing.

The set-up for transmission scans is shown schematically in Figure 19. The defect is clockwise from the receiver between it and the transmitter. In this arrangement the receiver EMAT head $(R x)$ will receive the direct signal from the transmitter $(T x)$ after it has interacted with the line of defects and travelled counter-clockwise 90 degrees. The next signal received will be the backpropagated wave from the transmitter (travelling clockwise 270 degrees around the pipe circumference) which will not have traversed any defects. This back-propagated wave may therefore be used to normalize the direct arrival in order to compensate any transmission loss and coupling effects in the direct signal. 


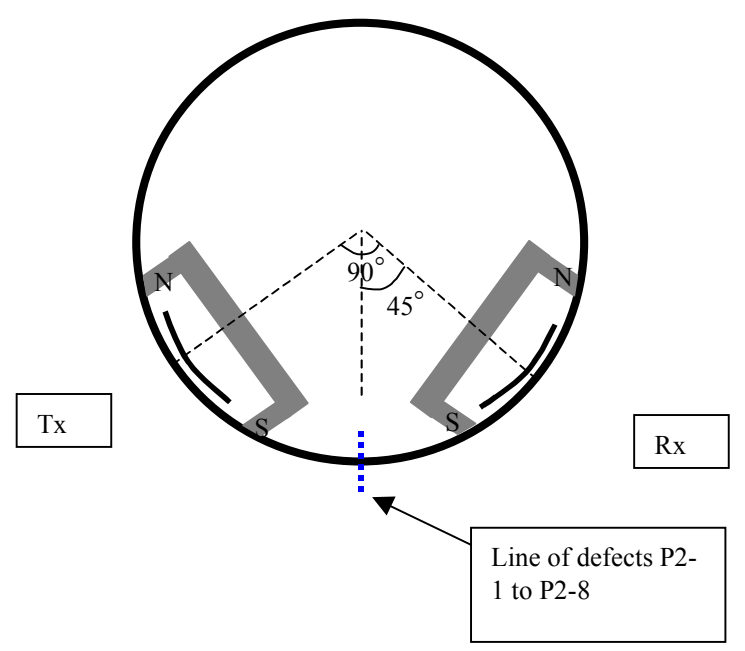

Figure 19 Schematic of the 'transmission' experimental set-up used to scan pipe sample P2

For mouse scan data shown in the Results section the previous arrival components were at fixed times, independent of axial position of the EMATs. This was because the defects were in a line, the mouse orientation was fixed, and waveform speeds were constant. Because of this, for a given mode (SH0 or SV1) all the various arrival amplitudes were obtained as peak to peak values in each of their own fixed windows. These values were then plotted versus EMAT axial position in various scan plots.

The previous window time positions were set by the group speed for the mode being scanned and the distance involved. For example, for the $\mathrm{SHO}$ defect reflection, the window time center were given by:

(Distance from transmitter to defect + Distance from the defect to the receiver) / SH0 group speed

The window width was usually two or three times the number of transmitter drive cycles and was not critical.

\section{Mule and Prototype Tool Developments and Evaluations}

\section{Components}

The philosophy behind the mule developments was to shrink the subsystems in Figures 6 and 16 and reduce power requirements, so that these components could be mounted on a pull-able mule and later used for a prototype tool without much modification. Large reductions in size would be needed. Specific ideas are diagrammed in Figure 20:

Magnetic biasing was done by permanent magnets instead of electromagnets in the mouse. This saved considerable power, as well as the space for large DC bias supplies. The magnets and transducers could be mounted on modules or sleds that would allow for substantial gas bypass and collapse. Thus, the transducer deployment would allow for slow logging without interfering with gas supply. It also would allow for obstructions, diameter changes, and sharp bends. The EMATs were two layer meander line coils (4 periods, $60 \mathrm{~mm}$ by $50 \mathrm{~mm}$ ), which doubled the signal level and were easier to impedance match than single layer.

For the control, a field programmable gate array (FPGA) plus high frequency crystal clock chip replaced the signal generator and allowed flexibility for frequency and drive specifics. For the 
transmitter driver, a pulse (Class D) amplifier increased output power, efficiency and reduced the design to a few chips and discrete power transistors. The output circuits were inherently low impedance $(\sim 1 \mathrm{ohm})$, as provided by power MOSFETs, and so were directly matched to the low impedance of the EMATs and eliminated the need for resonant matching networks. Thus, space was saved and different frequencies for SHO and SV1 were easily accommodated by means of the logic of the FPGA. Receiver matching was done by broadband transformers to accommodate the different SHO and SV1 frequencies and also to take advantage of available low noise operational amps. For the transmitter, compact high voltage power supply modules were used that supplied symmetrical positive and negative voltages to simplify drive circuits.

In an ILI tool the previous components would be placed near their respective transducers, outboard of the primary pressure capsule. Moderate level (a few volts at low impedance levels) receiver and transmitter control signals and battery voltage would pass between the primary pressure capsule and out board transducer assemblies. This approach would result in minimized transmitter loses, firing cross-talk, noise pickup, connections between the primary capsule and the transducers. It would be safer than having high voltages going between the primary capsule and the transducers. The digitizer, data reduction/defect extraction, memory store, and distance measuring systems would be in the primary capsule with the main memory.

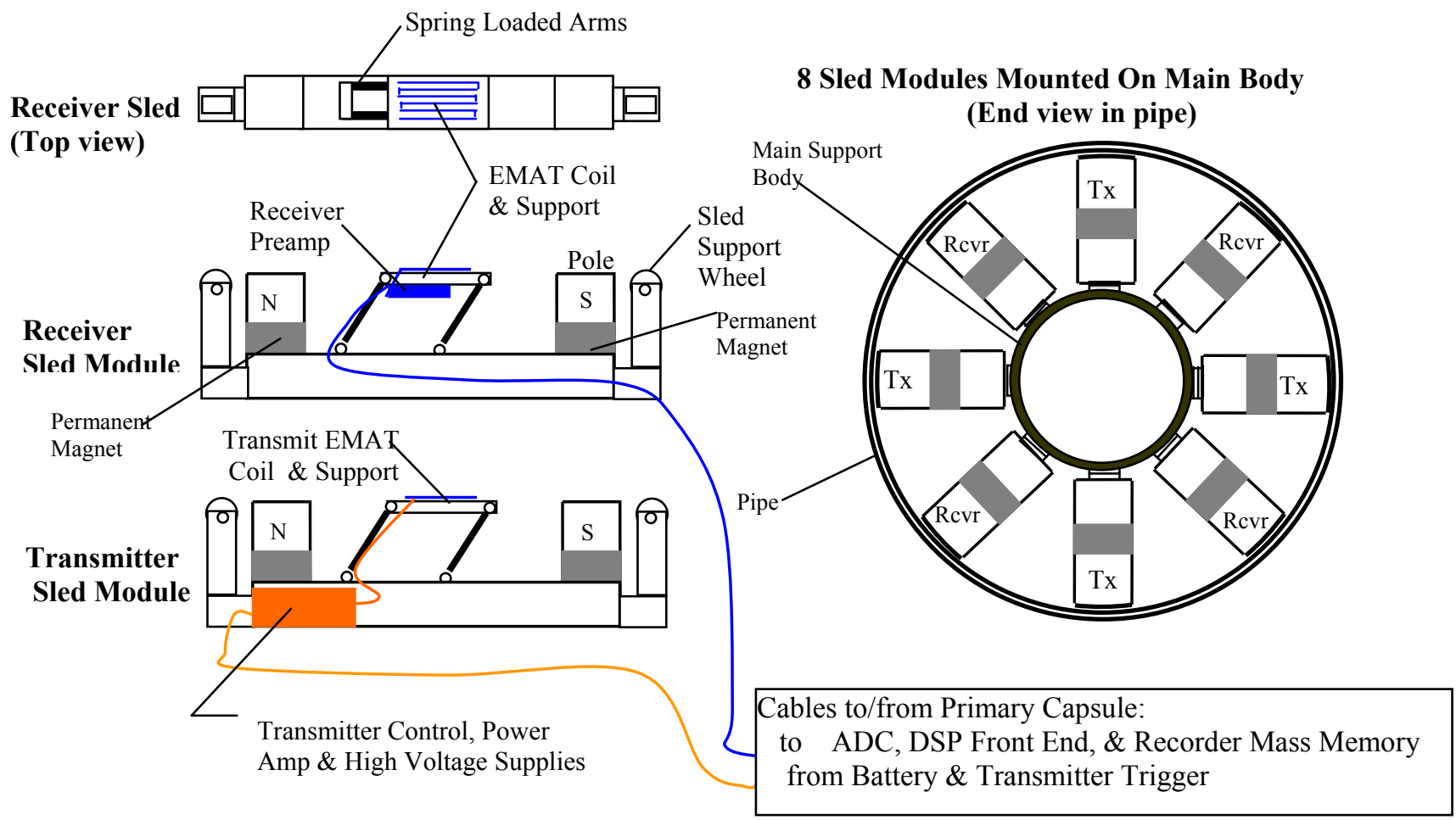

Figure 20 Illustration showing sled modules in an SHO configuration and some associated acquisition blocks for an ILI or mule system. Note in the end view that transmitter (marked TX) and receiver (marked Rcvr) sleds alternate. Note also that support details have been omitted in some views for clarity.

Compact sled modules were built that can be mounted every 45 degrees on a mule and so provide an axial magnetic bias field for SHO EMATs. Free field measurements and simulations showed that the magnetic field in targeted pipe walls was at or close to saturation (i.e. close to 2 
Tesla). Transmitter and receiver coils would be mounted between the poles on alternate modules as in Figure 20.

Prototype mule circuits, compact enough to fit on sled modules or behind ML coils of a mule and prototype ILI tool, are shown in Figures 21 and 22). These worked with a digitizing scope to acquire and store waveforms, but in an ILI tool they would work with an ADC and recorder-mass storage system. The transmitter and high voltage power supplies (Figure 21) were stacked into a unit that is $\sim 6 \times 4 \times 2$ inches on three cards The power supplies converted the battery to a $100 \mathrm{~V} \mathrm{DC}$ and had enough storage to sustain high peak currents. The transmitter was a high power pulser driven by a single control FPGA. It allowed variation of center frequency, number of cycles, firing repetition rate. It also randomly jittered the firing rate, so as to minimize rep rate noise (echoes) in conjunction with signal averaging.

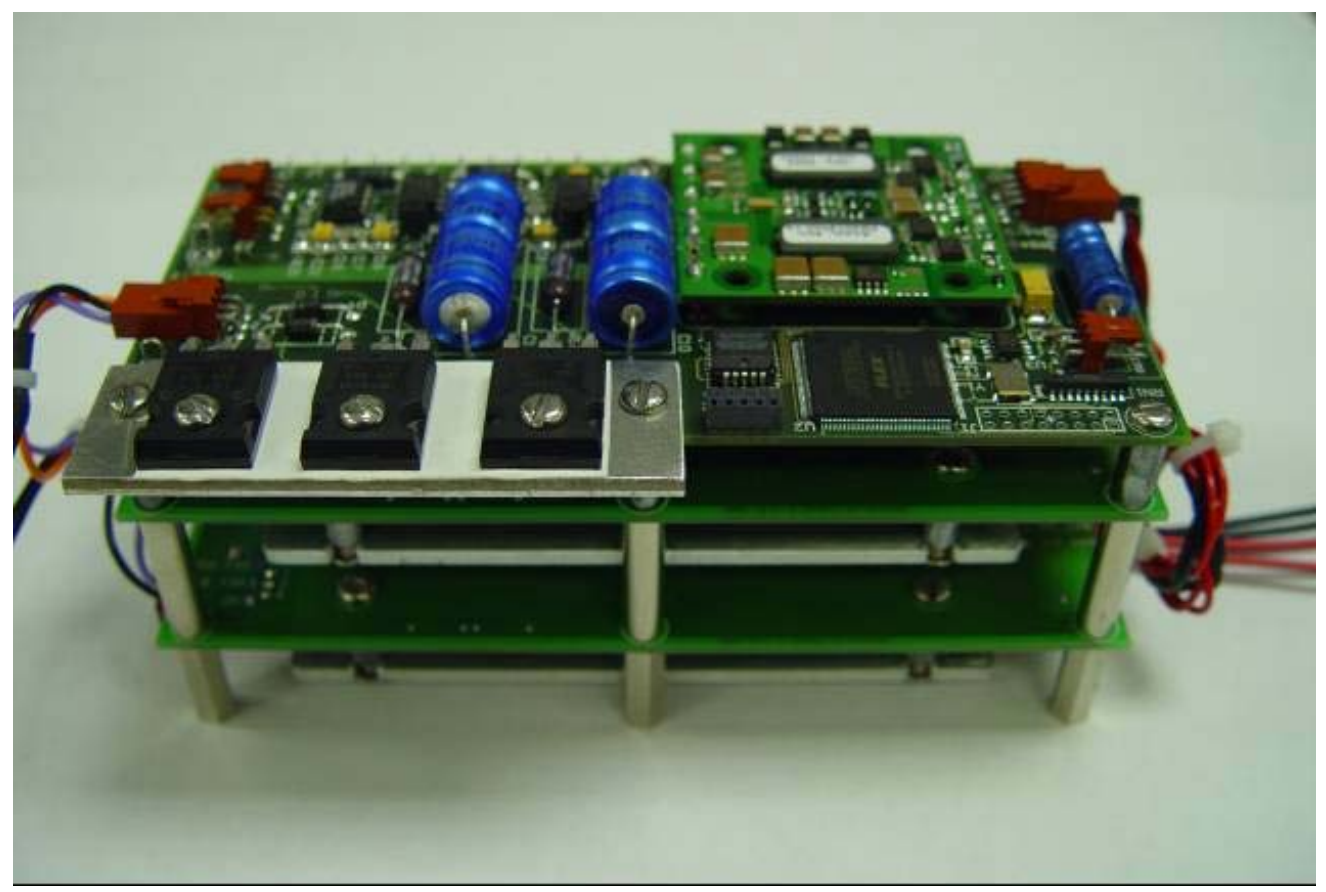

Figure 21 Mule prototype transmitter control (top board), driving stacked above positive and negative high voltage supply boards

Figure 22 shows the preamp. This is $4 \times 3 \times .5$ inches, so it could fit behind the EMAT to minimize lead lengths and noise pick up. The preamp circuit consisted of a broadband (ferrite core) transformer for impedance matching followed by a very low noise AC amplifier, other low noise amplifiers, and a high pass and then a low pass filter - each 2 pole. It also included voltage regulators.

Prototype permanent magnet EMAT transducers were made for the mule for evaluation with the mule electronics. These consisted of two groups of bar magnets as legs supporting a soft steel back iron with an EMAT ML coil between the magnets. The field in a .0625" air gap under the magnet was $\sim 4000$ Gauss. As shown in Figure 23, the EMATs were on the outer pipe surface for SCC grading, but they were also used for various signal quality measurements on the inner pipe surface and on flat plates. 


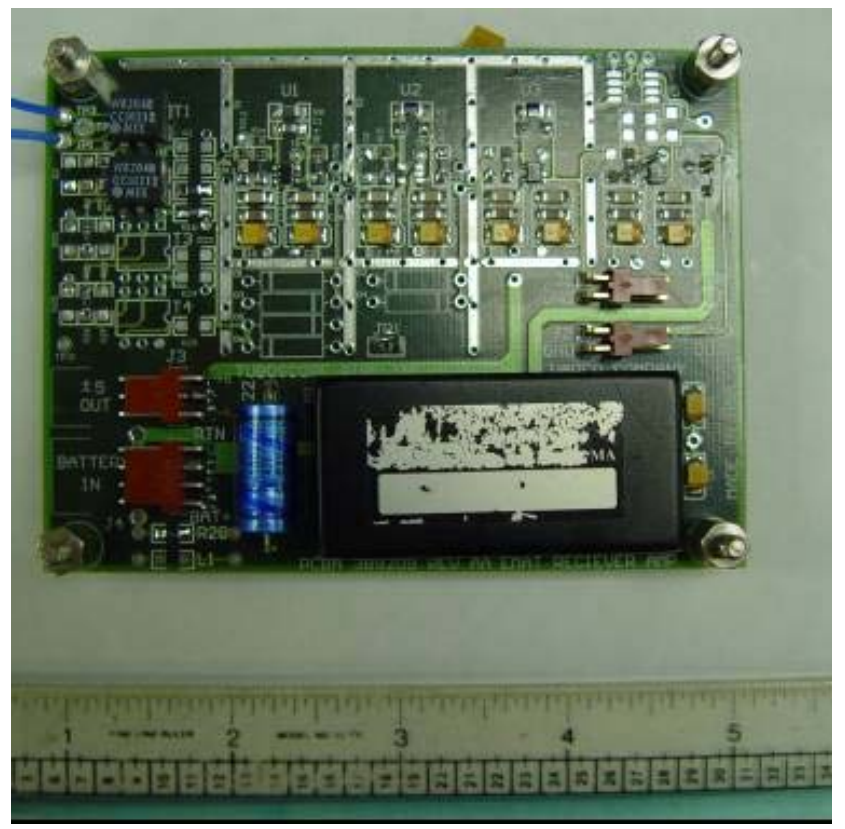

Figure 22 Mule prototype receiver preamp board with input matching transformer pair and regulators

Evaluations of Mule Components (Velocities, Attenuations, Man-made SCC)

To evaluate signal quality, dispersion, coupling versus materials, power levels, and other issues for the mule prototype components, flat plate and full pipe tests were made with a pitch catch setup for plates of different thickness and materials and with permanent magnet biasing. In these tests the direct arrival versus transmitter to receiver separation was monitored. The parameters examined were peak to peak amplitude, envelope peak amplitude, phase, and time of the peak in envelope. Phase velocity was obtained from the time difference between the zero crossings of the 5th cycle for direct arrivals at two separations, usually 8 and 15 inches. Group velocity was obtained from the time difference between the peaks in envelopes of direct arrivals at two separations.

In order to have SCC-like defects, slits with known lengths (along the pipe axis) and depths were made by EDM on the outside of the pipe. The slits were $0.2 \mathrm{~mm}$ wide along the pipe circumference. The pattern and position of these defects is shown in Figure 24 along with positions for the transmitter and receiver for a reflection scan. The depths, lengths, and widths were verified by means of actual casts made in the slits. The main reasons for testing with such slits is that the SCC tested by the mouse (Figure 17) was too shallow (<25\% wall thickness), was not known explicitly, and could not be measured by another means. The reflection scans were done similarly to those for the mouse (Figure 18), except the angle between transmitter and receiver was 45 degrees.

For all the mule evaluations, receiver waveforms were digitized at $12.5 \mathrm{MHz}$ for intervals of 1 to $2 \mathrm{~ms}$. The converter resolution was 8 bits. The interval was long enough to capture arrivals making one or two trips around the 30" pipe or back and forth in the plate. For the prototype component evaluations it was sufficient to use waveform envelopes. These were extracted digitally in bandwidth of $\sim 100 \mathrm{kHz}$ and resampled at as low as .5 $\mathrm{MHz}$. The envelope extraction, illustrated in Figure 25 for a close-up of a SHO direct arrival, shows that amplitude information is retained 
Mule transmitter power amp, controller, \& high voltage supplies

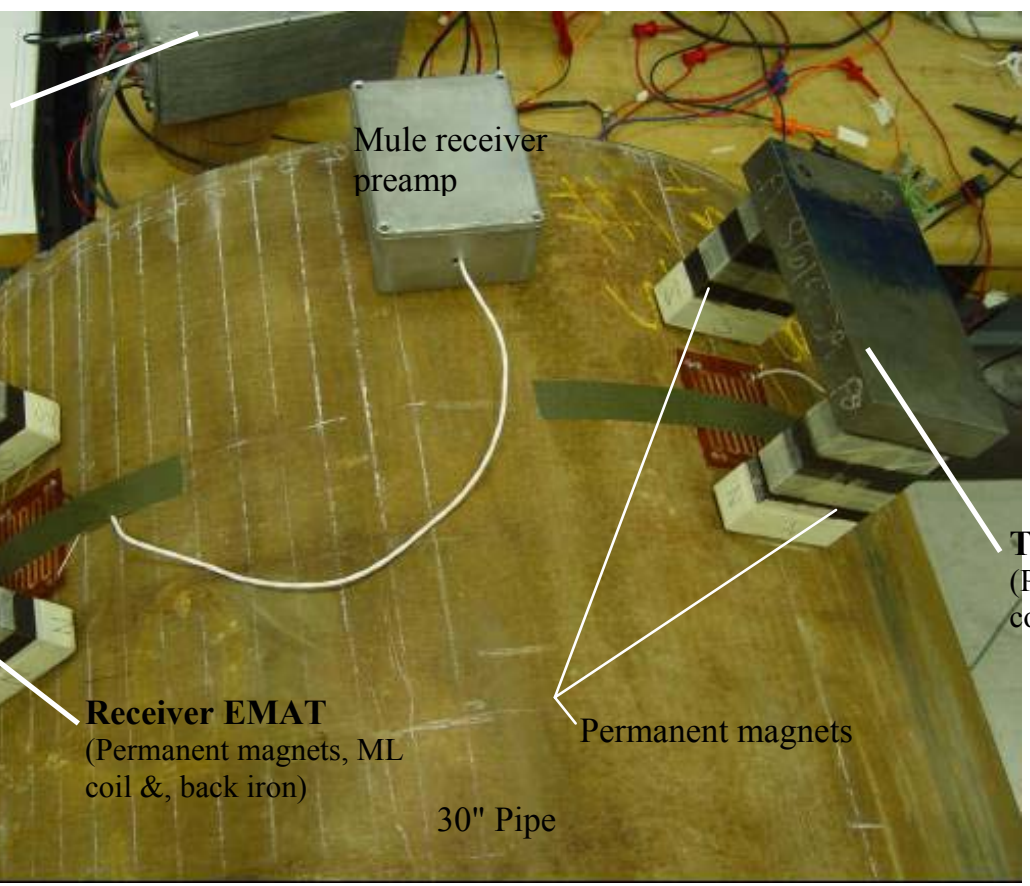

Transmitter EMAT

Permanent magnets, ML coil \&, back iron)

Figure 23 Mule prototype evaluation and test setup, showing permanent magnet EMATs configured for SHO measurements on 30" pipe with man-made SCC.

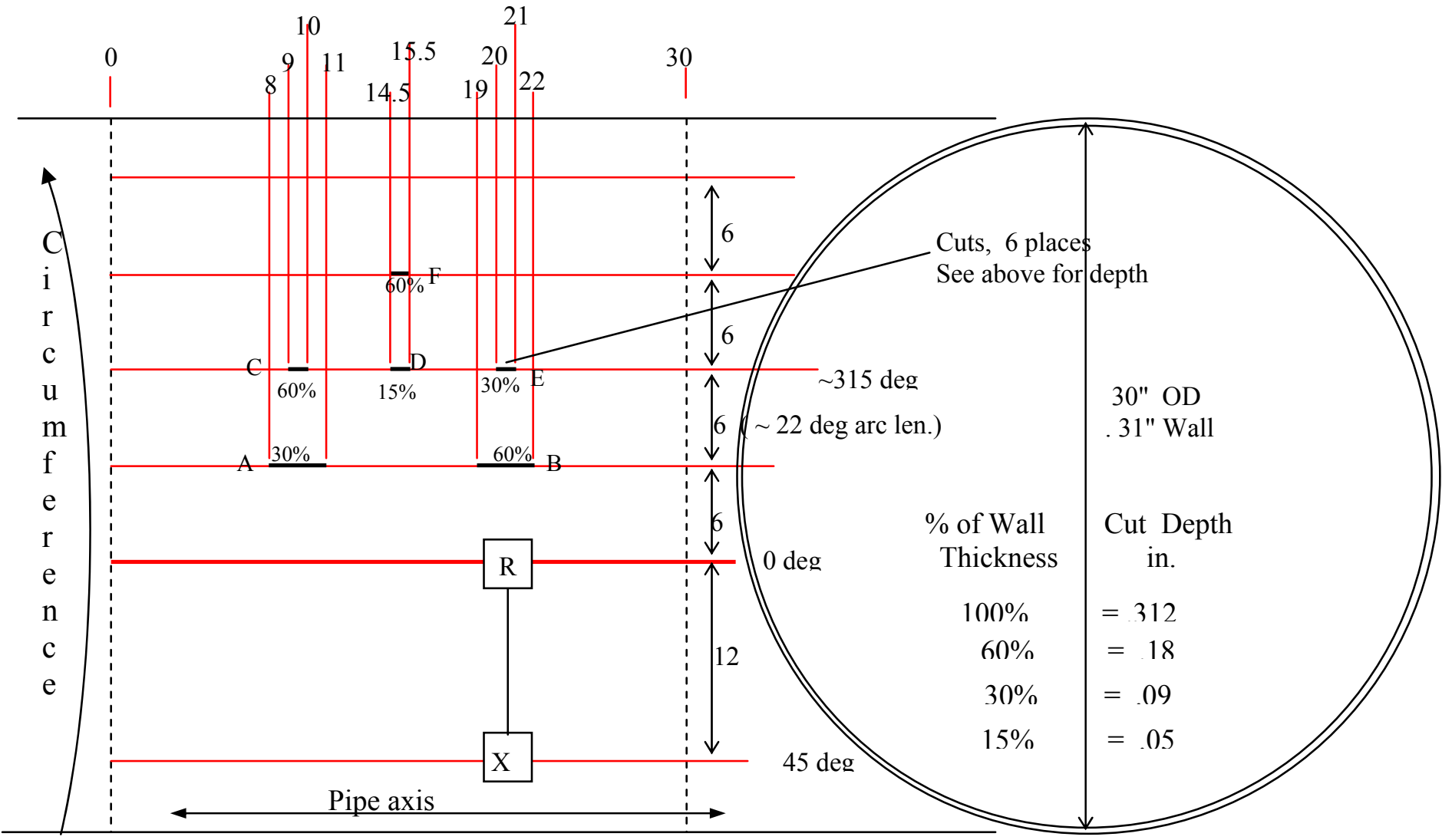

Figure 24 Pattern of man-made SCC cut defects on 30" pipe for prototype mule tests 


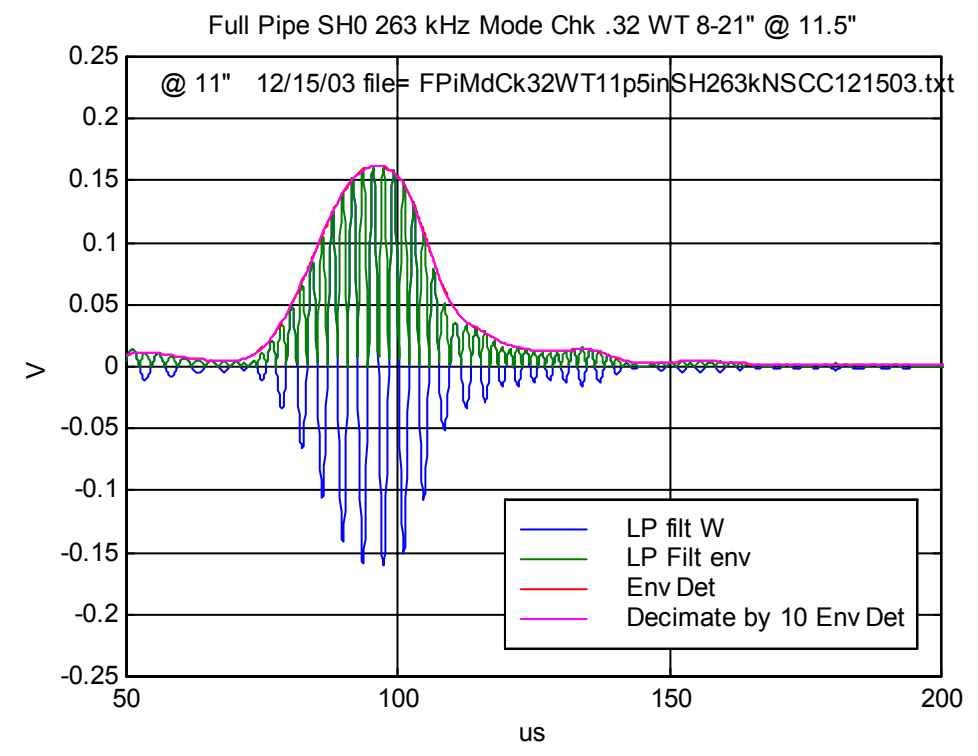

Figure 25 Envelope extraction and data reduction for a direct arrival at 11" from transmitter to receiver in 30" pipe. One of every 10 samples of the curve, "Decim by 10 Env Det", which ovelays Env Det.

Figure 26 shows a typical waveform envelope for a pipe scan measurement taken with the setup of Figure 23 for $\mathrm{SHO}$. The various arrivals are marked on the figure. It illustrates all the waveform arrivals in a pipe scan. The firing transient, which normally saturates the digitizer, has been zeroed out, and this waveform has also been normalized, so that the direct arrival is near unity.

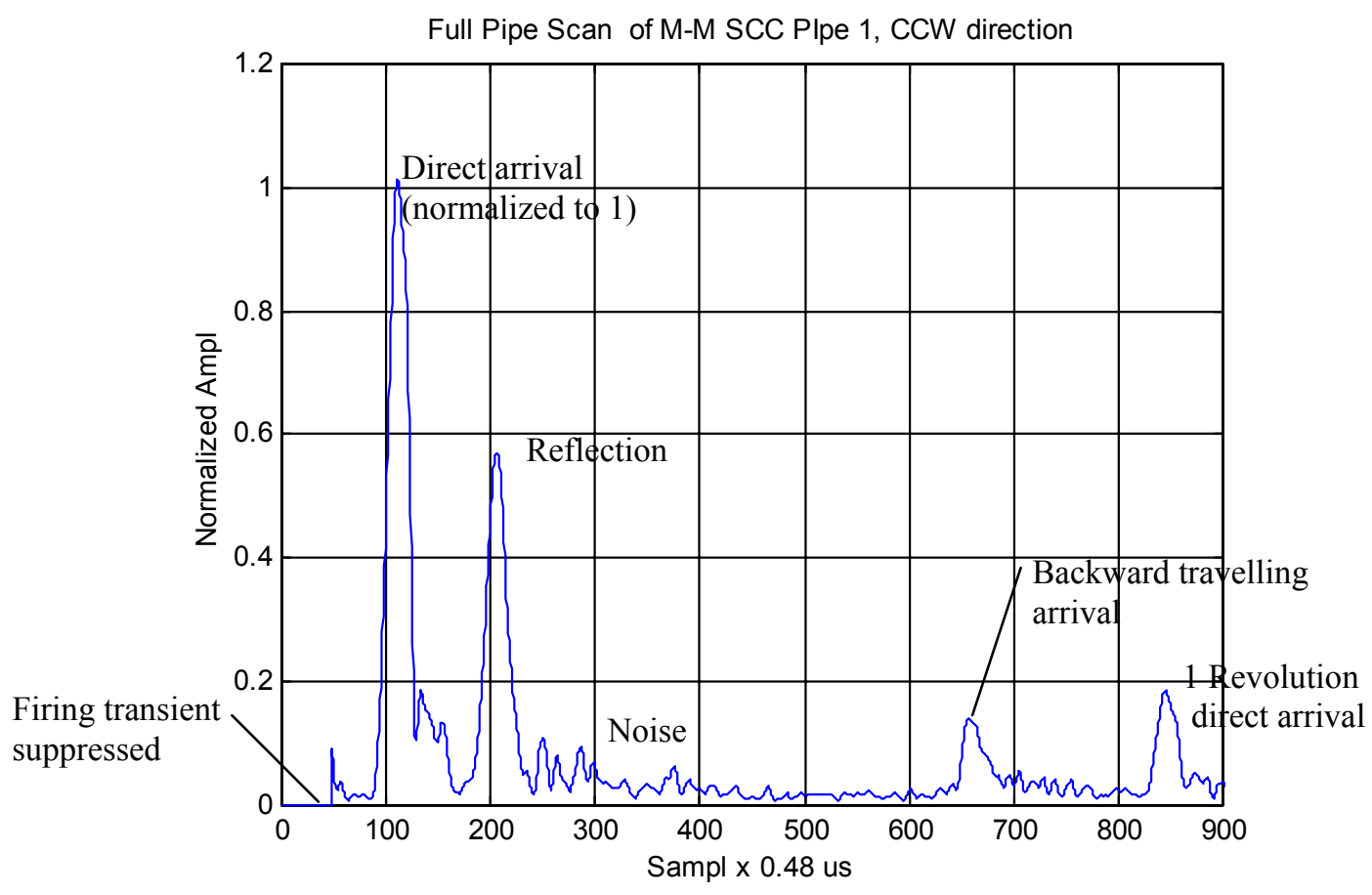

Figure 26 Typical full pipe normalized (for unity direct arrival amplitude) waveform envelope obtained by mule prototype circuits and permanent magnet SHO biased EMATs. 
As with mouse data, the arrivals for mule data occurred at fixed times and independent of axial the position of the EMATs. Thus, as with the mouse all the various arrival amplitudes were obtained as peak to peak values in each of their own fixed windows. These values were then plotted versus EMAT axial position in various scan $(A$ or $C)$ plots.

Attenuation measurements were made on a 30 " diameter, $8 \mathrm{~mm}$ thick pipe on a spot without defects. This was done for three conditions: uncoated, one layer of coating and two layers of coating. The coating was Densopol $60^{1}$ heavy duty PVC/Bitumen fabric reinforced tape. Figure 27 shows the pipe with one coating layer. The transducers were fixed inside the pipe in the middle of the coating stripe for each condition. Attenuation was based on the ratio of the backward arrival peak to the direct arrival peak. (Refer to Figure 26 for the arrivals.) over the difference of the backward and direct travel paths. Linear attenuation ( $\mathrm{dB} / \mathrm{inch})$ is then given by:

\section{Linear Attenuation =}

\section{0*LOG10 [(Backward Arrival Peak/ Direct Arrival Peak)/(Backward Path -Direct Path) ]}

Use of the ratio of backward to direct is very beneficial since it eliminates any disturbing effects of transducer coupling or standoff from the attenuation measurement because amplitudes at two distances are obtained with the same receiver and transmitter and without moving them. For each condition attenuation measurements were made at 16 and 22 inch transmitter to receiver separations and averaged.

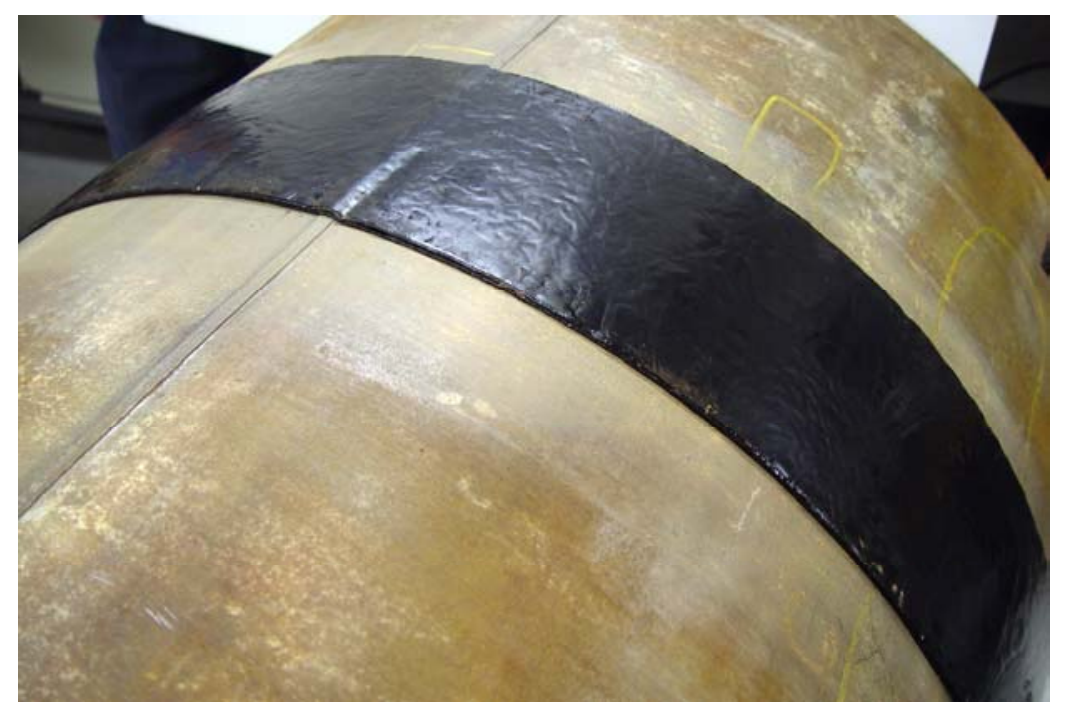

Figure 27 Pipe (30" diameter, $8 \mathrm{~mm}$ wall thickness) with a single layer coating used for attenuation measurements. The EMAT transducers were located inside the pipe and were centered under the coating band. Each coating layer material was Densopol 60, $1.6 \mathrm{~mm}$ thick coating tape from Denso North America.

\footnotetext{
${ }^{1}$ Made by Denso North America, 18211 Chisholm Trail, Houston, TX, USA 77060 


\section{Results and Discussion}

\section{Best Modes and Transduction Type To Pursue}

Initially, in the lab we tried both Lorentz and magnetostrictive transduction. Although signal to noise ratios and power were similar for both, we decided to pursue magnetostrictive, because it had additional advantages. First, magnetostrictive allows the bias magnets to be separated from the EMAT coil. Thus, a light ML coil could be sprung without a heavy load on it, and the bias magnets, surrounding the coil, could even provide some protection. This is a much more rugged situation than occurring for a Lorentz EMAT, which must have the bias magnet above the coil. Another advantage of the magnetostrictive is that the bias field required is in the plane of the pipe, and so it is easier to establish than the perpendicular field required by a Lorentz EMAT. The Lorentz magnet arrangement (Figure 2) will be also larger radially than the magnetostrictive, making it more expensive and less able to accommodate diameter restrictions. Finally in flat plate tests, we observed more complicated waveforms and more dispersion for Lorentz transducers, which indicated less modal purity for Lorentz. This was probably due to the fact that its bias field was diverging from perpendicular.

Because it would be impractical to implement all six of the candidate modes in Table 1, we narrowed the list to the SV1 mode at $470 \mathrm{kHz}$ and the SH0 mode at $260 \mathrm{KHz}$. These were picked because of ease of implementation, limited size of the EMAT head, low sensitivity to standoff and tilt, low expected attenuation, good modal purity and minimal interference from other modes. As described in the first annual report [13], the other modes were easily eliminated due to one or more major failings.

Two modes were chosen to pursue (through the lab mouse tests) in case one choice led to a blind alley and because they had complimentary crack sensitivities. SV1 was sensitive to shallow cracks, while SHO was sensitive to deep cracks. Both had good to excellent SNRs. The $\mathrm{SHO}$ had a lower signal to noise ratio and required extra power. However, SHO had the theoretic advantages of no dispersion, less attenuation (due to pipeline coatings) and of simpler crack interactions ${ }^{2}$. We could not eliminate either mode because in all other factors they were similar. For example, both had nearly the same wavelength. Later, as described in Additional Measurements With Mule Components, we found that SV1 had too much dispersion, so we dropped SV1.

\section{$\underline{\text { Lab Improvements and Transducer Measurements }}$}

Improvements, SNR, Drive Current, and Insertion Loss

To save space in this section we concentrate on the previously mentioned chosen transduction type and modes. We found that good matching circuits and complete shielding was absolutely necessary for good signal to noise ratio, efficiency, and recovery from the firing transient. We also noted that if the resistance value gets too low, the $Q$ of the matching circuit must be very high which makes circuit stability and ruggedness hard to achieve. Additionally, the acoustic signal will be proportional to the number of turns in the EMAT coil. Consequently for optimum signal output there will be a trade off between the number of turns and the resistance of the coil for optimum coupling (and hence drive current). In our work we found this was achieved for $\mathrm{SHO}$ and SV1 with two layer coils with a resistance of 1 to 2 ohms. The two layer coils doubled the SNR obtained from one layer and made the matching circuits less critical to implement.

\footnotetext{
${ }^{2}$ This because SH modes should not convert to other modes at boundaries between materials.
} 
With the improved flat plate set-up the sensitivity of the receive EMAT coils was significantly increased. This was especially so for the SV1 $3.91 \mathrm{MHz}-\mathrm{mm}$ mode in which a "live" (unaveraged) signal to noise ratio (SNR) of $\sim 100: 1$ has been achieved using a single-layer transmit coil and a drive current for the transmit coil was measured at 25 Amps (peak). The insertion loss for this arrangement was calculated to be $\sim 120 \mathrm{~dB}$. SNR for the SH0 EMAT receive heads was initially less than 1 , with the result that $\mathrm{SH} 0$ could hardly be observed. After improvements, the live SNR ratio of $\sim 30$ to 1 was achieved with a drive current of 22 Amps (peak). An example trace of the $\mathrm{SHO}$ mode at $2.14 \mathrm{MHz}-\mathrm{mm}$ is shown in Figure 28 . The insertion loss was about $130 \mathrm{db}$.

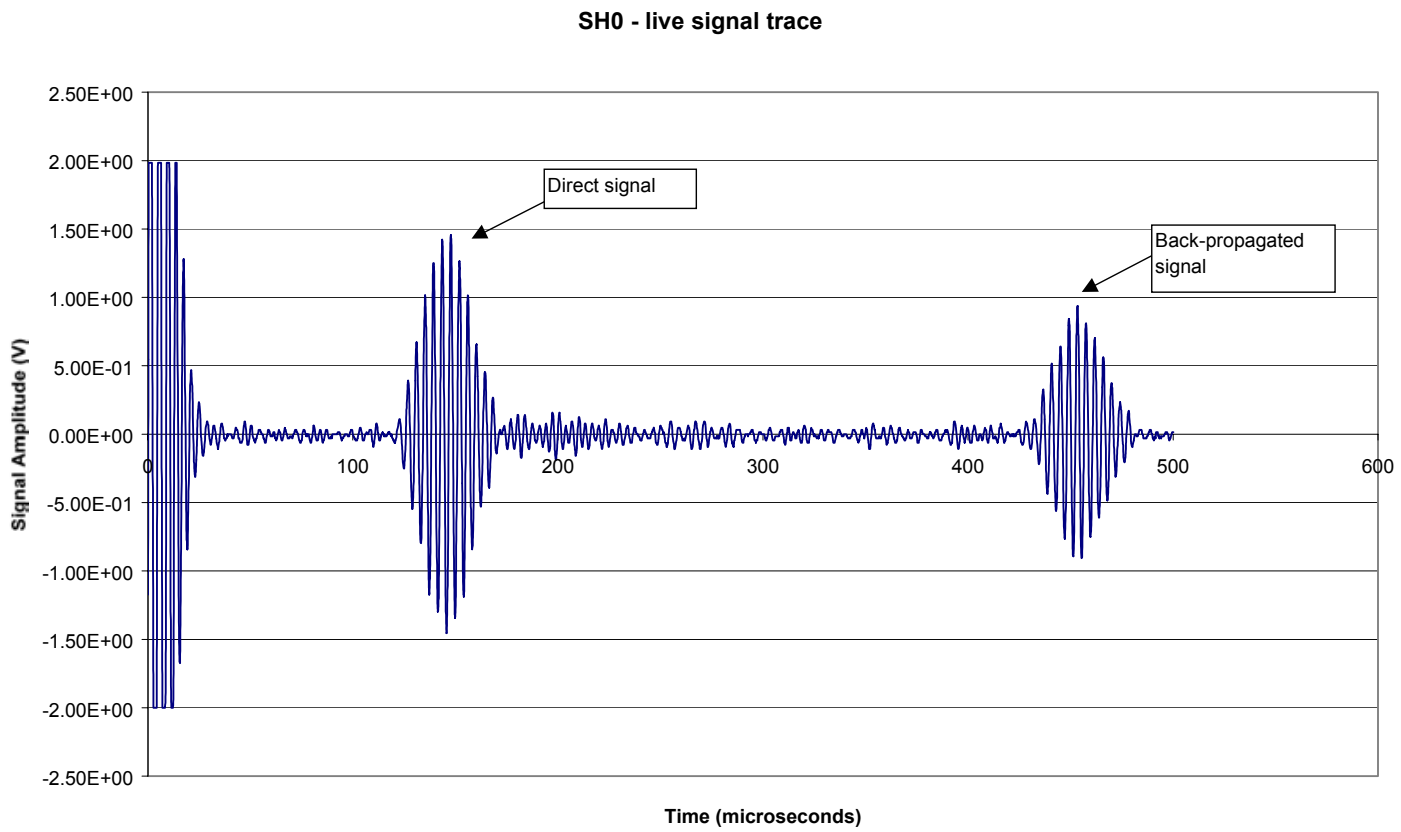

Figure 28 Example live trace of the received signal from an SHO toneburst with the SHO 'mouse' set-up

All of the work described above was carried out using meander line (ML) EMAT coils of the type and size shown in Figure 8. It was thought that for the SH modes, increased sensitivity and improved insertion losses might be achieved through using pancake EMAT coils with periodic permanent magnets (ppm heads). Some work was carried out on ppm-SH heads. No significant improvement to insertion losses was apparent, and because these heads had a large and endangering magnet mass behind their coil, we dropped further development.

Beam Width

Figures 29 and 30, corresponding to the beam width measuring geometries of Figures 10 and 11 , indicate that for this particular geometry of EMAT coil, very little beam divergence occurs to propagation distances of at least $500 \mathrm{~mm}$. Figure 30 also indicates a regular shaped angular response without side lobes at 90 degrees to create confusing artefacts. Moreover, if we take the beam width to be within points where the amplitude is decreased by .3 from the peak at the beam center, we can see that the beam width is $+-4 \mathrm{~cm}$ or $80 \mathrm{~mm}$ wide - about the width of the 
EMAT ML coil. Without additional techniques, such as synthetic aperture digital processing, this is the axial resolution.

Beam profile from EMAT head

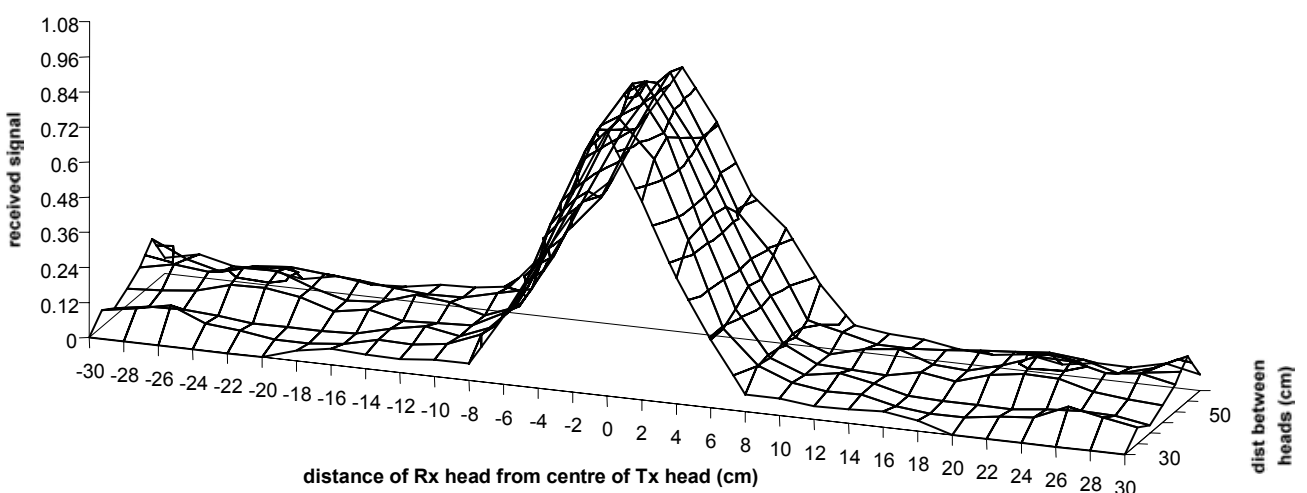

Figure 29 Beam profile of the SV1 EMAT transmit coil (80mm wide)

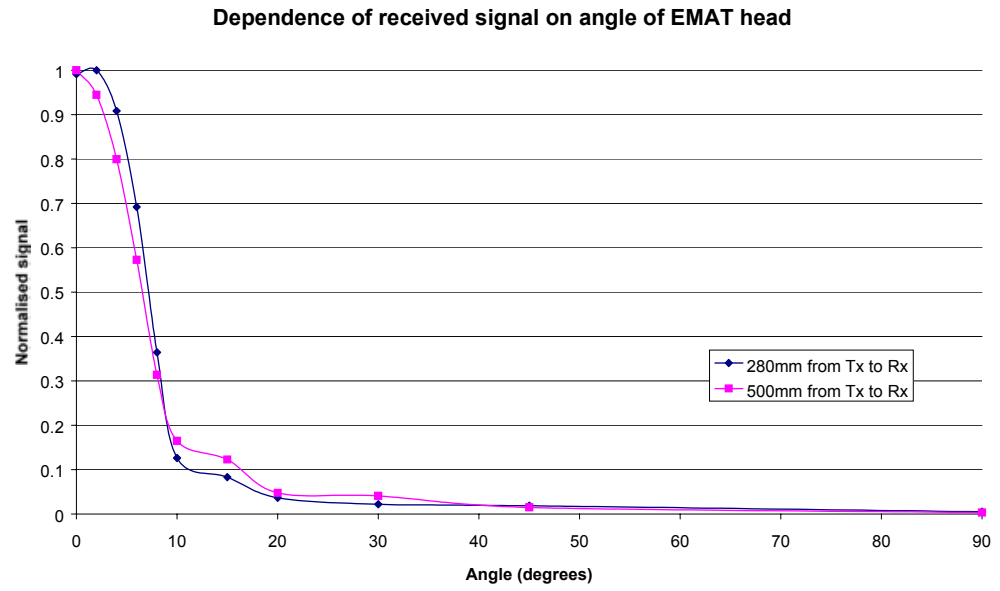

Figure 30 Angular dependency of SV1 mode

Effect of Bias Field

The results using a bias field sweep through both positive and negative field values are given for the SV1 mode (3.91 MHz-mm) in Figures 31 and 32. These plot received signal amplitude (vertical axis) versus bias electro-magnet current, which is effectively the magnetic bias field. Note that the bias current from one transducer was swept while the other was held at a fixed value. Note also that portions of sweeps, referred to here as "quadrants" that have different histories (i.e. current increasing, decreasing, or at a later time) are shown in different colors. 
The results for similar bias level sweeps, for the SH0 mode (2.14 MHz-mm), are shown in Figure 33 and 34. These figures indicate that for transmitter and receiver EMAT coils and for either SV1 and SH0 modes 1) signal level is proportional to bias level and 2) the maximum signal occurs for magnetic saturation.

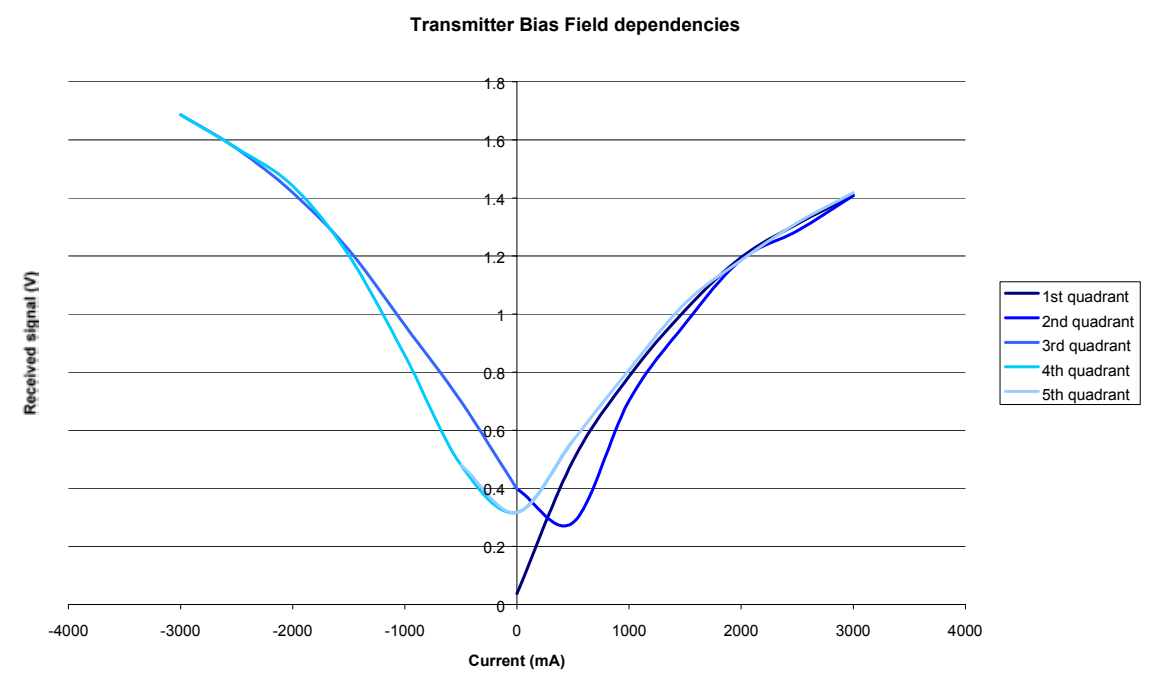

Figure 31 Dependency of the signal amplitude for the SV1 mode on the bias field of the transmit EMAT coil

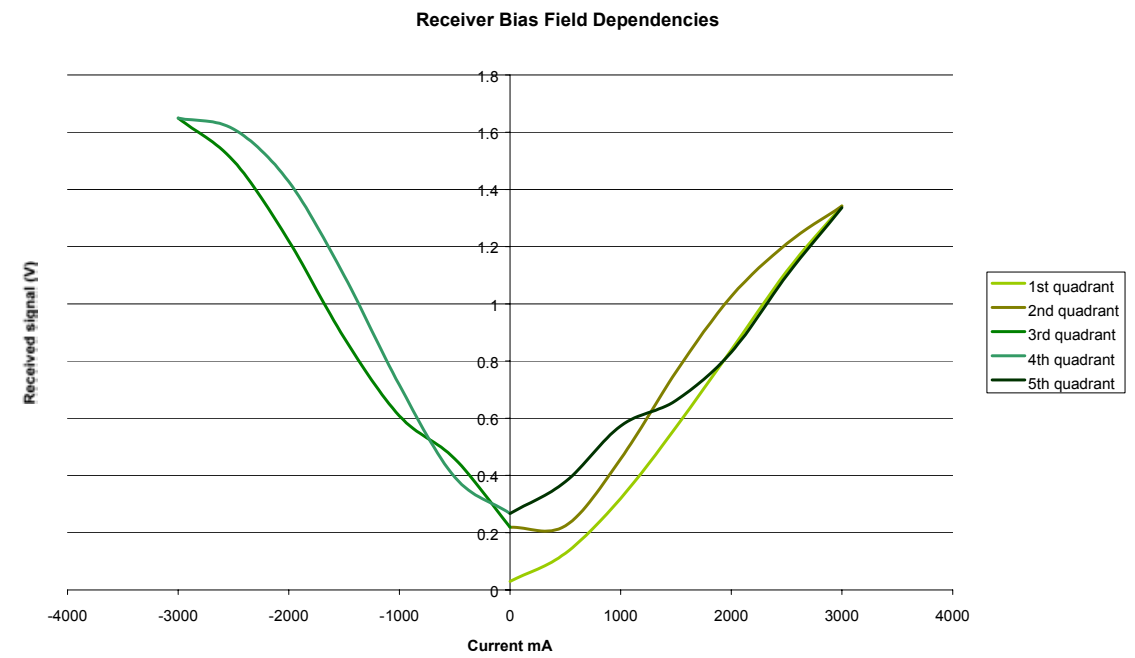

Figure 32 Dependency of the signal amplitude for the SV1 mode on the bias field of the receiver EMAT coil. 


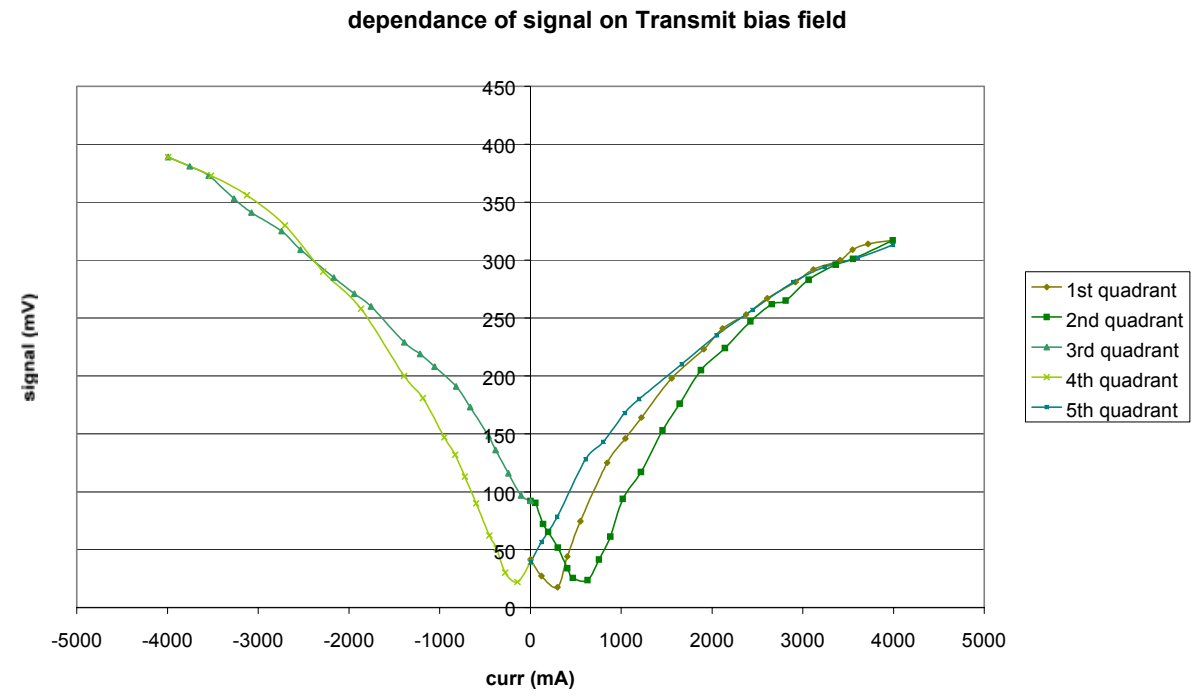

Figure 33 Dependency of the signal amplitude for the SHO mode on the bias field of the transmitter EMAT coil.

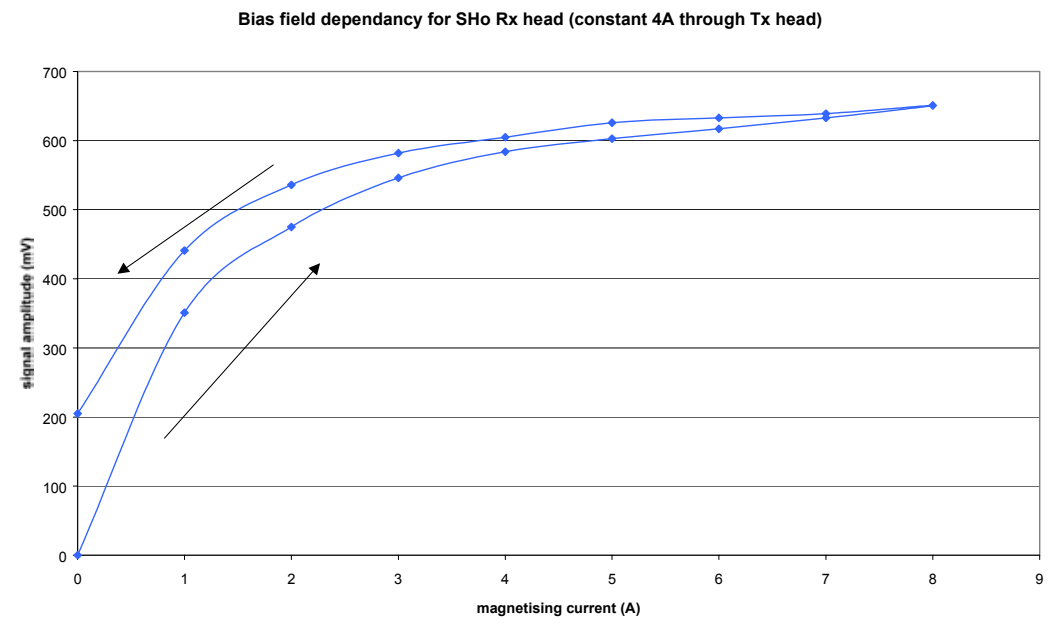

Figure 34 Dependency of the signal amplitude for the SHO mode on the bias field of the receiver EMAT coil at high bias currents.

The SV1 and SH0 signal level dependencies on magnetic bias were investigated again in a 24 inch diameter full pipe as a check against the previous results in a plate or pipe sections. This was done with the transducers separated as if they were in a useful configuration. The reason for this check was that the transducer bias magnets would be close enough to interact in the closed pipe. The conclusions were the same as those of the plate.measurements.

Sensitivity to standoff and tilt

The effect of stand-off is shown in Figure 35: for the receiver in blue and the transmitter in green. A value of 1 represents a normalized amplitude with minimal standoff. For a meander line (ML) coil, the signal amplitude is expected to follow $\exp (-c G / D)$, where $G$ is the coil standoff from the surface, $D$ is the interline spacing for the meander coil, and $c$ is a positive 
constant [8]. A good fit to the data was obtained for $y=1.08 \mathrm{e}^{-0.67 x}$. Thus, standoff sensitivity is as expected and decays exponentially with standoff.

If the EMAT is designed properly, $D$ is half the wavelength. Thus, amplitude versus standoff should depend on wavelength (i.e. D) and decays exponentially with the ratio of standoff to wavelength. By this wavelength reasoning, the results for the $\mathrm{SHO}$ (at $260 \mathrm{KHz}$ ) EMAT coil will be identical to those of SV1 since its wavelength is nearly the same and the coil geometries are the same ${ }^{3}$. Also, shorter wavelengths will have smaller $D$, larger $G / D$, and hence larger (exponential) amplitude loss with standoff. Because of this use of shorter wavelengths will allow less standoff in absolute terms and will be more sensitive to standoff. Note that we believe we can tolerate an amplitude reduction to .3. This corresponds to a standoff of up to $2 \mathrm{~mm}$ for SV1 $(470 \mathrm{kHz})$ and $\mathrm{SHO}(260 \mathrm{kHz})$.

The dependence of the received signal amplitude on tilt angle is shown in Figure 36 . Front to back tilt, is shown is pink/purple, and sideways is shown in blue. It can be seen that for this coil design the signal is most sensitive to 'sideways' tilt (with respect to the propagation direction) as compared to 'front-to-back' tilt. Note that on an ILI pig, front-back is really circumferential and sideways is really axial for a magnetostrictive EMAT. Note also that the amplitude variation for standoff and tilt in Figures 35 and 36 are nearly identical for transmitter or receiver, as expected because the transmitter and receiver EMATs should be reciprocal and because they have the same geometry and biasing.

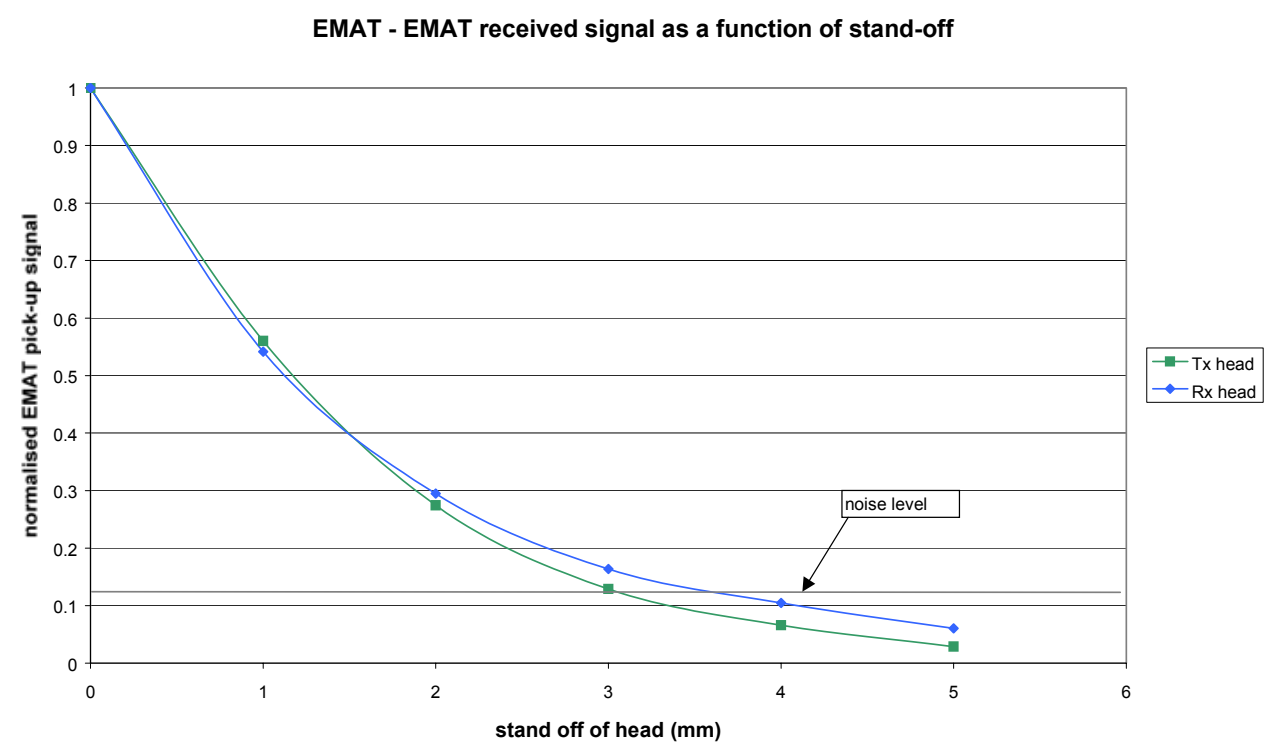

Figure 35 Effect of stand-off on the signal amplitude for EMAT-to-EMAT; SV1 coil. (Note that standoff is actually $.1 \mathrm{~mm}$ larger marked. Thus, 0 standoff is actually $.1 \mathrm{~mm}$ )

\footnotetext{
${ }^{3}$ This was later verified with mule components for SH0 $(260 \mathrm{kHz})$. The results are omitted here to save space.
} 
Dependance of received signal on tilt of EMAT head

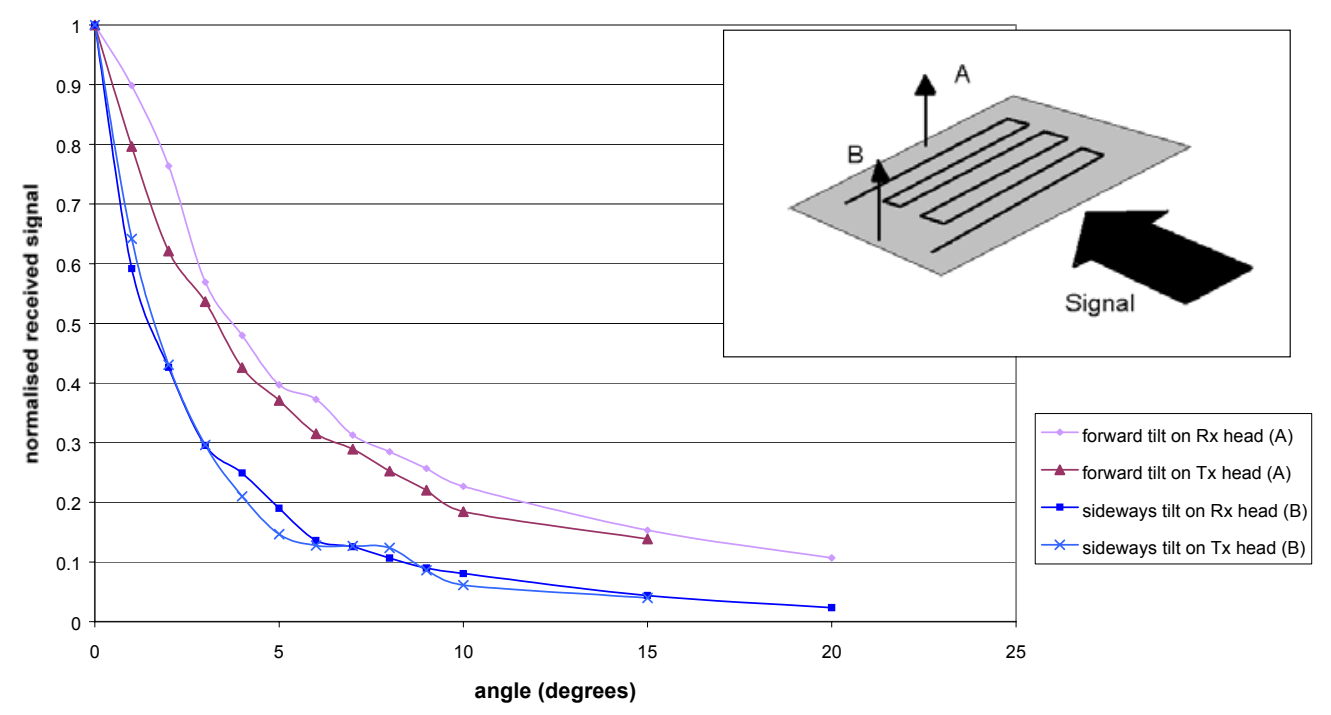

Figure 36 Effect of tilt on the signal amplitude for EMAT-to-EMAT; SV1 coil.

\section{$\underline{\text { Reflection and Transmission From Man-made Cracks (Measured and Modelled) }}$}

The reflection/transmission crack interaction results for the $8.4 \mathrm{~mm}$ thick flat plate are summarised in 37 and 38 , in which reflection coefficients are red, and transmission are blue. The modelled results are presented by dashed lines, while the interpolated, measured curves are solid. The modelled data was obtained by FEM, as described a report for GTI [12]. For both SV1 (at $3.9 \mathrm{MH}-\mathrm{mm}$ ) and $\mathrm{SHO}$ at $(2.1 \mathrm{MH}-\mathrm{mm})$, the modelled and measured coefficients exhibited similar trends versus crack depth and generally compare well up to about $40 \%$ crack depth. Also, there were significant effects from the cracks that were well above the noise level and easily detectable. For example, a crack depth of $20 \%$ has a $40 \%$ and $25 \%$ reflection for SV1 and SH0, respectively.

Note that the SV1 curves indicated a higher sensitivity for shallow crack depths than deeper ones. This is because the SV1 reflection and transmission curves flatten above $\sim 25 \%$ crack depth. For example, for a crack depth from $10 \%$ to $20 \%$ the SV1 reflection coefficient changes by .19 , but from a depth varying from $30 \%$ to $40 \%$, the SV1 reflection coefficient changes by only .10.

SH0 has a more uniform sensitivity to crack depth than SV1. For example, for crack depths from $10 \%$ to $20 \%$ and from $30 \%$ to $40 \%$ the SHO reflection coefficient changes by .13 . For shallow cracks this is less than that of SV1, so that it would be less useful in defining shallow cracks than SV1. However, for crack depths from $30 \%$ to $40 \%$, the SH0 coefficients show greater sensitivity to cracks, and so $\mathrm{SHO}$ would be more useful in defining deeper cracks than SV1.

Unfortunately, the measured $\mathrm{SHO}$ coefficients flatten above $40 \%$ depth, and we could not investigate above $50 \%$. Coefficients for cracks deeper than $50 \%$ need to be evaluated in the future. 
Transmission and reflection coefficients results for SV1 at $460 \mathrm{kHz}$

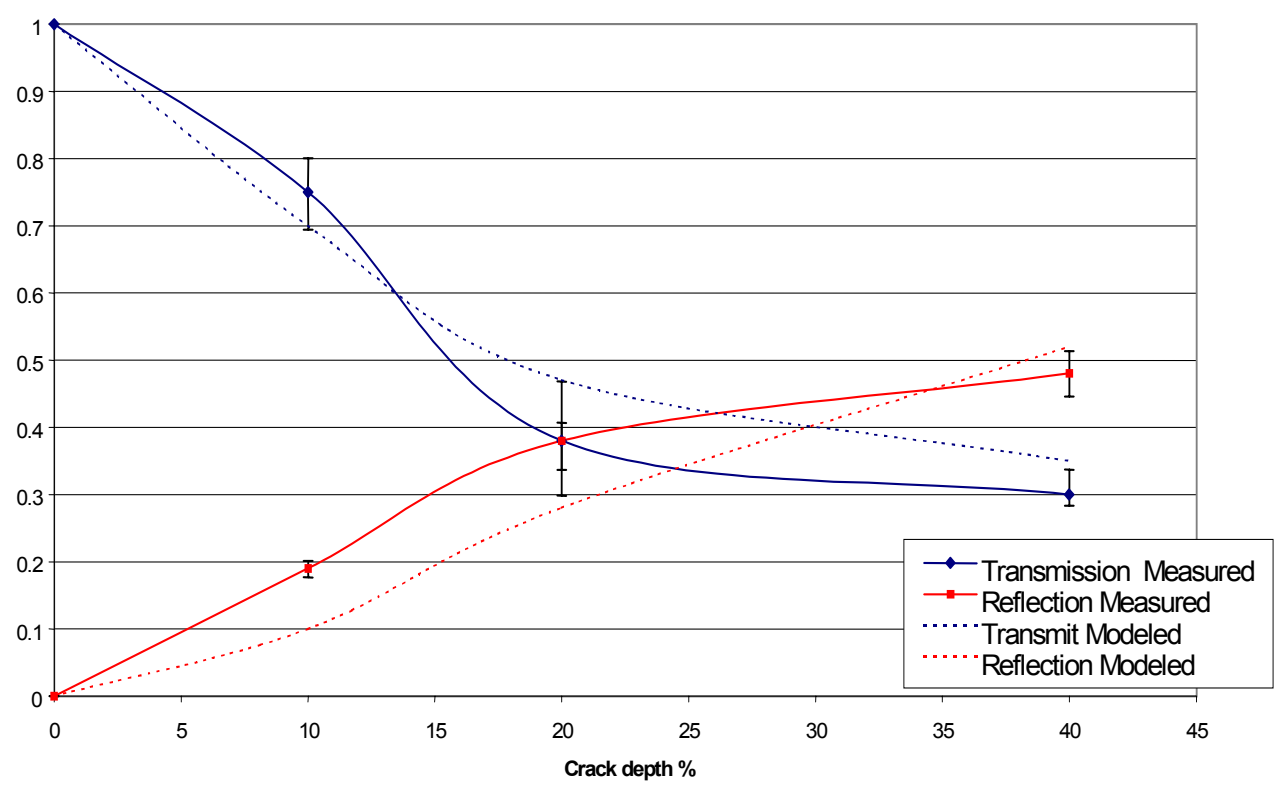

Figure 37 The measured and modelled reflection and transmission coefficients for interaction of the SV1 mode at 3.9 MHz-mm with a cracks of varying depth.

Transmission \& Reflection Coefficient Results For SH0 @260kHz

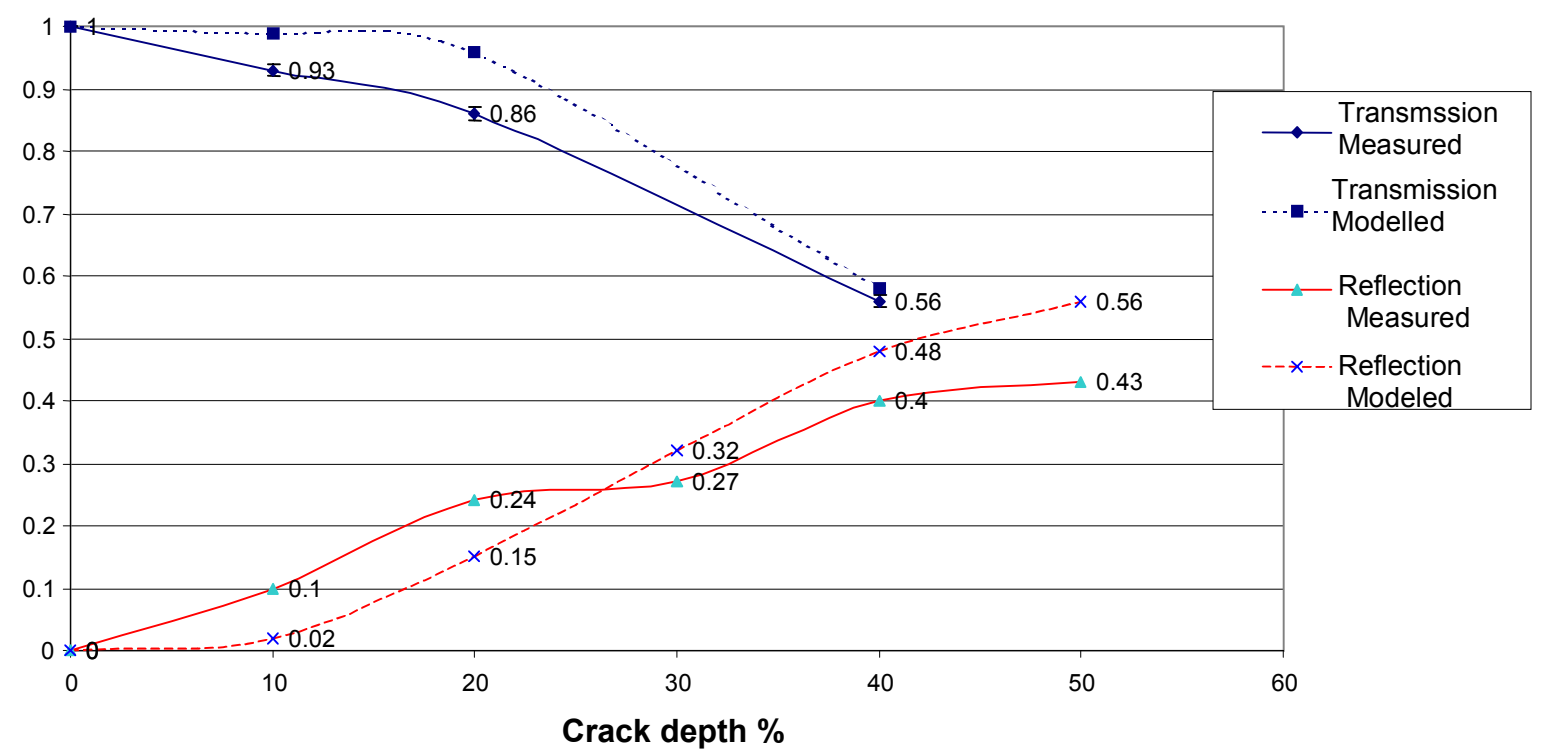

Figure 38 The measured and predicted reflection and transmission coefficients for interaction of the SHO mode at $2.1 \mathrm{MHz}-\mathrm{mm}$ with cracks of varying depth. 


\section{Detectability of SCC On a Sample Section}

All results were far from conclusive. For transmission measurements, as expected, a reduction in amplitude was observed and the maximum effect was near the deepest defect. For further details see the first annual report [13]. Any analysis of reflection data proved to be extremely difficult and speculative due to reflections from the edges, and the fact that the transmitter passed over a defect. Because of this and because we had seen enough SCC interaction for transmission measurements, work with SCC pipe sections was stopped in favor of work in full circumference pipes with a mouse.

\section{Lab Mouse Results}

\section{SV1}

As shown in Figures 37 and 38 mouse signal quality was good and there was definite crack interaction. Figure 36 shows an example trace from a spot without defects at the far end of the pipe. The trace clearly shows the following arrivals (in order): the firing breakthrough, the direct arrival (travelling counter clockwise 90 degrees), a small A1 mode arrival (travelling counter clockwise 90 degrees), the back-propagated arrival (travelling clockwise 270 degrees), a second direct arrival (travelling counter clockwise 450 degrees). The A1 arrival is a parasitic excited because its wavelength is similar to that of the desired SV1 mode. The A1 amplitude is about $1 / 20$ that of the direct amplitude, which indicates good modal purity. The group velocities of the arrivals, except A1, are estimated from arrival times and distances at about $4950 \mathrm{~m} / \mathrm{s}$, as expected for the SV1 arrival in .31" wall at $460 \mathrm{kHz}$.

Figure 38 shows a trace taken at the axial position of P2-3 that contains an additional reflected signal from a defect plus the arrivals noted for the previous trace. The defect reflection occurs where expected - about 109 us after the direct arrival.

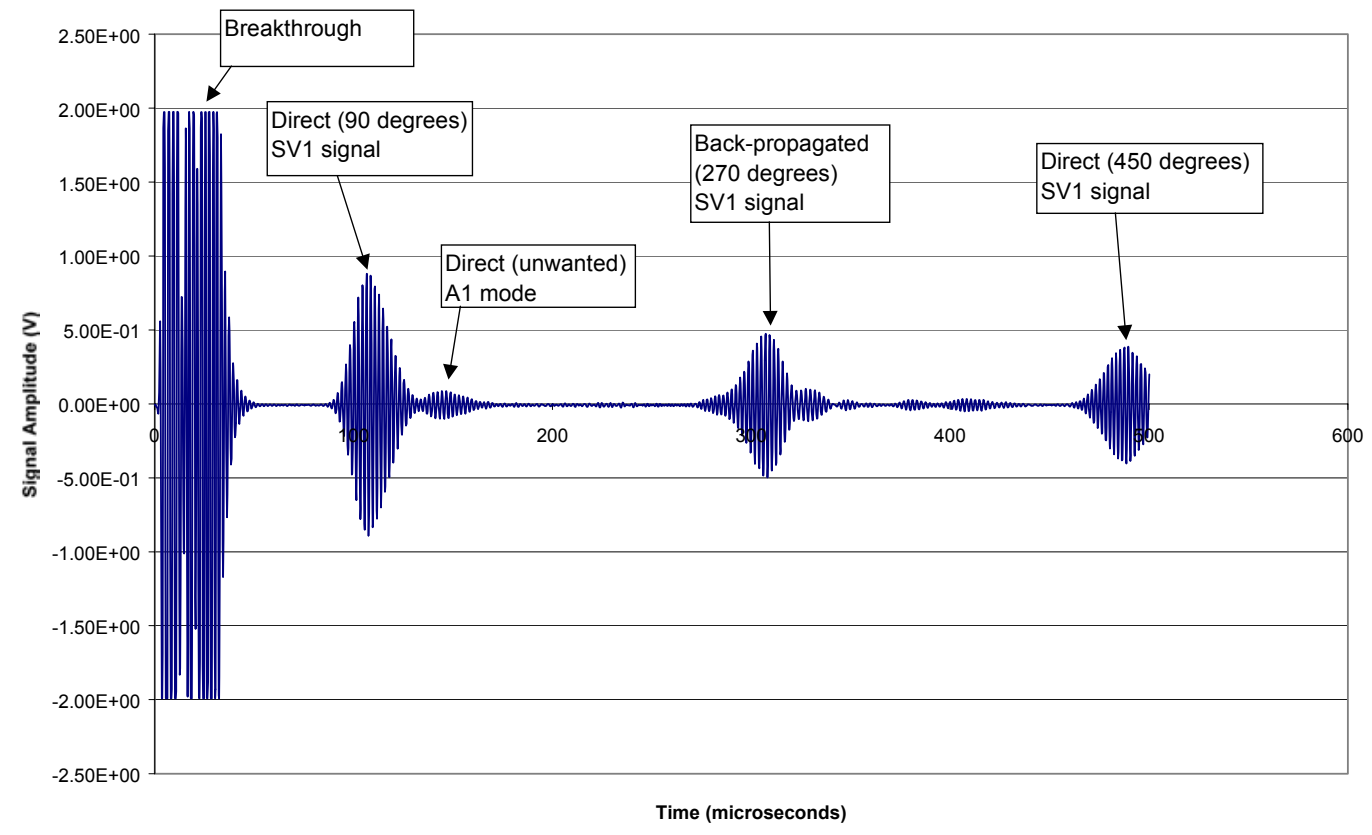

Figure 37 Example trace from the SV1 mouse carriage at a position $1180 \mathrm{~mm}$ along pipe (no defects present). 


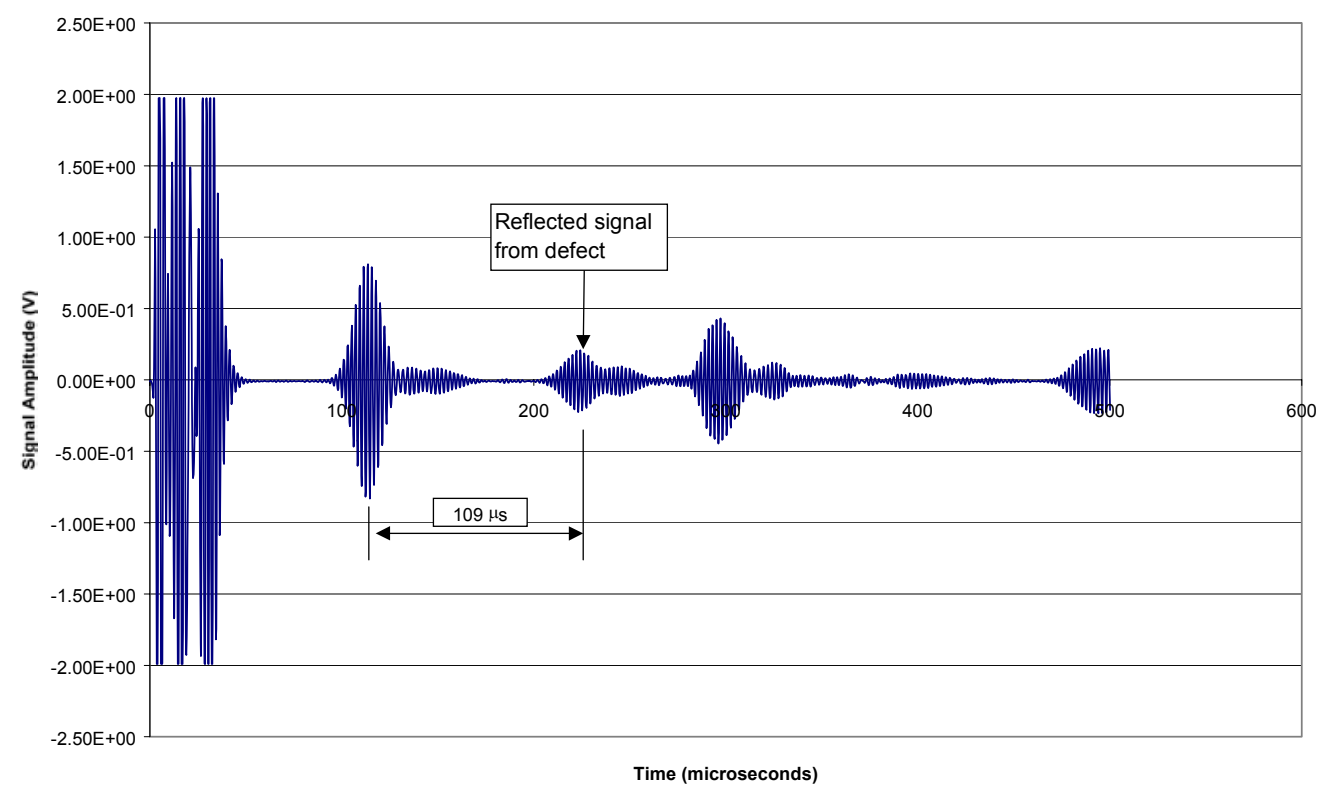

Figure 38 Example trace from the SV1 mouse carriage at a position $555 \mathrm{~mm}$ along pipe (defect present).

Figure 39 shows amplitudes for an SV1 $(473 \mathrm{kHz})$ reflection scan. In this figure the defect positions are marked by rectangles along the $x$ axis. The scale for the raw direct and reflection signal amplitudes (squares and triangles) is on the right y axis. These are somewhat arbitrary because they are determined by the gain and drive levels. One feature of the curves is that the direct amplitude varies greatly, by a factor of 10 over the axial length of the pipe. This is due to coupling variations caused by material effects at the transducers and transducer liftoff. Another feature is that there is a correlation between defects and reflected signal for the first five and the last defects.

The normalized reflection signal ${ }^{4}$ (blue diamonds) in Figure 39 circumvents direct amplitude variations and improves the correlation with the defects. The normalized is just the reflected signal divided by the direct amplitude. Its scale is on the left vertical axis. The normalized is basically a reflection coefficient, because it can vary from 0 to 1 . For the first two defects (300 $\mathrm{mm}$ and $450 \mathrm{~mm}$ ) it has the values of .26 and .15, which are expected for defects 15 to $25 \%$ of wall thickness. It measures less than .07 for the other defects, which may be closed or just not very deep.

The normalized reflections repeat well for larger defects, as shown by Figure 40 for repeat runs around the first three defects.

Figure 41 shows the direct arrival (blue diamonds) and other amplitudes for an SV1 transmission scan. It includes the backward arrival amplitude (green squares), which is labelled "around 1" because it goes backward nearly a full revolution. The around 1 and direct scales are on the right side y axis. The direct, which passes through the defects should be affected by them. The direct varies by a factor of 10 , but there is little correlation with the defects, because coupling variations are dominant.

\footnotetext{
${ }^{4}$ In the rest of the text we will refer to this as the normalized reflection.
} 


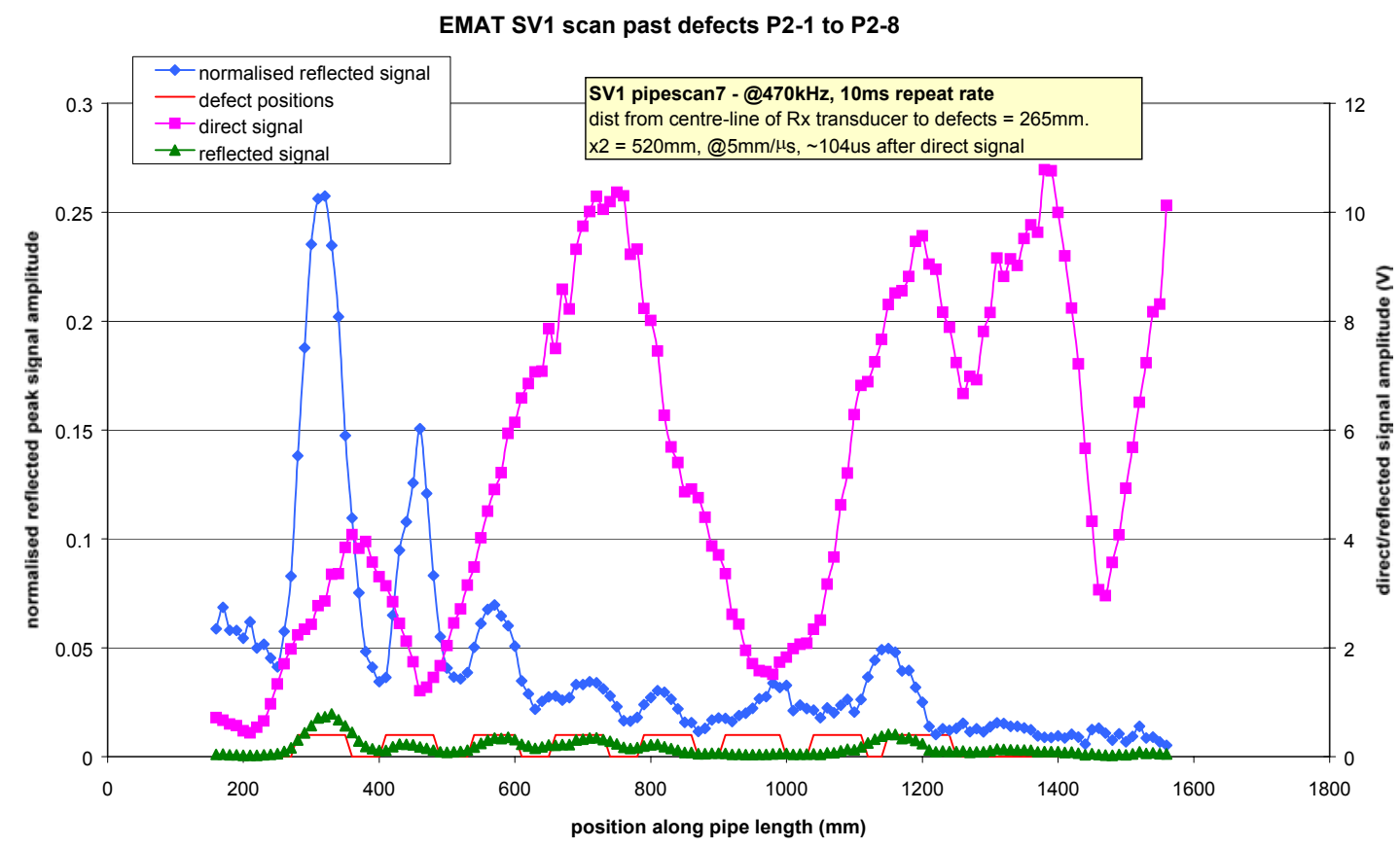

Figure 39 For the lab mouse the amplitude of the direct signal, the raw reflected signal ( 104 microseconds after the direct), and the normalised reflected plotted as a function of distance along pipe sample P2 for SV1 EMAT (defect locations as determined visually are shown).

EMAT SV1 reflection scans past defects P2-1, P2-2 and P2-3

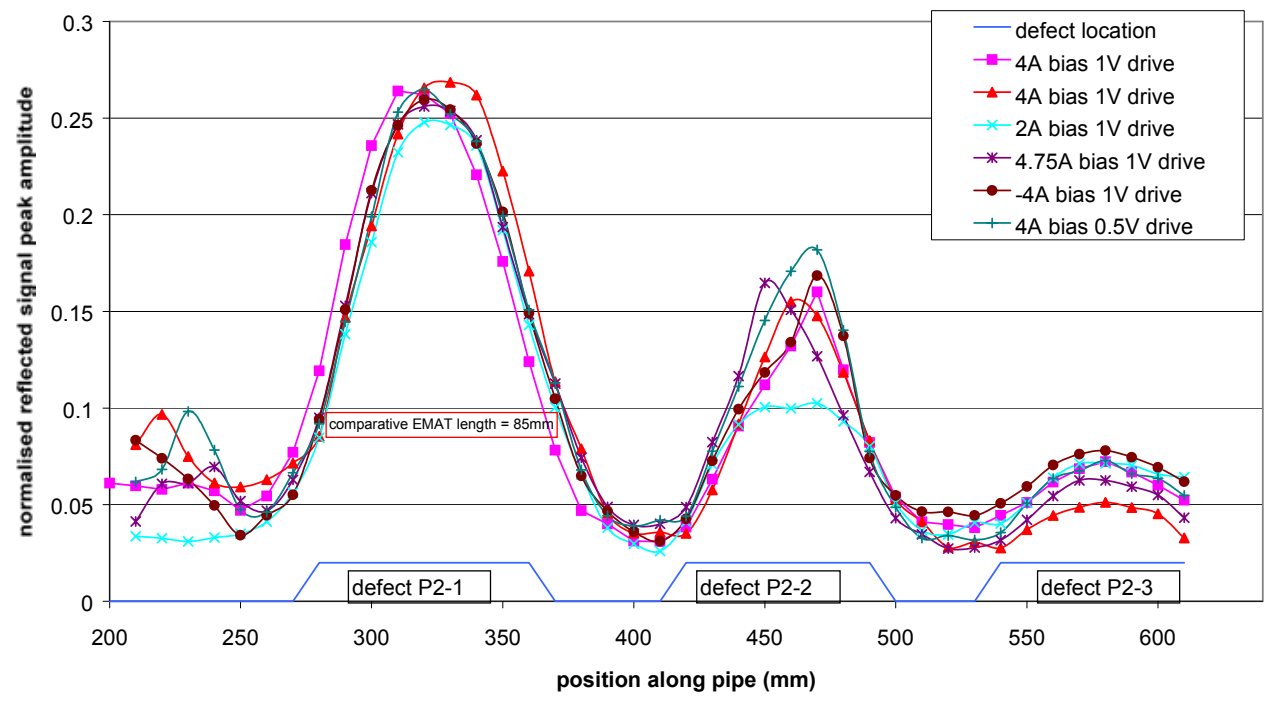

Figure 40 For the lab mouse the amplitudes of the normalised reflection (at 104 microseconds after the direct signal) plotted as a function of distance along pipe sample under varying bias field and drive voltage conditions (Refer to the legend for plot symbol and conditions.)

\section{Tuboscope Pipeline Services}




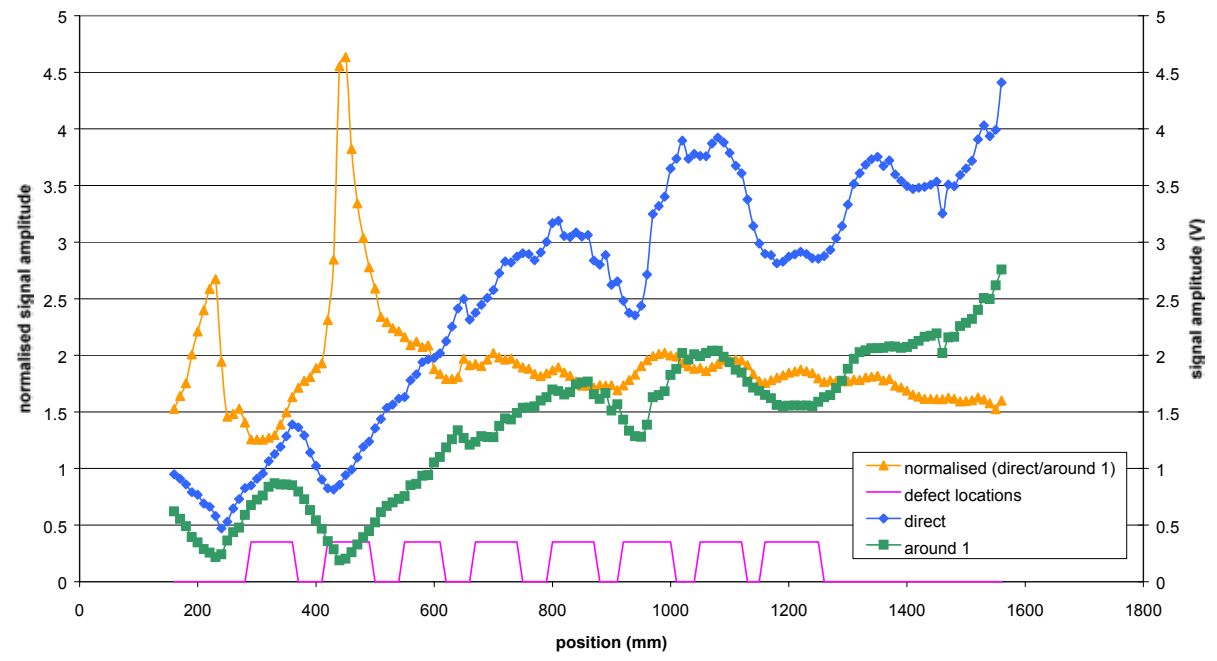

Figure 41 For the lab mouse the amplitude of the direct signal, back-propagated signal, and the normalised direct signal plotted as a function of distance along pipe sample P2 for SV1 EMAT in transmission set-up.

The "around 1", which goes backward around the defects should not be affected by them even though it may be subject to coupling variations. Thus, it could be used to normalize the direct to remove coupling effects. This is what is done for the normalized (gold triangles). The scale of the normalized is on the left axis of Figure 41. Unfortunately, the "around 1" is subject to losses (spreading and radiation), so that the direct/around1 ratio is greater than 1. More significantly, the normalized does not correlate well with the defects, and it peaks where it actually should have a minimum at defect P2-2

A correction can be done in which the normalized curve is divided by a constant such that a point where there is no expected loss is set to unity. Unfortunately, this did not help remove the peak near $400 \mathrm{~mm}$ (defect 2) or improve interpretation of the resulting curve.

Note that the previous techniques of display (with different scales on the left and right vertical axes) and normalization will be used in the remainder of this report.

SH0

As shown in Figures 42 (defect-less) and 43 (with a reflection from defect P2-1) SH0 mouse results showed good signal quality and crack interactions. The arrival content was similar to the SV1 traces in Figures 37 and 38, except for a few differences: First, the arrival times (and time between the direct and defect reflections) are different because the $\mathrm{SHO}$ mode group velocity speed is slower, estimated at about $3250 \mathrm{~m} / \mathrm{s}$ which is correct for the $\mathrm{SHO}$ mode. The round trip counter clockwise is absent because it is too late to be included. The mode of the small amplitude signal just after the $\mathrm{SHO}$ direct arrival is unknown. However, its amplitude is about $1 / 20$ that of the direct, which shows that the direct has good modal purity. 


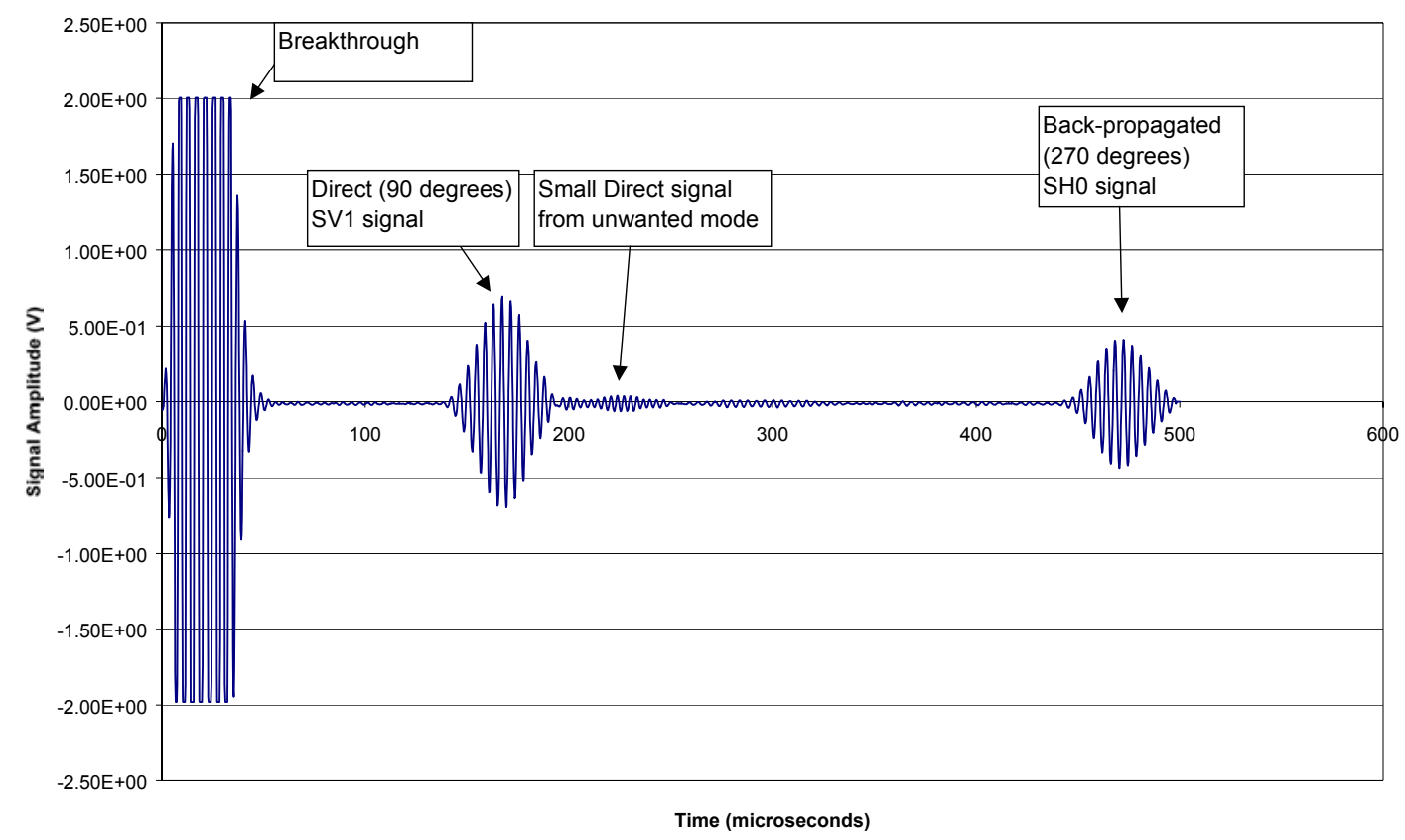

Figure 42 Example trace from the SHO mouse carriage at a position $1372 \mathrm{~mm}$ along the pipe (no defects present).

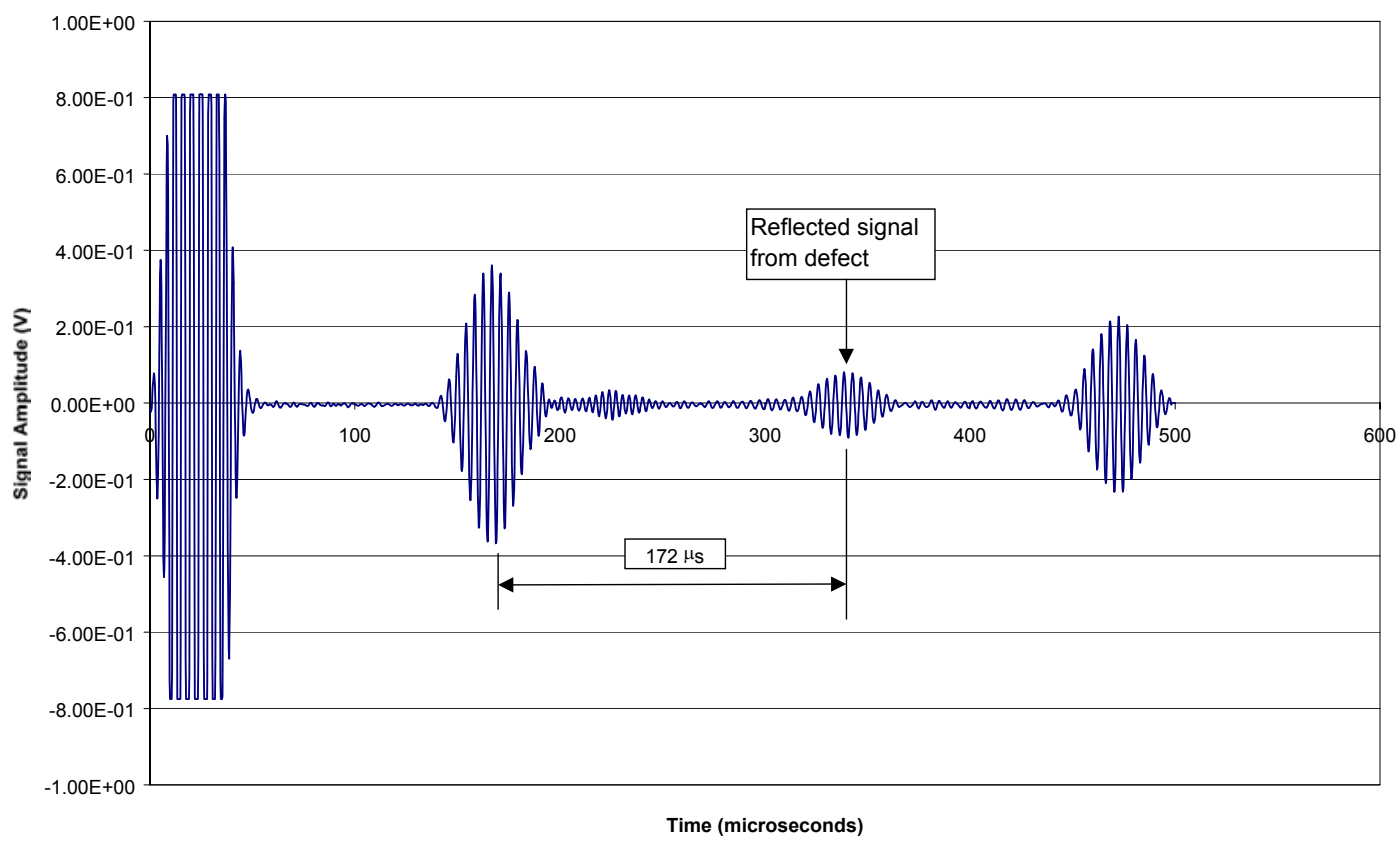

Figure 43 Example trace from the SHO mouse carriage at a position $322 \mathrm{~mm}$ along the pipe (defect present).

Figure 44 shows scan amplitudes for SHO (at $260 \mathrm{kHz}$ ). The curves are similar to those of Figure 39 in derivation. SHO direct amplitude (plotted diamonds) varies but over a smaller range (2 to 1 ) than the SV1. It was found that the variation was mostly due to standoff 
variations. The normalized (reflection, plotted x's) has a fair correlation with the defect locations. The level for this first defect (.24) is about what was expected for a defect about $20 \%$ to $25 \%$ of wall thickness.

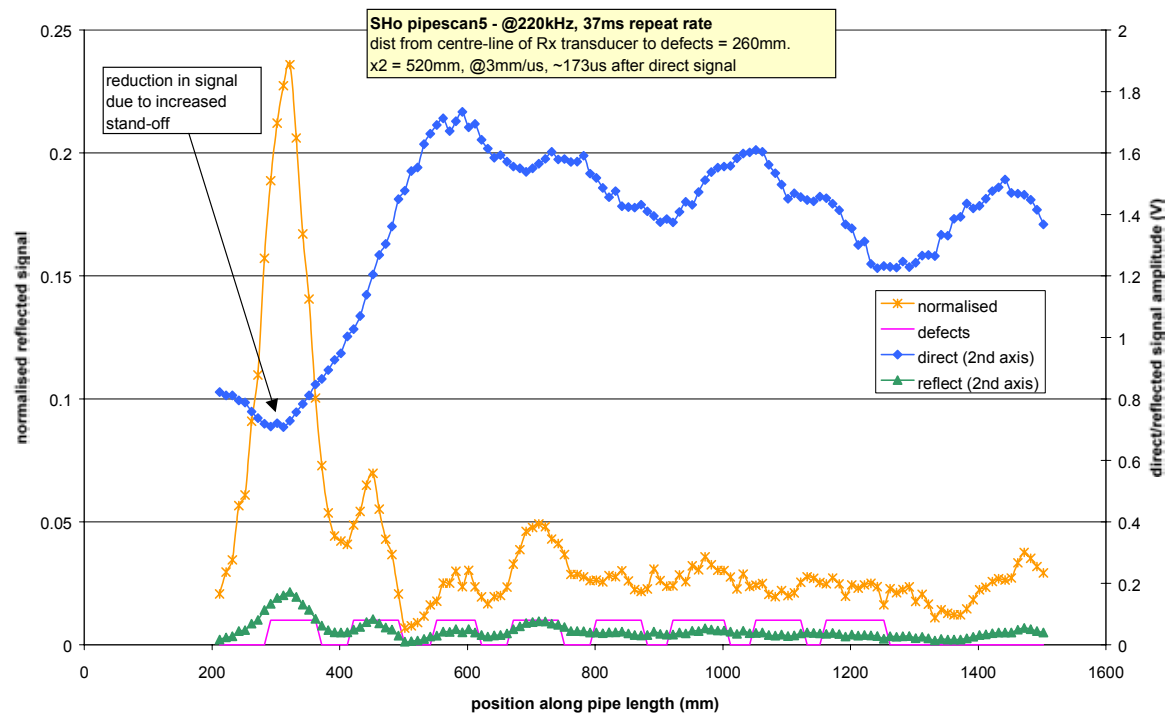

Figure 44 For the lab mouse: the amplitude of the direct signal, reflected signal at $\sim 172$ microseconds after the direct signal, and the normalised reflected plotted as a function of distance along pipe sample P2 for SHO EMAT set-up \#1 (defect locations as determined visually are shown).

Figure 45 shows $\mathrm{SH} 0$ transmission measurements. Because the variation of direct amplitude was not too large in Figure 45, it was hoped that the transmission scan would be useful. Plotted curves (direct(2nd axis), around(2nd axis), and normalized) are similar to those of Figure 41 for

pipescan SH0 7, defects P2-1 to P2-8, transmission response

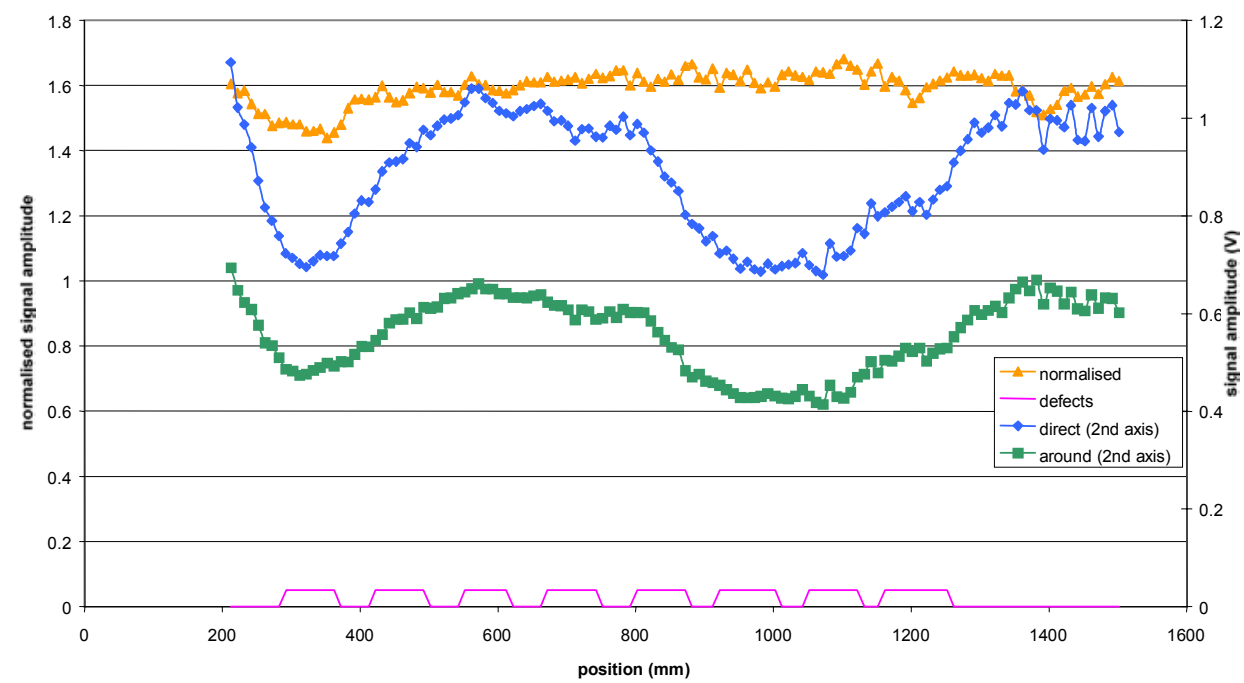

Figure 45 For the lab mouse: the amplitude of the direct signal, back-propagated signal, and the normalised direct signal plotted as a function of distance along pipe sample P2 for SHO EMAT in transmission set-up (defect locations are shown). 
SV1 and derived and displayed similarly. The normalized (plotted gold triangles) has a weak decrease near the first defect. Like the SV1 normalized transmission the value is more than unity. The normalized curve can be corrected, so that it is more like a transmission coefficient by dividing it by the average value at a normalization point (around $1500 \mathrm{~mm}$ ) where there are no defects. This was done and is shown in Figure 46. (Note dual axis scales.) At the normalization point the value of the corrected-normalized (gold triangles) is unity, thus denoting no defects. At the first defect (at $\sim 300 \mathrm{~mm}$ axially) the weak depression in the normalized becomes .88 in the corrected-normalized curve. If this is taken as the transmission coefficient, the crack depth would be about $16 \%$, which is low. Because of this and the problems of normalization to remove coupling variations, we believe that transmission measurements will not be useful in grading SCC.

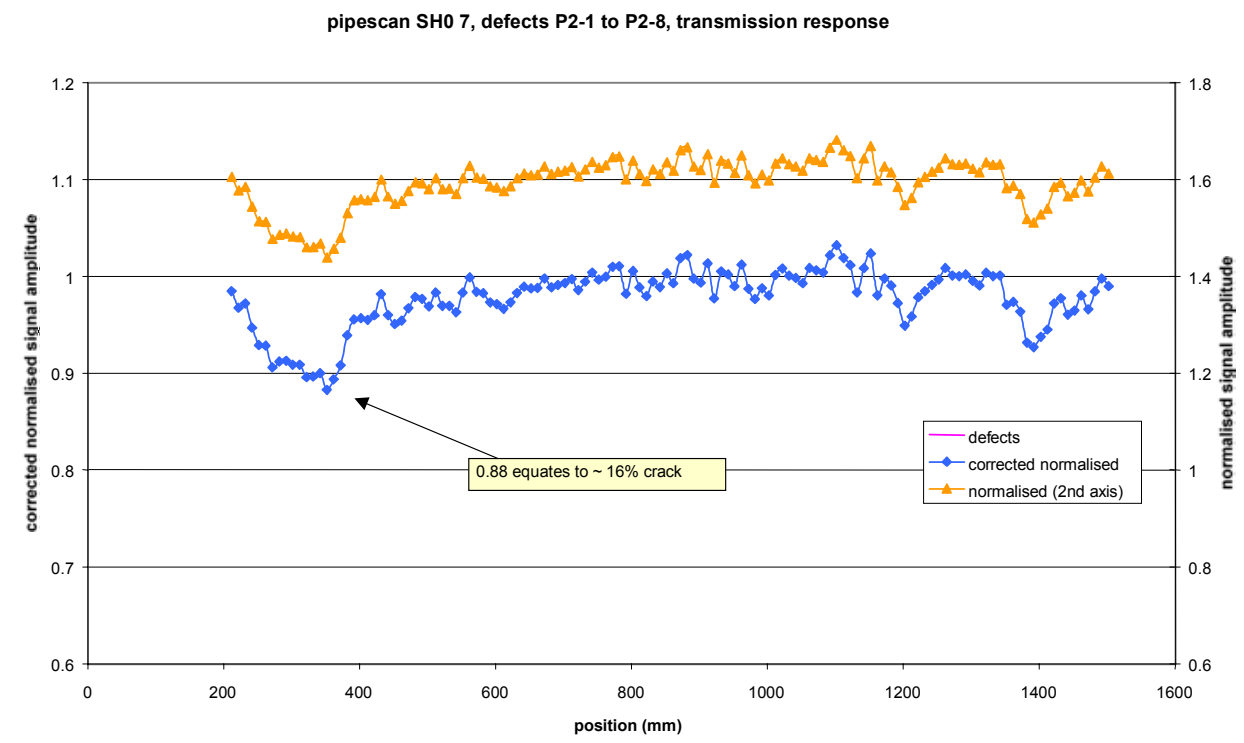

Figure 46 For the lab mouse: the amplitude of the normalised and corrected-normalised direct signal plotted as a function of distance along pipe sample P2 for SHO EMAT in transmission setup. Note the corrected-normalized scale is the left and the normalized is the right vertical axis.

\section{$\underline{\text { Mule Developments }}$}

Prototype Circuits

The prototype transmitter driver system (Figure 21) was able to drive the 2 layer ( $\sim 1$ ohm) EMAT to + - $75 \mathrm{~A}$ for a 5 cycle drive at $470 \mathrm{kHz}$ and at a pulse rate of $500 \mathrm{~Hz}$. This system ran off a $20 \mathrm{~V}$ battery supply line, drawing $1 \mathrm{~A}$, for a power input of $20 \mathrm{~W}$. The high voltage supplies stepped up the $20 \mathrm{~V}$ to + and $-100 \mathrm{~V}$ for the power pulser. Energy storage in the supply system was able sustain the firing up to 10 cycles at a $500 \mathrm{~Hz}$ repetition rate without any degradation in the transmitted pulse. The performance and size of this system was excellent compared to the mouse. The peak current level was twice that of the mouse and was obtained in much less volume.

The receiver preamp and filter had a gain of $98 \mathrm{db}$ and bandwidth of $250 \mathrm{kHz}$. The noise spectral density referred to the input was $0.3 \mathrm{nV} / \mathrm{sqrt}(\mathrm{Hz})$ at the center frequency. At the output that means the rms noise was only $25 \mathrm{mV}$ - a relatively low level for a maximum signal range 
of $9 \mathrm{~V}$ peak to peak. For the 30" pipe setup for $\mathrm{SHO}$ with permanent magnet EMATs (Figure 23) and the $75 \mathrm{~A}$ firing circuit (Figure 21) the peak signal level was $3 \mathrm{~V}$. This corresponds to a signal to noise ratio of 80 , which is good considering the permanent magnetic bias levels were less than with the mouse.

The transmitter control had a special provision in the timing to randomly jitter the firing rate by a small percentage. This was used to reduce the noise from multiple echoes from previous firings that can persist because of very low attenuation and that can interfere like noise ${ }^{5}$. They are often called, "rep rate noise". Figure 47 shows an example of the previous noise-like effects and subsequent reduction by at least 3 to 1 with modest averaging in conjunction with the jitter circuit. All the logic was encompassed in a single gate array along with other transmitter logic.

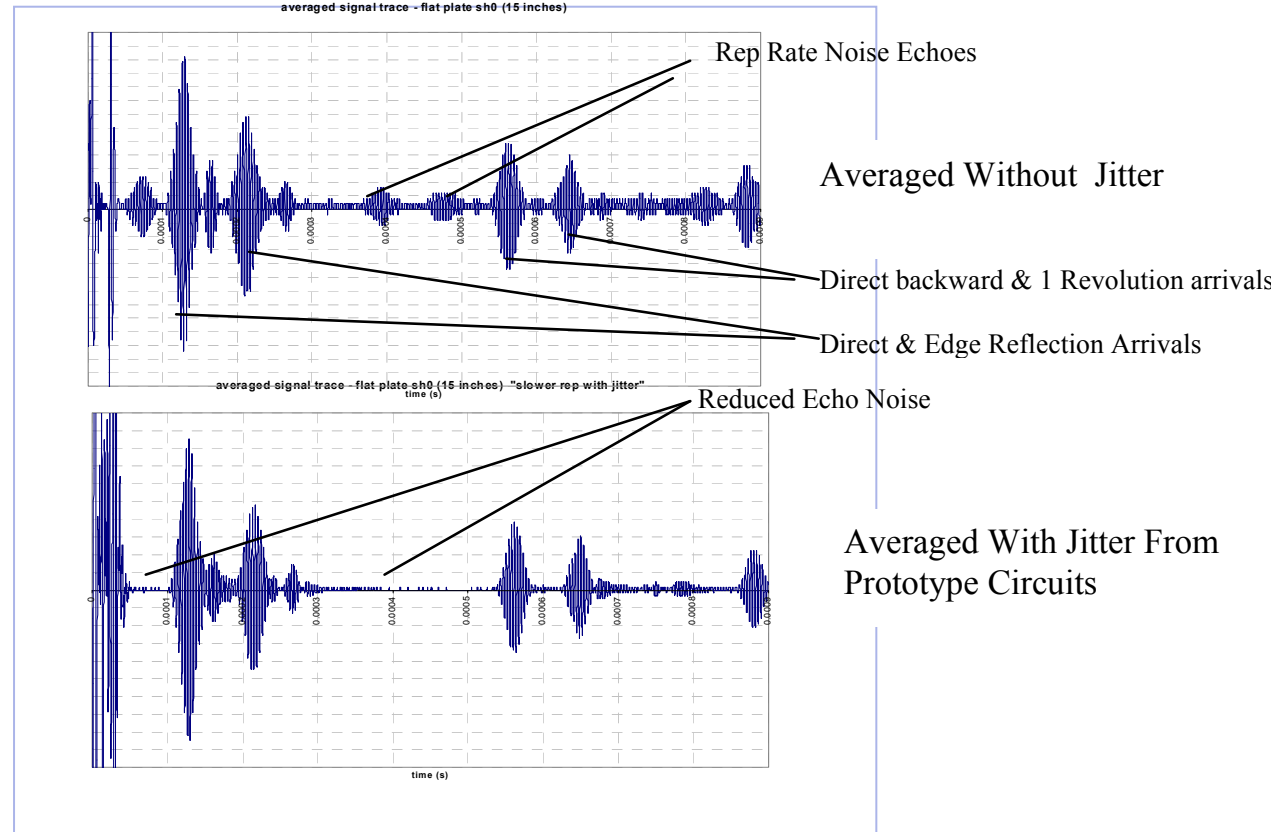

Figure 47 Mule prototype flat plate arrivals in averaged signals with and without firing rate jitter, showing rep rate noise or echo amplitudes reduced by jitter.

Acquisition Specifications and Configuration

Table 3 shows practical mule data acquisition specifications based on lab mouse and mule experiences with a digital scope. The sample rate provides enough data points to accurately determine the peak magnitude digitally and enough data for performing any further detailed analysis (e.g. FFT or digital filtering). The data interval provides enough time for the $\mathrm{SHO}$ mode (at the chosen frequencies) to travel fully around the pipe and for a receiver to look ahead half way around the pipe. To inspect the whole circumference would require at least two receivers. If more receivers and transmitters are used, the digitization interval and the number of samples can be reduced.

\footnotetext{
${ }^{5}$ If the firing rate is fixed, echo energy is stationary and of constant amplitude in the received signal, and so even if the received signal is averaged over many firings, the desired signal to echo amplitude ratio stays the same. However, if the firing rate is jittered randomly, as done by our prototype, the echoes have random phase and amplitude, and decrease with averaging like random noise does. Thus, with the jitter circuit the signal to these echoes (rep rate noise) increases with averaging.
} 
Because of the lack of sensitivity of transmission measurements, the mule was configured for reflection measurements only. The configuration was for pitch-multiple catch by means of a pair of receivers with a transmitter in between them, as in Figure 20, with 45 degrees between each transducer.

Table 3 Data Acquisition Specifications For An EMAT System

\begin{tabular}{|l|l|l|}
\hline Parameter & Value & Remarks \\
\hline Digitizer Voltage Resolution & 8 to 12 bits & At least \\
\hline Digitizer Sample Rate & $2.5 \mathrm{MHz}$ & \\
\hline Digitized Interval (time) & $750 \mathrm{us}$ & Per receiver (30" Pipe) \\
\hline \multicolumn{1}{|c|}{ (samples) } & 1875 samples & For 360 degree coverage \\
\hline Transmitter Drive & 4 or 5 cycle tone burst & \\
\hline Transmitter Drive sample rate & $5 \mathrm{MHz}$ & \\
\hline Firing Rate & 10 to $500 \mathrm{~Hz}$ & Per transmitter head \\
\hline Transmitter Drive Frequencies & $260 \mathrm{kHz}$, to $460 \mathrm{kHz}$ & \\
\hline Transmitter Instant. Power & $140 \mathrm{Watts}$ & Minimum \\
\hline Transmitter Average Power & 20 Watts & \\
\hline
\end{tabular}

$\underline{\text { Additional Measurements With Mule Circuits }}$

Dispersion

In agreement with theory, SHO measurements of group and phase velocity (Figure $48 \mathrm{~A}$ ) in plates (from 0.30 " to $0.50 "$ thick) are nearly constant versus thickness and show the nondispersive nature of this mode versus plate thickness. This non-dispersive nature of $\mathrm{SHO}$ is very advantageous because it means that the envelope of the probing wave will not spread with distance and that circumferential distance is linearly proportional to travel time by a constant $(3250 \mathrm{~m} / \mathrm{s})$, independent of thickness or frequency.

For SV1 (at $460 \mathrm{kHz}$ ) the group velocity measurements (Figure $48 \mathrm{~B}$ ) agree decently with theory and show a large dispersion of $40 \%$ from a wall thickness of 0.30 to 0.50 inches. This agrees fairly well with theory for SV1 at that frequency. The phase velocity measurements follow the trend of the theory, also indicating dispersion, but are generally smaller by $1000 \mathrm{~m} / \mathrm{s}^{6}$.

Due to SV1 dispersion, a small change in thickness will affect direct and reflection envelopes, decreasing them more than spreading loss and will complicate the time to circumferential distance transformation. Because both effects complicate the analysis of SV1 reflections, further investigation of SV1 in the mule was dropped.

\footnotetext{
${ }^{6}$ The reason for this bias is under investigation but may be due to the fact that dispersion makes it harder to measure phase velocity.
}

Tuboscope Pipeline Services

47 of 59 


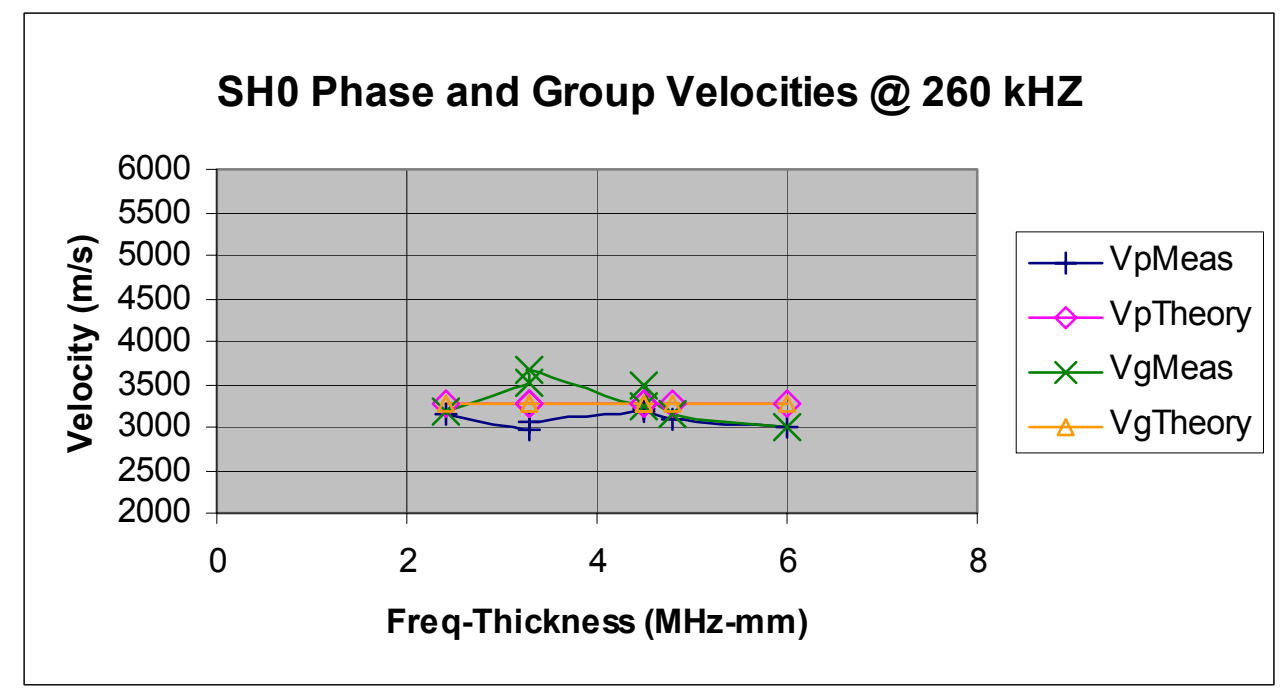

Figure 48 A SHO Phase and group velocities for various plate thicknesses. See Introduction for theoretic velocities.

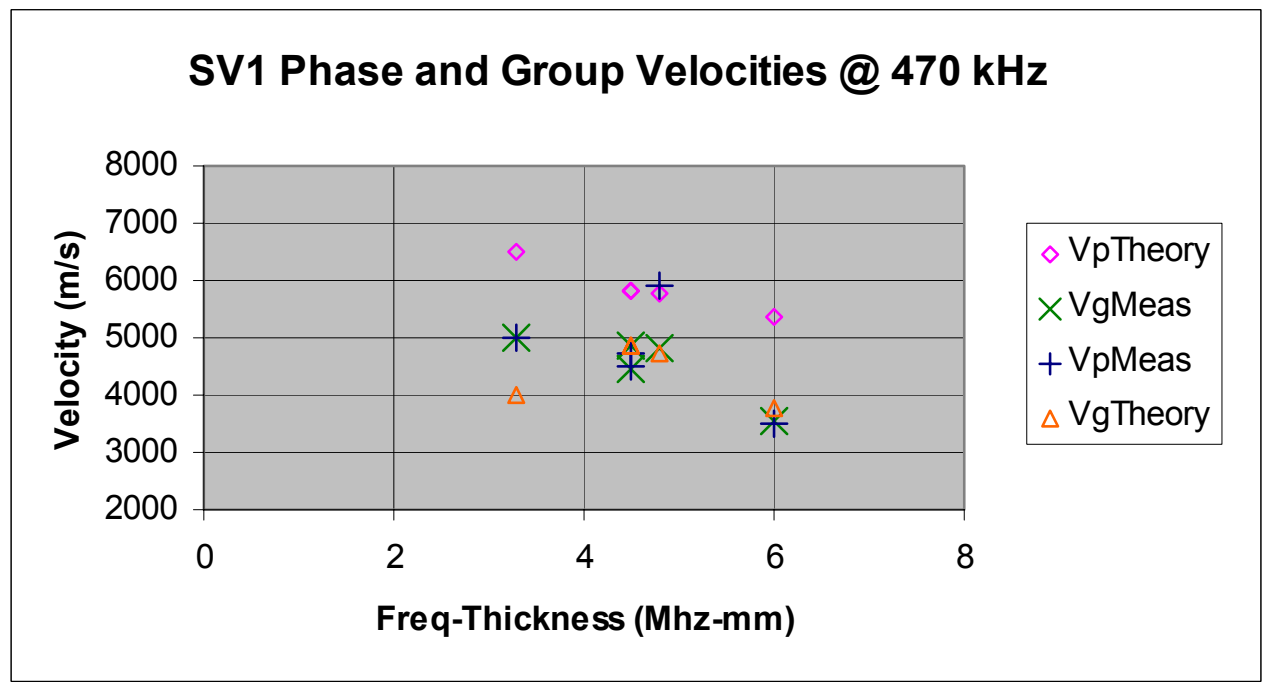

Figure 48 B SV1 Phase and group velocities for various plate thicknesses. Note that $0.2^{\prime \prime}$ thickness (2.4 MHz-mm) is omitted because SV1 is cutoff at $470 \mathrm{kHz}$. See Introduction for theoretic velocities.

Attenuation Due To Exterior Coating

As described in the previous section, waveform arrival measurements were taken in three conditions. The peak data, separation distances, and calculations are shown in Table 4 and summarized in Table 5. Attenuation measurements from transmitter to receiver separations of 16 and 22 inches, corresponding to travel distances of 61.3 and 49.3 inches, were averaged to obtain the summary values. The amount of attenuation of one or two layers is tolerable for one revolution (93 inches) around the pipe. Thus, we believe a transmitter-receiver pair could inspect half or more of the pipe circumference. 
Table 4 Raw Data and Attenuation Calculations

\begin{tabular}{|c|c|c|c|c|c|c|c|c|}
\hline EMAT Signal Atte & nuation & Raw D & ta and Calulat & on WS & & & & \\
\hline \begin{tabular}{l|l} 
for $263 \mathrm{kHz}$ \\
\end{tabular} & $\mathrm{SHO} \mathrm{Mod}$ & e in 30 & .312 WT Pip & & & & & \\
\hline & Raw Da & & & & & & & \\
\hline & Vpp Sig & nal $(V)$ & Trav Dist (in.) & Amp Fraction & Atten $^{*} \mathrm{~dB} / \mathrm{in}$ & Tot Atten dB & Path Dis & t (in) \\
\hline Arrival & Direct & Back & Back-Direct & Dirct to Back & Dir to Back & DirBack & Direct & Back \\
\hline 16in UnCoat & 7.26 & 4.97 & 61.3 & 0.685 & -0.054 & -3.29 & 16 & 77.3 \\
\hline 22in UnCoat & 6.91 & 5.15 & 49.3 & 0.745 & -0.052 & -2.55 & 22 & 71.3 \\
\hline Average & & & & & -0.053 & & & \\
\hline & & & & & & & & \\
\hline 16in 1Coat & 6.83 & 2.56 & 61.3 & 0.375 & -0.139 & -8.52 & 16 & 77.3 \\
\hline 22in 1Coat & 6.92 & 3.39 & 49.3 & 0.490 & -0.126 & -6.20 & 22 & 71.3 \\
\hline Average & & & & & -0.132 & & & \\
\hline & & & & & & & & \\
\hline 16in 2Coat & 6.88 & 2.05 & 61.3 & 0.298 & -0.172 & -10.52 & 16 & 77.3 \\
\hline 22in 2Coat & 6.62 & 2.54 & 49.3 & 0.384 & -0.169 & -8.32 & 22 & 71.3 \\
\hline Average & & & & & -0.170 & & & \\
\hline & * Atter & $\mathrm{n}$ in $\mathrm{dB} /$ & $=$ Tot Atten/ & Trav Dist) & & & & \\
\hline
\end{tabular}

Table 5 Attenuation Summary

\begin{tabular}{|l|l|l|r|}
\hline Attenuation Summary ( SH0 263 kHz) & \\
\hline & \multicolumn{2}{|l|}{ Due to Coating Alone } \\
\hline Situation & Attenuation & Attention for 93" \\
\hline & db/in & db \\
\hline 1 Layer Coating & -0.13 & -12 \\
\hline 2 Layer Coating & -0.17 & -16 \\
\hline No Coating (Baseline loss) & -0.05 & -5 \\
\hline & & & \\
\hline & & & \\
\hline
\end{tabular}

\section{Mule Processing}

Processing was developed for the mouse and mule data reduction to produce the normalized reflection signals. This processing could also do data reduction and channelization of the data for display and feature extraction by means of standard Tuboscope LinaView software. Typical displays are $\mathrm{A}$ and $\mathrm{C}$ scans. In this processing, which is done to the digitized samples, the raw waveform is first bandpass filtered in a narrow band in order to reduce noise. Then its envelope is extracted, low pass filtered and resampled at a lower rate, and normalized by dividing by the direct arrival amplitude. This gives time samples of the normalized reflection, a reflection coefficient versus time or distance. The time samples are turned into circumferential distance by means of the group velocity ${ }^{7}$ as follows:

\section{Circumferential distance $=($ Group Velocity $){ }^{*}($ Sample Time $) / 2$}

In the previous relation, the first sample and the zero time reference correspond to the start of transmitter firing. Note that direct forward or backward arrivals travel half as far as reflections, so distance is misrepresented for direct or backward arrivals. Circumferential distance for reflections can be turned into orientation (in degrees) by dividing by radius and then multiplying the result by $180 /$ pi.

\footnotetext{
${ }^{7}$ For SH0 the group velocity is $3250 \mathrm{~m} / \mathrm{s}$.
}

Tuboscope Pipeline Services

49 of 59 
The whole processing can be done by a FPGA and/or DSP processor ahead of the data storage. The stored data can later be processed similarly to MFL data. Note that only data after the direct arrival and before the backward arrival need be stored. This can save some storage and memory access bandwidth.

Figures 49 through 52 illustrate the processing and display for the 30 " full pipe SHO data from mule prototypes. These data, 23 waveforms, were taken as an axial scan of the 30" pipe. Refer to Figure 24 for the geometry and Figure 23 for setup. For this data the interval includes the backward arrival and the forward arrival from a full revolution of the tool. These are all marked on Figures 50 and 51.

Note that for this setup the noise level of the normalized reflection is about 0.1 . This level is a cut-off for contouring in Figures 51 and 52. Below this level no contours are shown because there would be so many they would just appear as noise.

In Figure 52 the data is rotated, so it appears like a conventional pipeline $\mathrm{C}$ scan. The vertical axis is also shifted, so that 0 coincides with the receiver location. Because of this the direct arrival, shown by red is at 0 circumferential distance.

Figure 53 shows the A scan corresponding to Figure 52. For this figure the data was reduced by setting the circumferential resolution at 0.5 " (i.e. taking samples corresponding to $0.25 "$ circumferentially). This means taking only one of every forty-eight samples (decimation by 48) of the normalized reflection. Also, the samples before and during the direct arrival and after the backward direct arrival were dropped. This meant a reduction from 7300 samples (bytes) to 125 per trace. This is a reduction by a factor of 58 . We can drop these samples for the following reasons. The normalized direct arrival will always be 1 , so it is not useful. The data after the backward direct arrival could be backwards travelling energy not reflections, so it is ambiguous and nearly useless.

It should be noted that the waveform samples kept and also the portion of arrivals displayed in Figures 52 and 53 , cover only $38 \%$ of the circumference. A full coverage system would require 3 to four times as much data. For such, data per axial foot would increase but not data reduction. Also, this would require a similar increase in transmitter and receiver pairs, which should be uniformly spaced around the circumference. 
Full Pipe 1 SHO (263 kHz) Normd Env Ampl Scan 1 of M-M SCC , CCW direction

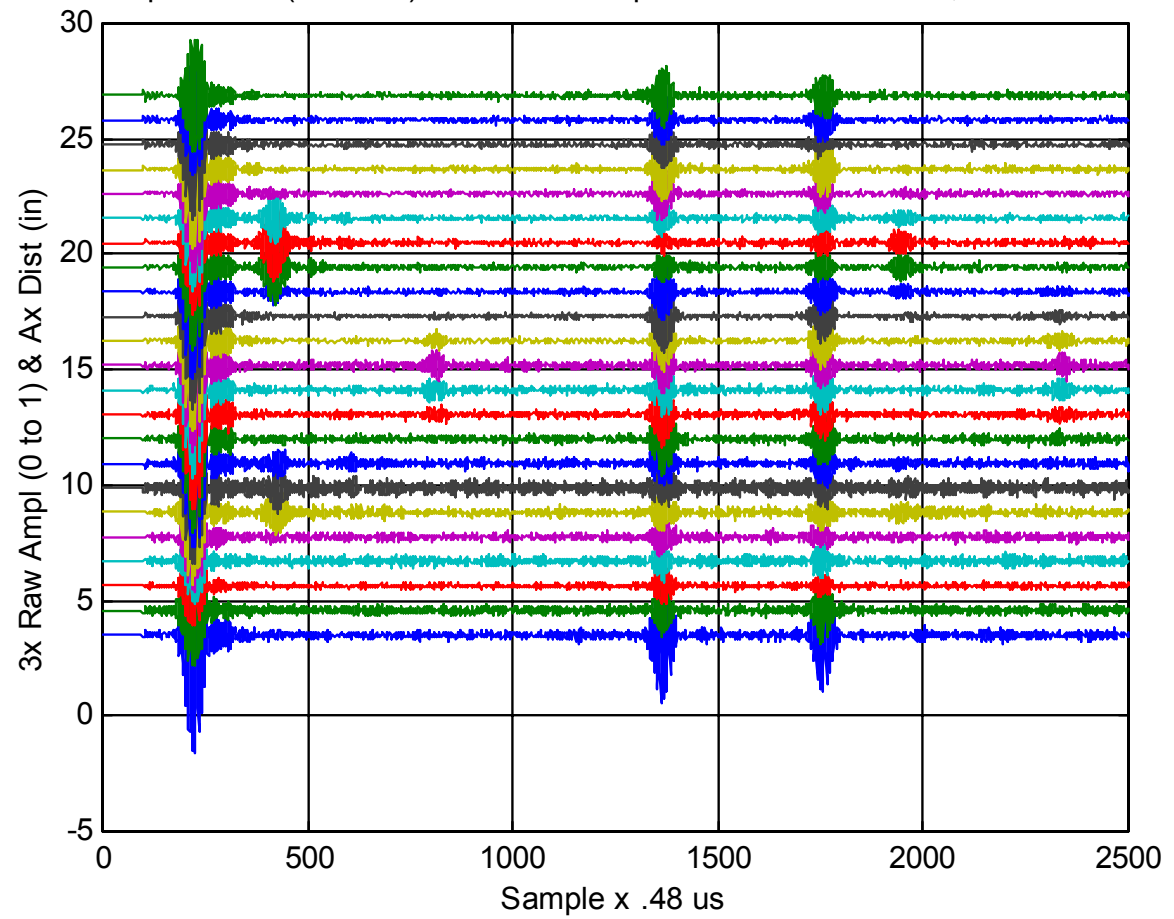

Figure 49 Raw data SCC scan reflection measurement waveforms taken with mule prototypes

Full Pipe 1 SH0 (263 kHz) Normd Env Ampl Scan 1 of M-M SCC , CCW direction

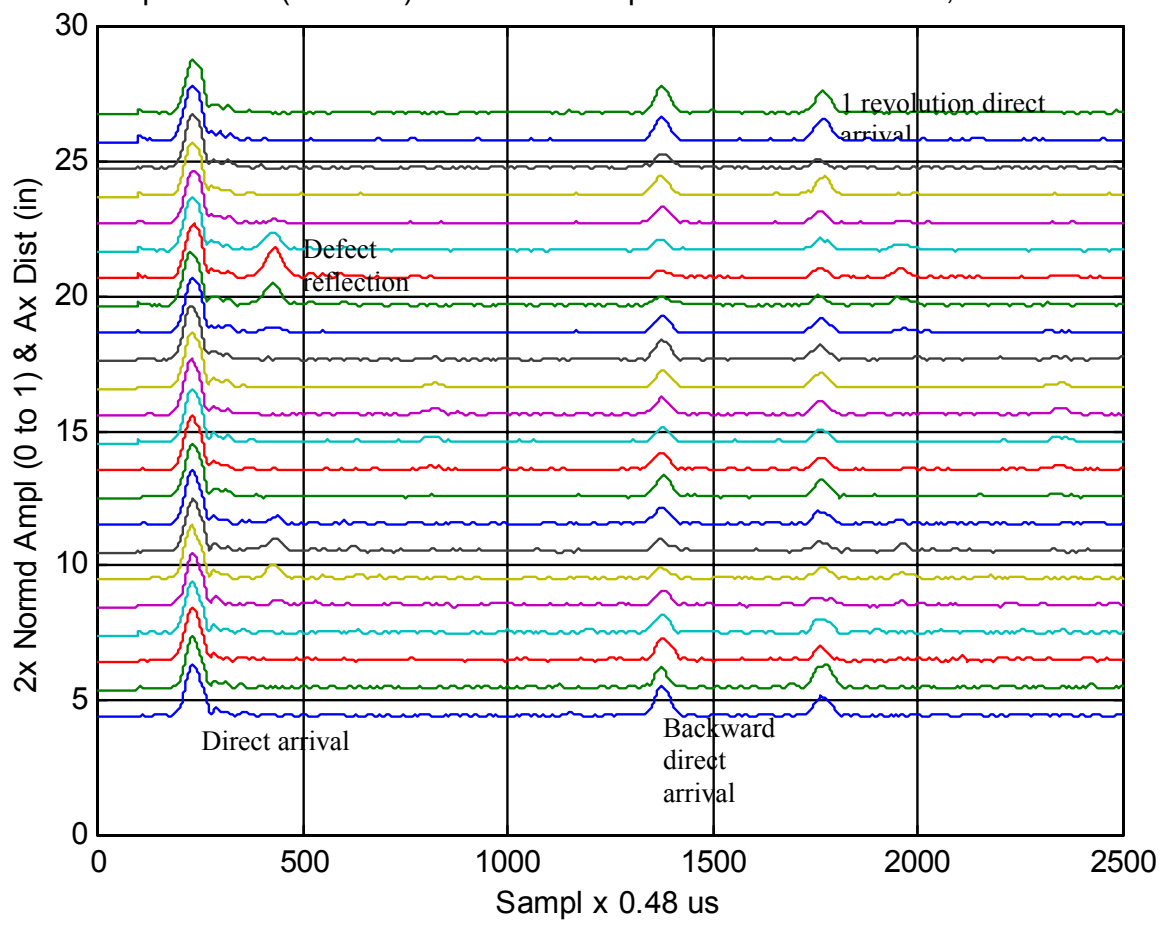

Figure 50 Envelope data of the SCC pipe scan (reflection) measurement with time axis, corresponding to Figure 49 
Full Pipe 1 SH0 (263 kHz) Normd Env Ampl Scan 1 of M-M SCC , CCW direction

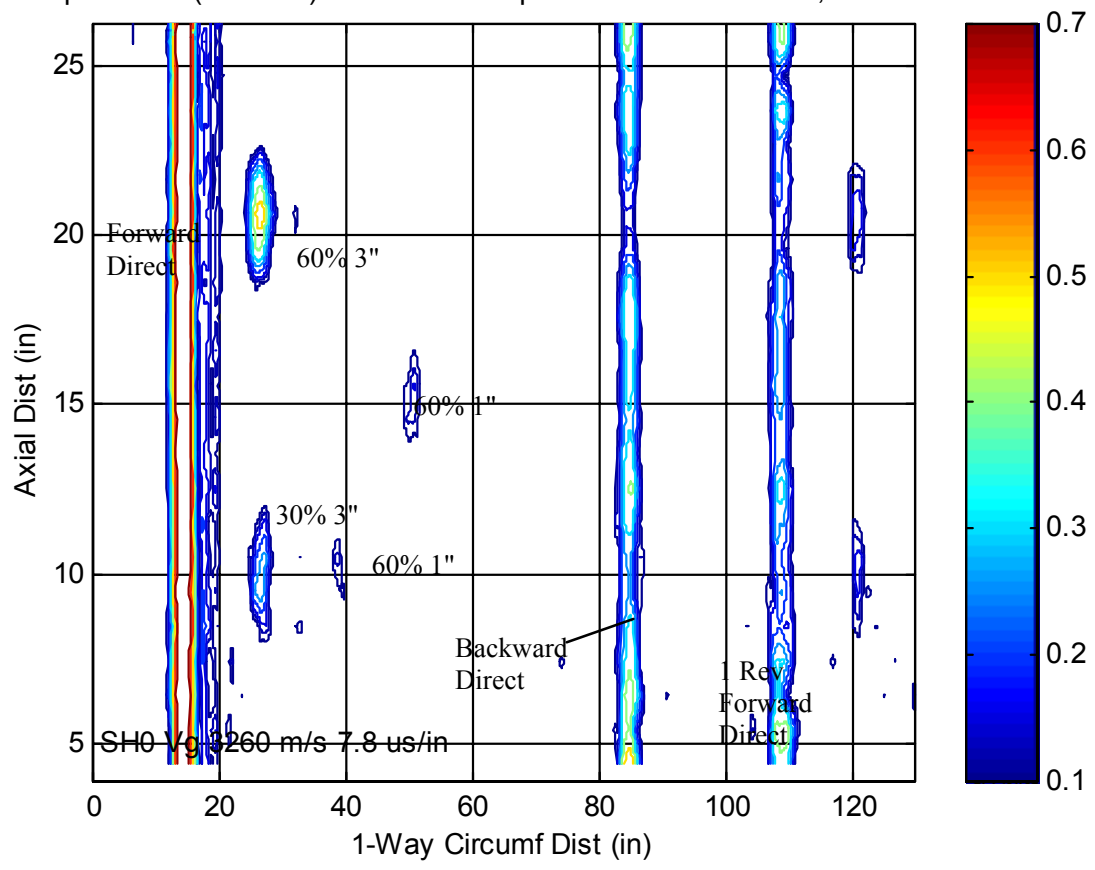

Figure 51 C Scan of envelope data corresponding to Figure 50. For the horizontal axis, time was converted to circumferential distance (for direct arrivals) from the transmitter.

Full Pipe 1 SH0 (263 kHz) Normd Env Ampl Scan 1 of M-M SCC , CCW direction

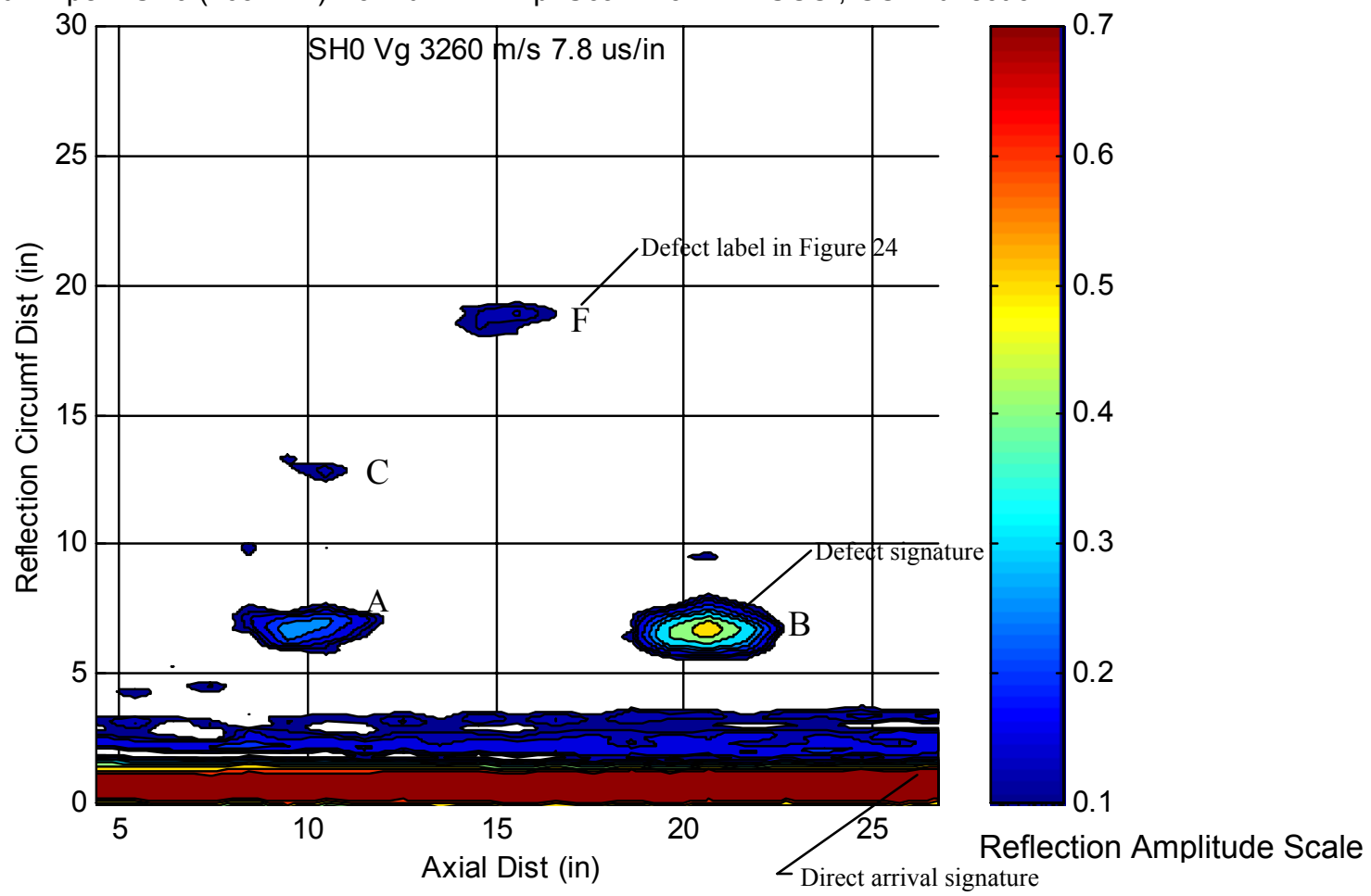

Figure 52 Rotated and enlarged C scan with axial and circumferential distance axes (time converted to circumferential distance for reflections), corresponding to Figure 51 


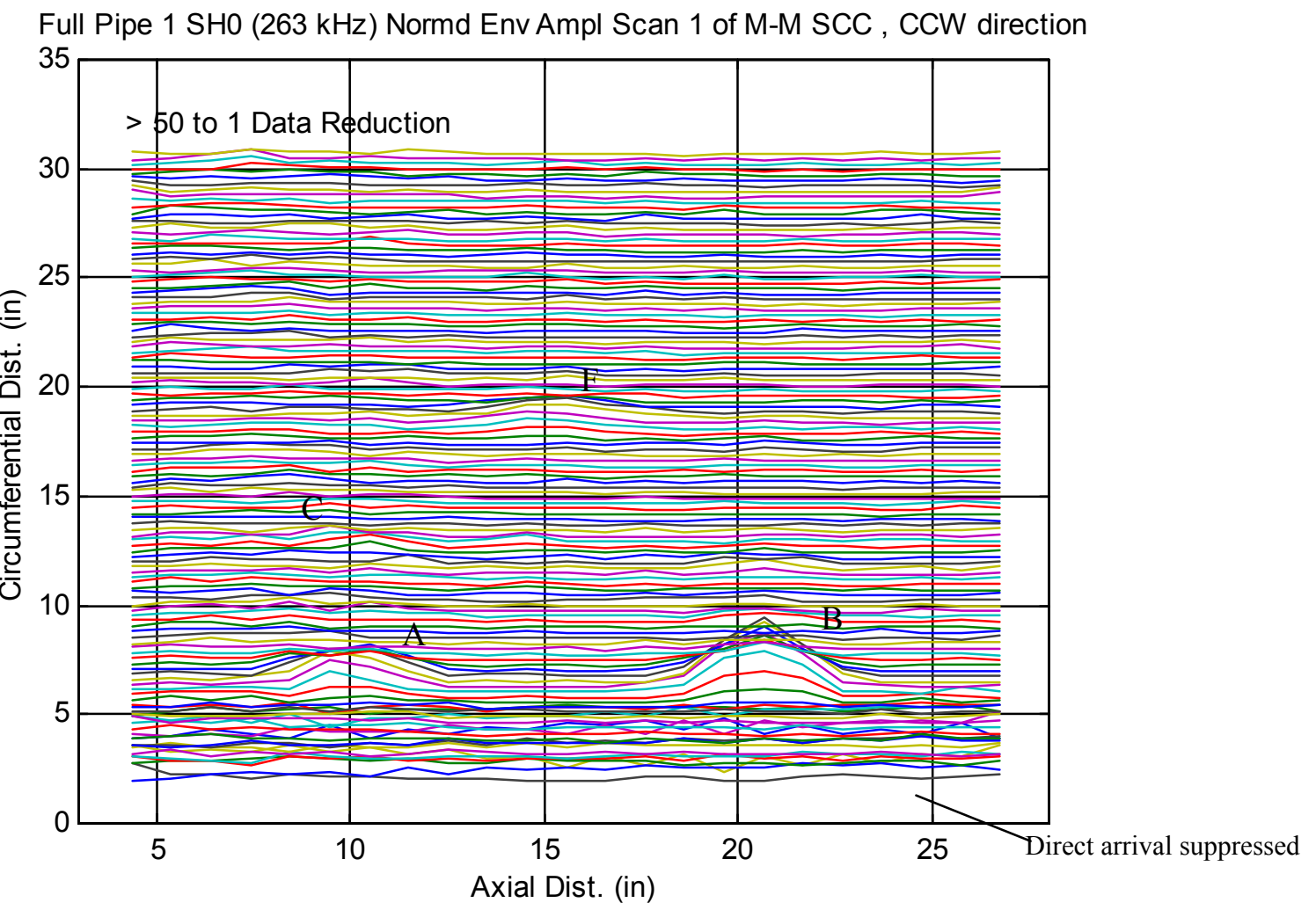

Figure 53 Channelized data corresponding to prototype mule reflection data in Figure 52, shown in A-scan. The direct arrival in Figure 53 has been suppressed.

\section{0" Full Pipe SHO Mule Component Data Analysis.}

Figures 50 and 51 show there was enough good quality signal to observe arrivals travelling around the pipe. The single revolution direct arrivals were from 0.2 to 0.3 in normalized amplitude. This was mostly due to spreading. An examination of the direct arrivals before normalization showed a variation of about $50 \%$ versus axial distance for the 23 waveforms. This was similar to the mouse measurements and was not bad.

Four of the six SCC defects, labelled A B C F in the geometry schematic (Figure 24), appeared in Figure 52 in correct places and had nearly the correct axial lengths.

The circumferential widths of the normalized reflections were much wider than the defects. If the reflection widths were determined from $70 \%$ of the peak, the widths would be about $1.5 "$ even though the cracks are .010" wide. This was because the incident wave envelope was at least four cycles (18 us) long, so reflected energy would appear at least that long. At the SHO group speed, this duration corresponds to more than 2 inches

Normalized reflection peak amplitudes of defects A and B were $30 \%$ and $55 \%$, respectively, This was what was expected for $\mathrm{SH} 0$ according to 2002 work in modelling and flat plate measurements. Basically, the work indicated that for long and relatively deep cracks 
(reflectors), the reflection is proportional to crack depth, and percentage depth approximately equals percentage reflection amplitude.

The following paragraphs discuss the remaining defects and how their reflected levels were reduced. The normalized reflection amplitudes of $C$ and $F$ were about 0.15 to 0.20 , which was low for defects of $60 \%$. Without other factors we would have expected 0.5 to 0.6 for this defect depth. However, defect $C$ was in the acoustic shadow of defect $A$, so incident and reflected wave energy were each reduced by a transmission through defect $A$. Going through defect $A$ the incident and reflected waves should each be reduced by a factor of 0.85 for a total reduction by a (multiplicative) factor of 0.7 . The reflection from $C$ would be further reduced because $C$ was only 1" long compared to an acoustic beam-width of at least 2.5 ", so not all the beam was intersected and reflected. Thus, the reflector and its reflection would be 0.4 of what they would have been if the beam were fully intersected. Taking into account the shadowing and the reduced defect length, we would have expected to see a normalized reflection of: $0.6 \times 0.7 \mathrm{x}$ $1 / 2.5=0.17$. This was about what was observed for defect $C$.

In the case of defect $\mathrm{F}$, the shadowing defect, $\mathrm{D}$, with depth of only $15 \%$, had minimal reduction effect, perhaps a total reduction by a factor of 0.9 . However, the 1" length compared to the 2.5" beam would have reduced the defect $F$ reflection, so it was only 0.4 times full value. Thus, we would have expected a value of: $0.6 \times 0.9 \times 1 / 2.5=0.22$. This was about what was observed. It is interesting to note that the calculations show that the reflection from defect $F(0.22)$ should have been greater than the reflection for $C(0.17)$. Figure 52 shows this.

Based on the previous discussion for the defects $C$ and $F$, the length and shadow effects can be determined by geometry. Then, just as we rationalized reduced reflection amplitudes, we can invert reduction factors in order to compensate shadowing and length, and thus obtain compensated defect depth estimates.

Unfortunately, defects $D$ and $E$ were missing, because they were below the noise level cut off (0.1) in Figure 52, as explained in the following discussion. Defect $D$ (15\%, 1 " long) was shallow and short, so it made a small reflection, which might have been 0.06 or less. Because defect $E(30 \%, 1 "$ long), was behind defect B (60\% depth, 3" long), E's incident and reflected energy were reduced by a factor of $0.4(1.0-6)$. It would have been further reduced by the $1 "$ length, so that defect E's expected reflection would have been on the order of 0.02 and thus in the noise. 


\section{Summary and Conclusions}

\section{Summary}

This report describes experiments and results for a project to develop a pipeline in-line inspection tool that uses electromagnetic acoustic transducers (EMATs) to detect and grade stress corrosion cracking (SCC). The time period is from October 2001 through the end of September 2004. There is a brief introduction that gives motivation, background material about EMATs and relevant previous work for the Gas Technology Institute. The previous work left six choices for the acoustic modes and a choice between magnetostrictive or Lorentz type transducers for this project.

The experimental section describes the techniques of various measurements and the systems to make them. The main purpose of these measurements and systems was to narrow the transducing choices and arrive at components for a commerial tool. The lab systems consisted at first of large, general purpose lab equipment boxes plus electro-magnet biased EMATs, then a moveable lab mouse attached to lab equipment, and then pull mule components including compact, permanent magnet biased EMATs. The mule electronic components were small and efficient and could be deployed in a commercial system. The electronics and the commercial tool design stategy were described.

Measurements were as follows: signal to noise ratios, power, insertion loss, transducer beamwidth, measurement sensitivity to tilt and standoff, dispersion due to varying wall thickness, attenuation due to external coating, and crack interactions. The crack interactions were first made on flat plate (man-made cracks) then with the mouse (SCC) in full pipe and finally with mule components in full pipe (man-made cracks).

Based on the measurements, the results section first justifies the mode choices - SHO (at 2.1 $\mathrm{MHz}-\mathrm{mm}$ ) and SV1 (at $3.9 \mathrm{MHz}-\mathrm{mm}$ ). This is because they had good SNRs and modal purity, useful crack sensitivity, and practical implementations. The other modes had distinct problems such as, a very low SNR, too high a frequency, too high a theoretical attenuation, or poor spatial resolution Because both $\mathrm{SHO}$ and SV1 were evenly matched and because they were complimentary in grading $\mathrm{cracks}^{8}$, they were both were pursued until the mule. At that time wave velocity measurements showed that SV1 was not practical to use because as wall thickness varied over a moderatel range, arrival amplitudes and timing would be distorted. Thus, SV1 was dropped.

As for the type of transduction, we decided on magnetostrictive. After achieving best magnetic biasing, similar SNRs were achieved for both magnetostrictive and Lorentz. However, magnetostrictive is more rugged than Lorentz because it allows the bias magnets to be separated from the EMAT coil. In contrast, a Lorentz EMAT, must have the bias magnet above the coil, which can place a dangerous load on the coil. Another advantage of the magnetostrictive is that the bias field is in the plane of the pipe rather than perpendicular. A perpendicular field is difficult to set up over a large area, without some spreading to planarity.

\footnotetext{
${ }^{8} \mathrm{SV} 1$, being more sensitive to shallow cracks, could be used to differentiate and grade shallow cracks, while $\mathrm{SH}$, which is disproportionally sensitive to deep cracks, could be used to identify and grade deep ones.
} 
In the Results and Conclusions section, measurements are documented by means of parametric curves - for example, reflection coefficient versus crack depth, received amplitude versus bias levels, received amplitude versus standoff.

This section shows lab mouse reflection and transmission scan measurements in 24 inch pipe for both the SHO (263 kHz or $2.1 \mathrm{MHz}-\mathrm{mm}$ ) and SV1 (470 kHz or $3.9 \mathrm{MHz}-\mathrm{mm}$ ) modes. These show that normalized reflection measurements are sensitive to and correlate with SCC. There is a performance summary for the mule prototype circuits and permanent magnet EMATs.

The section then describes effective processing - envelop extraction, digital filtering and decimation - which compresses data for storage and allows display in traditional pipeline A and $\mathrm{C}$ scans. This processing could be implemented within a commercial tool. Manually scanned waveform data from mule components illustrates (with $A$ and $C$ scan plots) this processing and responses to SCC-like cuts of known dimensions in 30 inch pipe. .

There is a detailed analysis of the use of normalized reflections from this scan to estimate crack dimensions. Axial length is clear in the $C$ Scans. It seems that depth estimates are good for cracks at least $30 \%$ deep. However, there are shadow and short defect effects. Because these effects are predicable, they may be compensated if the reflections from the defects are above the noise level.

\section{Conclusions}

Lab measurements, mouse results, and mule component results yield a number of conclusions for magnetostrictively generated SHO (at 2.1 MHz-mm ) and SV1 ( at 3.9 MHz-mm). These are given below:

Magnetostrictive EMATs were built and operated successfully. SNRs of $>100: 1$ and $\sim 30: 1$ have been achieved for SV1 and SH0, respectively with a stand-off of $\sim .1 \mathrm{~mm}$, high bias field (magnetic saturation) conditions, and a peak drive current of $\sim 10 \mathrm{~A}$ for the transmit EMAT coil using double-layer coils in series.

Measurements of reflection and transmission coefficients on a flat plate showed useful sensitivity to cracks for both the SHO and SV1 modes. Additionally, the measured coefficients agreed well with theoretic values computed by means of 2 D FEM simulations. SV1 was sensitive to shallow cracks, while SHO was sensitive to deeper (>20\% wall thickness) cracks.

Beam width measurements, measured on a plate, showed that good, non divergent beams could be launched over useful distances like about $500 \mathrm{~mm}$. This was done for an 80 by $60 \mathrm{~cm}$ EMAT that was 5 wavelengths long. In the non-divergent zone the width was about that of the EMAT coil. Measurements also indicated that the EMAT did not have sidelobes.

Standoff measurements verified that standoff decreases amplitude exponentially with the ratio of standoff to wavelength. Thus, very short wavelengths (or high frequencies) are at a disadvantage. Measurements showed that the SHO and SV1 could tolerate 1 to $2 \mathrm{~mm}$ of standoff. There was a similar tolerance for tilt.

Various lab results and checks on the group and phase velocities of launched waves confirmed the requirements for magnetic bias directions. For magnetostrictive EMATs launching waves circumferentially these requirements are: SH0 requires an axial magnetic bias, but SV1 requires 
a circumferential bias. Both of these are in the plane of the pipe wall not perpendicular to the pipe wall.

Magnetic biasing investigations were carried out on a plate, sections of pipe, and in full pipe using electro-magnets. The final results showed that for transmitter and receiver EMATs for both SH0 and SV1, the highest SNRs would be obtained with the steel with as high a B field as possble. That is at staturation.

EMAT reflection and transmission measurements made on 120 degree arc sections of dug pipe containing real SCC only showed some qualitative crack sensitivity.

A lab mouse was built and successfully deployed on real pipe with some SCC defects with two carriers, one for SH0 and one for SV1. Each mouse had a pitch-catch arrangement with electromagnet biasing.

Lab mouse data had good SNRs and showed that direct arrival amplitude varied widely along the pipe axis for both SHO and SV1. These variations were due to variations in standoff, magnetic properties affecting bias levels, and magnetostrictive properties.

In spite of variations, if the mouse reflection signals were divided by direct arrival amplitudes and so processed into normalized reflections, the normalized reflections were sensitive to and correlated with SCC. The normalization compensated for amplitude variations. This was true for both $\mathrm{SHO}$ and SV1. For SHO the sensitivity of normalized reflections to SCC was corroborated by the prototype mule scans.

Mouse transmission measurements were not very sensitive to SCC, even if normalized. Various normalizations were tried, based on the backward direct arrival but could not overcome weak defect effects on amplitude, large coupling variations, and spreading loss in the normalization.

Prototype circuits (power amp, high voltage power supplies, a preamp and filter) and permanent magnet EMATs were completed and evaluated for a mule. These are compact and of low enough power drain to use in a commercial tool. These gave good SNR and showed that operation on battery supply and with permanent magnet biasing is feasible. Special features such as firing rate random jitter effectively improved signal to noise levels.

Measurements made with mule components showed that there was no velocity dispersion for a reasonable wall thickness variation for SHO (at 2.1 MHz-mm). However, for SV1 (at $3.9 \mathrm{MHz}-$ $\mathrm{mm}$ ) dispersion could distort and spread arrivals. Because of this further investigation of SV1 was dropped.

Measurements, also done with mule components, in pipe that was externally coated showed that for $\mathrm{SHO}$ (at $2.1 \mathrm{MHz}-\mathrm{mm}$ ) there was some attenuation. It is our judgement that this attenuation is tolerable.

Simple envelope extraction processing proved to be useful for normalized reflections. This processing can be implemented within an ILI tool by means of a DSP chip. This processing results in an almost 60 to 1 reduction in storage requirements. It facilitates $A$ and $C$ scan displays like those already in use for other ILI data. It also facilitates the use of existing Tuboscope software for those displays, grading, and data handling. 
Results for a SHO scan on 30 inch pipe (having man-made axially oriented cracks) obtained by means of the prototype mule circuits and EMATs were very encouraging. In a C scan display of normalized reflections, the larger defects were detected in the correct places and had approximately the correct axial lengths. Two of the smaller defects were below the noise level. For any detected defect, width (circumferentially) was overstated because it is determined by the duration of the transmitted wave. This must be long in order to excite a pure enough guided wave with good signal to noise ratio.

Detailed analysis of the normalized reflection traces indicated that these may be treated like reflection coefficients and that for defects long enough, normalized reflection peak amplitude is nearly proportional to crack depth.

Further analysis also showed that there are crack shadow and length effects. If a crack is behind another for the circumferentially travelling waves, its reflection will be reduced. Also, if it is smaller than the beam width (e.g. the EMAT width), the peak reflections will be reduced, so depth will be under estimated. However, both of these reductions could be compensated for if the defects show up in $\mathrm{C}$ scans. Calculations also showed that such reductions would cause two defects to be below the noise level, so they would be missing in the C-scan.

Taken together the previous three paragraphs indicate that effective crack depth and axial length determination (i.e. grading) is possible when supplemented with compensation for shadow and length effects.

The first EMAT scans of SCC type defects with commercial prototype components are very encouraging. Cracks of $30 \%$ or greater of the wall thickness in depth were imaged. Their depths and lengths could be estimated from the data. These images were produced by battery powered hardware compact and rugged enough to work on an ILI tool. Based on results with mule components, average power and acquisition requirements are feasible at reasonable logging speeds. In sum, nothing seen so far is a large hurdle for a full sized tool.

The next steps in this project would be to fully implement the diagram of Figure 20 with mule prototype circuits and a suitable recorder plus ADCs. This should be pulled at first at moderate speeds in test pipes with man-made cracks and real SCC. At that time, we should use the results to address issues such as: compensation details for length, shadowing, and grading, responses to real SCC families, motion-induced noise, and EMAT sensor head protection. Next or even in parallel, the pulled device should be turned into a commercial prototype and then run in a gas line. 


\section{References}

1. T. A. Bubenik, D.R. Stephens, et al, Stress Corrosion Cracks in Pipelines: Characteristics and Detection Considerations, Gas Research Institute Topical Report (for GRI Contract 5093-220-2692), April 1995.

2. H. M. Frost, "Electromagnetic-Ultrasound Transducers: Principles, practice, and applications", Physical Acoustics Vol. XIV (Academic Press, 1979), pp 179-275. .

3. B. W. Maxfield and C.M. Fortunko, The Design and Use of Electromagnetic Acoustic Wave Transducers (EMATs), Materials Evaluation, 41, 1399-408, (1983).

4. L W. Schmerr, Fundamentals of Ultrasonic NonDestructive Evaluation, A Modelling Approach, Plenum, Press 1998.

5. J. L. Rose, Ultrasonic Waves in Solid Media, Cambridge University Press, 1999.

6. R. Bruce Thompson, Generation of horizontally polarized shear waves in ferromagnetic materials using magnetostrictively coupled meander-coil electromagnetic transducers, Applied Physics Letters Vol. 15, pp 175-177, January 1979.

7. Riichi Murayama, Driving mechanism on magnetostrictive type electromagnetic acoustic transducer for symmetrical vertical-mode Lamb wave of shear horizontal-mode plate wave, Ultrasonics Vol 34 ,1996, Elsevier Publishing, pp 729-736.

8. R. B. Thompson, "Measurements with EMAT Transducers", Physical Acoustics Vol. XIX, Academic Press, pp 164-200, 1990.

9. M. G. Silk and K. F. Bainton, The propagation in metal tubing of ultrasonic wave modes equivalent to Lamb waves, Ultrasonics, 1979: pp 11-19.

10. J. L. Rose and X. Zhao, Anomaly Throughwall Depth Measurements With Shear Guided Waves, Materials Evaluation, October 2001, pp 1234-1238.

11. J C Hamilton, The Development of An EMAT Based In-Line Inspection System For the Detection of Stress Corrosion Cracks In Operating Pipelines, Gas Research Institute Final Report (for Contract 5095-270-3286), April 2000.

12. J. Aron, J Gore, et al, Advanced Electro Magnetic Acoustic Transducer (EMAT) Technology for Determining Stress Corrosion Cracking (SCC), Gas Research Institute Topical Report (for GRI Contract 6030), January 2002.

13. J. Aron, J Gore, et al, Development of an EMAT In-Line Inspection System For Detection, Discrimination, and Grading of Stress Corrosion Cracking In Pipelines), DOE Annual Technical Report for 2002, (DOE Award \# DE-FC26-01NT41154), July 2003

14. J. Aron, J Gore, et al, Development of an EMAT In-Line Inspection System For Detection, Discrimination, and Grading of Stress Corrosion Cracking In Pipelines), DOE Annual Technical Report for 2003, (DOE Award \# DE-FC26-01NT41154), April 2004 